\title{
CLASSIFICAÇÃO E TOPOLOGIA DE VARIEDADES SINGULARES PARAMETRIZADAS POR MULTIGERMES
}

\author{
Roberta Godoi Wik Atique
}

Orientador:

Prof. Dr. David Mond

Co-orientadora:

Profa. Dra. Maria Aparecida Soares Ruas

Tese apresentada ao Instituto de Ciências Matemáticas de São Carlos da Universidade de São Paulo, como parte dos requisitos necessários para obtenção do título de Doutor em Ciências - Área: Matemática.

USP - São Carlos

Março - 1998 
A Mariana 
Agradeço a tí, ó Deus, pelas bençãos que tens derramado sobre nossas vidas, em especial pela conclusão deste trabalho.

Por que estás abatida, ó minha alma?

Por que te perturbas dentro de mim?

Espera em Deus, pois ainda o louvarei, a ele, meu auxilio e Deus meu.

Salmo 42:5.

Grandes cousas fez o Senhor por nós; por isso, estamos alegres.

Salmo 126:3. 


\section{AGRADECIMENTOS}

Ao Evandro, o meu agradecimento todo especial pelo apoio sempre presente, por ter me suportado quando estava insuportável, por ter deixado tudo aqui e me acompanhado até a Inglaterra.

Aos meus pais, com muito carinho, por tudo que sempre fizeram por mim.

Tive a honra de trabalhar com dois nomes importantes em Teoria de Singularidades. Mais do que isto, encontrei dois amigos que me ensinaram, incentivaram e colaboraram decisivamente para a realização deste trabalho. Aos meus orientadores David Mond e Maria Aparecida Soares Ruas meu mais sincero agradecimento.

À Miriam e Camilo pela acolhida em Coventry.

Aos colegas do Departamento de Matemática do ICMSC-USP.

Ao Mathematics Institute da Universidade de Warwick, Inglaterra.

À CAPES. 


\section{RESUMO}

Neste trabalho obtemos a classificação dos multigermes simples de $\mathbb{C}^{2}$ em $\mathbb{C}^{3}$ bem como resultados sobre sua topologia. Para estes multigermes mostramos que o complementar do conjunto de bifurcação é um espaço de Eilenberg-MacLane. Para alguns multigermes de $\mathbb{C}^{n}$ em $\mathbb{C}^{p}$ de codimensão 1 e coposto 1 , mostramos que sua forma real admite uma perturbação real cujo discriminante tem o mesmo tipo de homotopia que seu correspondente complexo.

\section{ABSTRACT}

In this work we obtain the classification of simple multigerms of maps from 2-space to 3-space and also some results about their topology. For some codimension 1 and corank 1 multigerms from $\mathbb{C}^{n}$ to $\mathbb{C}^{p}$, we show that we can find a real form and a real perturbation such that the inclusion of the real discriminant in that of the complexification induces an isomorphism on the vanishing homology. 


\section{Sumário}

1 Introdução 1

2 Classificação de multigermes simples de $\mathbb{C}^{2}$ em $\mathbb{C}^{3}$

2.1 Introdução . . . . . . . . . . . . . . . . 5

2.2 Resultados Gerais . . . . . . . . . . . . . . . . . . . 7

2.3 Classificação de bigermes . . . . . . . . . . . . . . 18

2.4 Classificação de trigermes $\ldots \ldots \ldots \ldots \ldots$. . . . . . 27

2.5 Adjacências . . . . . . . . . . . . . . . 35

2.5.1 Adjacências de bigermes . . . . . . . . . . . 35

2.5 .2 Adjacências de trigermes . . . . . . . . . . . . 36

3 A topologia do complementar do conjunto de bifurcação $\quad 37$ 3.1 Introdução . . . . . . . . . . . . . . . 37

3.2 O conjunto de bifurcação . . . . . . . . . . . . . . 38

4 A topologia da imagem de uma perturbação estável 45

4.1 Introdução . . . . . . . . . . . . . . . . 45

4.2 Método Goryunov-Mond . . . . . . . . . . . . . . . 46

4.3 O número de Milnor da imagem de certos monogermes e trigermes . . . . . . . . . . . . . . . 50 


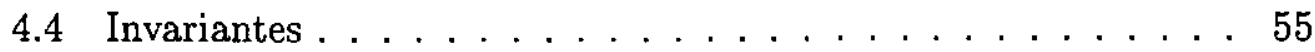

4.4 .1 Bigermes . . . . . . . . . . . . . 56

4.4 .2 Trigermes $\ldots \ldots \ldots \ldots \ldots \ldots$

5 Multigermes de $\mathcal{A}_{e}$-codimensão 1

5.1 Introdução . . . . . . . . . . . . . . . 61

5.2 Aumentação . . . . . . . . . . . . . . . . . 62 62

5.3 Classificação de alguns multigermes de $\mathcal{A}_{e}$-codimensão $1 \ldots 70$

5.4 Topologia . . . . . . . . . . . . . . . . 86

6 Multigermes de $\mathcal{A}_{e}$-codimensão arbitrária 98

6.1 Introdução . . . . . . . . . . . . . . . . 998

6.2 Resultados gerais . . . . . . . . . . . . . . . 99

6.3 Topologia da aumentação $\ldots \ldots \ldots 100$ 


\section{Capítulo 1}

\section{Introdução}

Classificar germes e encontrar formas normais é um problema clássico em teoria de singularidades. O conhecimento das formas normais permite estudar o comportamento de tais germes, em especial, sua topologia.

Uma outra questão é encontrar formas reais para germes complexos e para suas perturbações de modo que o discriminante real tenha o mesmo tipo de homotopia que seu correspondente complexo.

Este trabalho tem por objetivo explorar os conceitos acima para multigermes. Assim, obtemos a classificação dos multigermes simples de $\mathbb{C}^{2}$ em $\mathbb{C}^{3}$ bem como resultados sobre sua topologia. Para estes multigermes mostramos que o complementar do conjunto de bifurcaçāo é um espaço de Eilenberg-MacLane, $K(\pi, 1)$. Para multigermes de $\mathbb{C}^{n}$ em $\mathbb{C}^{p}, n \geq p$, de $\mathcal{A}_{e}$-codimensão 1 e coposto 1 , mostramos que as mudanças na topologia do discriminante complexo resultante de uma deformação do germe, podem ser observadas no correspondente real.

A classificação dos multigermes simples de $\mathbb{C}^{2}$ em $\mathbb{C}^{3}$, que é obtida no Capítulo 2, completa a classificaçāo dos germes simples iniciada por D.Mond. 
Em [26] Mond classifica os monogermes simples de $\mathbb{R}^{2}$ em $\mathbb{R}^{3}$ e obtem resultados parciais para bigermes. Em particular, Mond dá a lista dos monogermes simples, que vale também para o caso complexo.

Nosso método de classificação consiste em mostrar que a $\mathcal{A}$-classificação de multigermes de $\mathbb{C}^{n}$ em $\mathbb{C}^{n+1}$ se reduz à classificação de germes de funções definidas em $\mathbb{C}^{n+1}$, segundo o subgrupo $\mathcal{K}_{V}$ do grupo de contato $\mathcal{K}$.

No caso dos trigermes mostramos que existe uma forma normal dada por germes de funções numa variedade com bordo. Assim, a $\mathcal{A}$-classificação de tais trigermes é equivalente à classificação (devida a V.I.Arnold) de germes de funções numa variedade com bordo com respeito a um subgrupo do grupo $\mathcal{K}$. Este fato é importante visto que alguns monogermes são obtidos destas mesmas funções.

No Capítulo 3 mostramos que o complementar do conjunto de bifurcação de um multigerme simples de $\mathbb{C}^{2}$ em $\mathbb{C}^{3}$ é um espaço de Eilenberg-MacLane $K(\pi, 1)$. Este resultado foi obtido por V.V.Goryunov em [3] para os monogermes simples.

Dado um multigerme $f$ de $\mathbb{C}^{n}$ em $\mathbb{C}^{n+1}$, finitamente determinado, $(n, n+$ 1) no domínio das boas dimensōes segundo Mather, podemos construir uma fibração definida na imagem de um desdobramento versal de $f$ com valores no complementar do conjunto de bifurcação. A fibra tem o tipo de homotopia de um bouquet de n-esferas. O número de esferas no bouquet é chamado número de Milnor da imagem de $f$.

Os monogermes da forma $\left(x, y^{2}, y p\left(x, y^{2}\right)\right)$ e os trigermes simples possuem o mesmo conjunto de bifurcação, a saber o discriminante da correspondente função do bordo. Logo surge a questão: qual a relação entre as fibraçōes acima descritas? No Capítulo 4 mostramos que as fibras são homotópicas. Para isto usamos o método desenvolvido por D.Mond e V.V.Goryunov, que 
utiliza sequências espectrais, para calcular o número de Milnor da imagem de um germe.

Os resultados acima descritos estão reunidos no artigo On the classification of multigerms of maps from $\mathbb{C}^{2}$ to $\mathbb{C}^{3}$ under $\mathcal{A}$-equivalence, que foi submetido para publicação no Quartely Journal of Mathematics, da Universidade de Oxford, Inglaterra.

Em sua tese de doutorado na Universidade de Warwick, T.Cooper classifi ca multigermes de $\mathbb{C}^{n}$ em $\mathbb{C}^{n+1}$ de coposto 1 e $\mathcal{A}_{e}$-codimensão 1 . Ele também prova que tais multigermes têm número de Milnor da imagem igual a 1 e possuem formas reais com boas perturbações reais. Resultados análogos foram obtidos por D.Mond para monogermes de $\mathbb{C}^{n}$ em $\mathbb{C}^{p}, n \geq p$, de coposto 1 e $\mathcal{A}_{e^{-}}$ codimensão 1 , substituindo imagem por discriminante. O método de Cooper consiste na aumentação, que é um processo através do qual se obtem novos multigermes de $\mathcal{A}_{e}$-codimensão 1 a partir de multigermes também de $\mathcal{A}_{e^{-}}$ codimensão 1. Ele obtem uma classificação de multigermes primitivos (não aumentados) de $\mathbb{C}^{n}$ em $\mathbb{C}^{p}$ e mostra que alguns resultados sobre a topologia de germes podem ser estendidos para suas aumentações. No Capítulo 5 descrevemos as técnicas acima, que são utilizadas para mostrar que multigermes de $\mathbb{C}^{n}$ em $\mathbb{C}^{p}, n \geq p$, de coposto 1 e $\mathcal{A}_{e}$-codimensão 1 são quasihomogêneos, logo têm número de Milnor do discriminante igual a 1, e possuem formas reais com boas perturbações reais. Estes resultados reforçam a conjectura de que todas as singularidades de codimensäo 1 possuem formas reais com boas perturbações reais, e estão reunidos no preprint Vanishing topology of codimension 1 multigerms over $\mathbb{R}$ and $\mathbb{C}$.

Um resultado central do Capítulo 5 mostra que o discriminante de uma perturbação estável da aumentação é a suspensão do discriminante de uma perturbação estável do germe. No Capítulo 6 definimos aumentação para 
multigermes de codimensão arbitrária e mostramos um resultado análogo ao acima descrito. 


\section{Capítulo 2}

\section{Classificação de multigermes simples de $\mathbb{C}^{2}$ em $\mathbb{C}^{3}$}

\subsection{Introdução}

Nosso objetivo neste capítulo é obter a lista completa dos multigermes simples de $\mathbb{C}^{2}$ em $\mathbb{C}^{3}$.

A lista dos monogermes simples de $\mathbb{C}^{2}$ em $\mathbb{C}^{3}$ obtida por D.Mond em [26] é a seguinte:

$$
\begin{array}{lcc}
\text { Monogerme } & \mathcal{A}_{e}-\text { codim } & \text { Nome } \\
f(x, y)=(x, y, 0) & 0 & \text { Imersão } \\
f(x, y)=\left(x, y^{2}, x y\right) & 0 & \text { Cross }- \text { cap }\left(S_{0}\right)
\end{array}
$$




$$
\begin{array}{llll}
f(x, y)=\left(x, y^{2}, y^{3}+x^{k+1} y\right), & k \geq 1 & k & S_{k} \\
f(x, y)=\left(x, y^{2}, x^{2} y+y^{2 k+1}\right), & k \geq 2 & k & B_{k} \\
f(x, y)=\left(x, y^{2}, x y^{3}+x^{k} y\right), \quad k \geq 3 & k & C_{k} \\
f(x, y)=\left(x, y^{2}, x^{3} y+y^{5}\right) & & 4 & F_{4} \\
f(x, y)=\left(x, y^{3}, x y+y^{3 k-1}\right), \quad k \geq 2 & k & H_{k}
\end{array}
$$

Existem 5 multigermes de $\mathbb{C}^{2}$ em $\mathbb{C}^{3}$ de $\mathcal{A}_{e}$-codimensão 1, que são:

1. $S_{1}$.

2. Contato não transversal de duas folhas imersas.

3. Intersecção de cross-cap e plano imerso.

4. Intersecção quadrupla.

5. Intersecção de 3 folhas imersas, duas a duas transversais onde cada folha tem contato do tipo $A_{1}$ com a intersecçāo das outras duas.

Em [18], C.Hobbs e N.Kirk obtem a lista de multigermes simples de $\mathbb{R}^{2}$ em $\mathbb{R}^{3}$ usando um método diferente. 


\section{$2.2 \quad$ Resultados Gerais}

Seja $S$ um subconjunto de $\mathbb{C}^{n}$. Consideremos o conjunto das aplicações analíticas $U \rightarrow \mathbb{C}^{p}$ cujo domínio $U$ é uma vizinhança de $S$ em $\mathbb{C}^{n}$. Neste conjunto introduzimos a seguinte relação de equivalência: duas aplicações $f: U \rightarrow \mathbb{C}^{p}$ e $g: V \rightarrow \mathbb{C}^{p}$ são equivalentes se existir uma vizinhança $W$ de $S$ em $\mathbb{C}^{n}$ tal que $\left.f\right|_{W}=\left.g\right|_{W}$. As classes de equivalência são chamadas multigermes de $f$ em $S$ e denotadas por $f:\left(\mathbb{C}^{n}, S\right) \rightarrow\left(\mathbb{C}^{p}, f(S)\right)$. Quando $S$ consiste de um único elemento tais classes também são chamadas de monogermes. Usaremos o termo germe de uma maneira mais geral, podendo significar um monogerme ou multigerme.

Consideraremos $S$ um conjunto finito, ou seja, $S=\left\{z_{1}, \ldots, z_{r}\right\}$. É suficiente estudar multigermes onde $f(S)=\{0\}$, uma vez que a classificação de multigermes com $f(S)$ finito e não unitário se reduz a este caso. O germe de $f$ em $z_{i}$ será chamado de ramo e denotado por $f^{(i)}:\left(\mathbb{C}^{n}, z_{i}\right) \rightarrow\left(\mathbb{C}^{p}, 0\right)$.

Dois multigermes $f, g:\left(\mathbb{C}^{n}, S\right) \rightarrow\left(\mathbb{C}^{p}, 0\right)$ são ditos $\mathcal{A}$-equivalentes se existirem germes de difeomorfismos $\varphi^{(i)}:\left(\mathbb{C}^{n}, z_{i}\right) \rightarrow\left(\mathbb{C}^{n}, z_{i}\right)$ e $\psi:\left(\mathbb{C}^{p}, 0\right) \rightarrow$ $\left(\mathbb{C}^{p}, 0\right)$ tais que $f^{(i)} \circ \varphi^{(i)}=\psi \circ g^{(i)}, i=1, \ldots, r$. Isto significa que para cada ramo podemos escolher uma mudança de coordenada na fonte enquanto que na meta a mesma mudança de coordenada deve ser aplicada para todos.

Um multigerme $f:\left(\mathbb{C}^{n}, S\right) \rightarrow\left(\mathbb{C}^{p}, 0\right)$ é dito $\mathcal{A}$-simples se existe um número finito de classes de $\mathcal{A}$-equivalência tal que se $f$ pertence a uma família $F:\left(\mathbb{C}^{n} \times \mathbb{C}^{d}, S \times\left\{\lambda_{0}\right\}\right) \rightarrow\left(\mathbb{C}^{p} \times \mathbb{C}^{d}, 0\right), F(x, \lambda)=\left(f_{\lambda}(x), \lambda\right), f_{\lambda_{0}}=f$, então para qualquer $\left(w_{1}, \ldots, w_{r}, \lambda\right)$ numa vizinhança suficientemente pequena de $S \times\left\{\lambda_{0}\right\}$, o multigerme de $f_{\lambda}$ em $\left\{w_{1}, \ldots, w_{r}\right\}$ pertence a uma destas classes de equivalência.

Seja $\mathcal{O}_{n}$ o anel local dos germes de funções analíticas $f:\left(\mathbb{C}^{n}, 0\right) \rightarrow \mathbb{C}$ e 
$\mathcal{M}_{n}$ seu ideal maximal $\left(f \in \mathcal{M}_{n}\right.$ se $f(0)=0$ ). O conjunto dos monogermes $f:\left(\mathbb{C}^{n}, 0\right) \rightarrow \mathbb{C}^{p}$, denotado por $\mathcal{O}(n, p)$, é um $\mathcal{O}_{n}$-módulo livre de posto $p$. O submódulo consistindo dos germes que satisfazem $f(0)=0$ é dado por $\mathcal{M}_{n} \cdot \mathcal{O}(n, p)$. Assim o conjunto dos multigermes $f:\left(\mathbb{C}^{n}, S\right) \rightarrow\left(\mathbb{C}^{p}, 0\right)$ é isomorfo à soma direta de $r$ cópias de $\mathcal{M}_{n} \cdot \mathcal{O}(n, p)$.

O espaço tangente a $f:\left(\mathbb{C}^{n}, S\right) \rightarrow\left(\mathbb{C}^{p}, 0\right)$ segundo o grupo $\mathcal{A}$ é dado por (ver C.T.C.Wall [35]):

$$
T \mathcal{A} f=t f\left(\mathcal{M}_{n} \theta(n)_{S}\right)+\omega f\left(\mathcal{M}_{p} \theta(p)\right) \subseteq \mathcal{M}_{n} \theta(f)
$$

onde $\theta(n)$ consiste dos germes em 0 de campos vetoriais em $\mathbb{C}^{n}, \theta(n)_{S}$ é a soma direta de $r$-cópias de $\theta(n)$ e $\theta(f)$ consiste dos germes em 0 de campos vetoriais ao longo de $f$. Neste caso $\theta(f)$ é a soma direta de $\theta\left(f^{(i)}\right)$, onde $f^{(i)}$ é ramo de $f$, e pode ser identificado com a soma direta de $r$-cópias de $\mathcal{O}(n, p)$. O espaço tangente estendido é dado por

$$
T \mathcal{A}_{e} f=t f\left(\theta(n)_{s}\right)+\omega f(\theta(p)) \subseteq \theta(f)
$$

e a $\mathcal{A}_{e}$-codimensão de $f$ é dada por

$$
\operatorname{dim}_{\mathbb{C}} \frac{\theta(f)}{T \mathcal{A}_{e} f}=\operatorname{dim}_{\mathbb{C}} N \mathcal{A}_{e} f
$$

onde $N \mathcal{A}_{e} f=\frac{\theta(f)}{T \mathcal{A}_{e} f}$ é chamado de espaço normal a $f$.

Seja $\mathcal{R}$ o grupo dos germes de difeomorfismos $\psi:\left(\mathbb{C}^{p}, 0\right) \rightarrow\left(\mathbb{C}^{p}, 0\right)$.

Consideremos $(\mathcal{X}, 0)$ um germe de um espaço analítico em $\left(\mathbb{C}^{p}, 0\right)$ e seja $I \subseteq \mathcal{O}_{p}$ um ideal que define $\mathcal{X}$, ou seja, o ideal dos germes de funções que se anulam em $\mathcal{X}$. Denotamos por $\mathcal{R}_{\mathcal{X}}$ o subgrupo de $\mathcal{R}$ consistindo dos germes $\psi \in \mathcal{R}$ tais que $\psi^{*}(I)=I$, ou seja, $\psi(\mathcal{X})=\mathcal{X}$. Este grupo tem sido estudado por vários autores. As principais referências são: W.Bruce and M.Roberts [9], J.Damon [12], S.Izumiya and S.Matsuoka [19] e R.Pellikaan [31]. 
Definimos $\mathcal{K}$ (grupo de contato) como o grupo dos germes de difeomorfismos $H:\left(\mathbb{C}^{p} \times \mathbb{C}, 0\right) \rightarrow\left(\mathbb{C}^{p} \times \mathbb{C}, 0\right), H(y, t)=(\psi(y), \tilde{H}(y, t))$ onde $\psi \in \mathcal{R}$ e $\tilde{H}(y, 0)=0$. Este grupo age em $\mathcal{O}_{p}$ da seguinte maneira: se $h_{1}, h_{2} \in \mathcal{O}_{p}, h_{1}$ e $h_{2}$ são $\mathcal{K}$-equivalentes se existir $H \in \mathcal{K}$ tal que $H\left(y, h_{1}(y)\right)=\left(\psi(y), h_{2}(\psi(y))\right)$, em outras palavras $H$ leva o gráfico de $h_{1}$ no gráfico de $h_{2}$. Podemos também definir o grupo $\mathcal{K}$ como sendo o produto semi-direto de $\mathcal{C}$ e $\mathcal{R}$ (onde $\mathcal{C}$ é o grupo dos germes de difeomorfismos $H$ como acima onde $\psi$ é a identidade). Ou seja $\mathcal{K}=\mathcal{R} \cdot \mathcal{C}$. Denotamos por $\mathcal{K}_{\mathcal{X}}$ o subgrupo de $\mathcal{K}$ dado por $\mathcal{K}_{\mathcal{X}}=\mathcal{R}_{\mathcal{X}} \cdot \mathcal{C}$, em outras palavras $\mathcal{K}_{\mathcal{X}}$ é o grupo dos germes de difeomorfismos $H$ como acima onde $\psi(\mathcal{X})=\mathcal{X}$.

Seja $\mathcal{R}^{(k)}=\left\{\psi \in \mathcal{R} / \psi=i d\left(\bmod \mathcal{M}_{p}^{k+1}\right)\right\}$ e $\mathcal{R}_{\mathcal{X}}^{(k)}=\mathcal{R}_{\mathcal{X}} \cap \mathcal{R}^{(k)} . \mathrm{O}$ conjunto dos $k$-jatos de elementos de $\mathcal{R}_{\mathcal{X}}$ é dado por $J^{k} \mathcal{R}_{\mathcal{X}}=\mathcal{R}_{\mathcal{X}} / \mathcal{R}_{\mathcal{X}}^{(k)}$. Segundo R.Pellikaan [31], $J^{k} \mathcal{R}_{\mathcal{X}}$ é um grupo de Lie que age algebricamente na variedade diferenciável $J^{k}(p, 1)$. Definimos $J^{k} \mathcal{K}_{\mathcal{X}}$ analogamente.

$\mathrm{O}$ resultado seguinte reduz a $\mathcal{A}$-classificação de multigermes para a $\mathcal{K}_{\mathcal{X}^{-}}$ classificação de germes de funções.

Teorema 2.2.1 Dados $\tilde{S}=\left\{z_{1}, \ldots, z_{r-1}\right\}$ e $S=\tilde{S} \cup\left\{z_{r}\right\}$ subconjuntos de $\mathbb{C}^{n}$ e $f:\left(\mathbb{C}^{n}, \tilde{S}\right) \rightarrow\left(\mathbb{C}^{n+1}, 0\right)$ um multigerme finitamente $\mathcal{A}$-determinado, consideremos $g, \tilde{g}:\left(\mathbb{C}^{n}, S\right) \rightarrow\left(\mathbb{C}^{n+1}, 0\right)$ multigermes finitamente $\mathcal{A}$-determinados tais que $g^{(i)}=\tilde{g}^{(i)}=f^{(i)}$ para $i=1, \ldots, r-1$. Então $g$ e $\tilde{g}$ são $\mathcal{A}$-equivalentes se, e somente se, $h$ e $\tilde{h}$ são $\mathcal{K}_{\mathcal{X}}$-equivalentes onde $h, \tilde{h} \in \mathcal{O}_{n+1}$ são equações reduzidas que definem as imagens de $g^{(r)}$ e $\tilde{g}^{(r)}$ respectivamente e $\mathcal{X}$ é a imagem de $f$.

Prova Seja $\mathcal{R}_{\tau}$ o grupo dos germes de difeomorfismos $\varphi:\left(\mathbb{C}^{n}, z_{r}\right) \rightarrow$ $\left(\mathbb{C}^{n}, z_{r}\right)$. 
Então $g$ é $\mathcal{A}$-equivalente a $\tilde{g}$ se, e somente se, $g^{(r)}$ é $\left(\mathcal{R}_{r} \times \mathcal{R}_{\mathcal{X}}\right)$-equivalente a $\tilde{g}^{(r)}$.

Se $g^{(r)}$ é $\left(\mathcal{R}_{r} \times \mathcal{R}_{\mathcal{X}}\right)$-equivalente a $\tilde{g}^{(r)}$ então existem $\psi \in \mathcal{R}_{\mathcal{X}}$ e $\varphi \in \mathcal{R}_{r}$ tais que $\psi \circ g^{(r)}=\tilde{g}^{(r)} \circ \varphi$. Portanto $(\tilde{h} \circ \psi)^{-1}(0)=h^{-1}(0)$. Segundo C.Gibson [14, p.145], $\tilde{h} \circ \psi$ é $\mathcal{C}$-equivalente a $h$. Como $\psi \in \mathcal{R}_{\mathcal{X}}$, temos que $\tilde{h}$ é $\mathcal{K}_{\mathcal{X}}$-equivalente a $h$.

Reciprocamente, $\tilde{h} \mathcal{K}_{\mathcal{X}}$-equivalente a $h$ implica que existe $\phi \in \mathcal{R}_{\mathcal{X}}$ tal que $h \circ \phi$ é $\mathcal{C}$-equivalente a $\tilde{h}$. Assim, $\left(\phi^{-1} \circ g^{(r)}\right)\left(\mathbb{C}^{n}\right)=\tilde{g}^{(r)}\left(\mathbb{C}^{n}\right)$. Segue da unicidade da normalisação [24, Theorem 4.23] que $\phi^{-1} \circ g^{(r)}$ é $\mathcal{R}_{r}$-equivalente a $\tilde{g}^{(r)}$. Portanto $g^{(r)}$ é $\left(\mathcal{R}_{r} \times \mathcal{R}_{\mathcal{X}}\right)$-equivalente a $\tilde{g}^{(r)}$.

Para a classificação de multigermes, usaremos a técnica infinitesimal desenvolvida por J.Mather [23, Lemma 3.1].

Proposição 2.2.2 (Lema de Mather) Seja $G$ um grupo de Lie que age numa variedade diferenciável $M$, e seja $S$ uma subvariedade de $M$ que satisfaz as seguintes propriedades:

(1) Para qualquer $x \in S, T_{x} G \cdot x \supseteq T_{x} S$;

(2) $A$ dimensão de $G \cdot x$ independe de $x \in S$;

(3) $S$ é conexa.

Então $S$ está contida numa única G-órbita.

Nosso objetivo é determinar as $\mathcal{K}_{\mathcal{X}}$-órbitas em $\mathcal{O}_{p}$ usando o resultado acima. Para isto precisaremos do $\mathcal{K}_{\mathcal{X}}$-espaço tangente e de um critério para $\mathcal{K}_{\mathcal{X}}$-determinação finita, ver [9] e [19]. 
Lema 2.2.3 Seja $h \in \mathcal{O}_{p}$. Então

$$
\begin{gathered}
T \mathcal{R}_{\mathcal{X}, e} h=\{\xi \cdot h / \xi \in \operatorname{Der}(\log \mathcal{X})\}=\operatorname{th}(\operatorname{Der}(\log \mathcal{X})) \\
T \mathcal{R}_{\mathcal{X}} h=\left\{\xi \cdot h / \xi \in \mathcal{M}_{p} \theta(p) \cap \operatorname{Der}(\log \mathcal{X})\right\}
\end{gathered}
$$

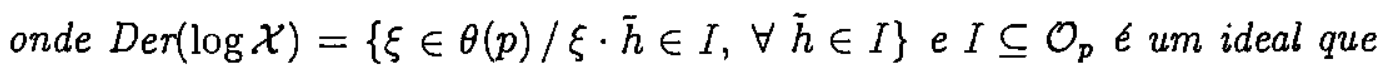
define $\mathcal{X}$.

Usaremos o programa Macaulay [8] para calcular $\operatorname{Der}(\log \mathcal{X})$ onde necessário.

O resultado seguinte é análogo ao que J.Bruce e M.Roberts obtem em [9]. Proposição 2.2.4 Se $\mathcal{M}_{p}^{k+1} \subset \mathcal{M}_{p} T \mathcal{R}_{\mathcal{X}} h+\mathcal{M}_{p}^{k+2}$ então $h$ é $k-\mathcal{R}_{\mathcal{X}}$ determinado.

Prova A prova é análoga ao caso $\mathcal{R}$, ver C.Gibson [14, p.117].

Consideremos $\tilde{h} \in \mathcal{O}_{p}$ tal que $j^{k} h(0)=j^{k} \tilde{h}(0)$. Temos de mostrar que $\tilde{h}$

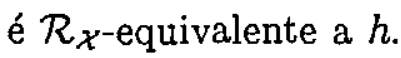

Como $\mathcal{O}_{p}$ é um espaço vetorial, podemos ligar $h$ e $\tilde{h}$ por uma reta:

$$
h_{t}(y)=H(y, t)=h(y)+t(\bar{h}(y)-h(y)) \quad t \in[0,1]
$$

$h_{t}$ é uma família a 1-parâmetro de germes com $h_{0}=h$ e $h_{1}=\tilde{h}$. Mostraremos que quaisquer dois germes desta família são $\mathcal{R}_{\mathcal{X}}$-equivalentes.

Seja $\hat{h}=\tilde{h}-h \in \mathcal{M}_{p}^{k+1}$. Então $h_{t}(y)=h(y)+t \hat{h}(y)$.

Se existir $\phi(y, t)=\phi_{t}(y)=\left(\phi_{t, 1}(y), \ldots, \phi_{t, p}(y)\right)$ tal que $h_{t} \circ \phi_{t}=h$ então a proposição segue.

Se $h_{t} \circ \phi_{t}=h$ então $\frac{d}{d t}\left(h_{t} \circ \phi_{t}\right)=\frac{d}{d t} h=0$. Portanto

$$
\sum_{i=1}^{p} \frac{\partial h}{\partial y_{i}}\left(\phi_{t}(y)\right) \cdot \frac{d \phi_{t, i}}{d t}(y)+\sum_{i=1}^{p} t \frac{\partial \hat{h}}{\partial y_{i}}\left(\phi_{t}(y)\right) \cdot \frac{d \phi_{t, i}}{d t}(y)+\left(\hat{h} \circ \phi_{t}\right)(y)=0
$$


Seja $u=\phi_{t}(y)$ e $\xi(y, t)=\xi_{t}(y)=\left(\xi_{t, 1}(y), \ldots, \xi_{t, p}(y)\right)$ tal que $\xi_{t}\left(\phi_{t}(y)\right)=$ $\frac{d \phi_{t}}{d t}(y)$. Portanto

$$
\begin{gathered}
\sum_{i=1}^{p}\left(\frac{\partial h}{\partial y_{i}}(u) \cdot \xi_{t, i}(u)+t \frac{\partial \hat{h}}{\partial y_{i}}(u) \cdot \xi_{t, i}(u)\right)=-\hat{h}(u) \\
\sum_{i=1}^{p}\left(\frac{\partial h}{\partial y_{i}}+t \frac{\partial \hat{h}}{\partial y_{i}}\right) \cdot \xi_{t, i}=-\hat{h} \\
\sum_{i=1}^{p} \frac{\partial H}{\partial y_{i}} \cdot \xi_{t, i}=-\hat{h}
\end{gathered}
$$

Se existir $\xi$, consideremos $\xi$ como um campo vetorial que depende de $t \mathrm{e}$ portanto $\phi_{t}$ é o fluxo (com a condição inicial $\phi_{0}=i d$ ). Se $\xi_{t} \in \operatorname{Der}(\log \mathcal{X})$ então $\phi_{t} \in \mathcal{R}_{\mathcal{X}}$ para $t$ suficientemente pequeno (ver Pellikaan [31, Remark 2.17]. Assim, para $t$ suficientemente pequeno, $h_{t}$ é $\mathcal{R}_{\mathcal{X}}$-equivalente a $h$. Mas podemos provar este fato para qualquer $t_{0} \in \mathbb{C}$, ou seja, para todo $s \in \mathbb{C}$

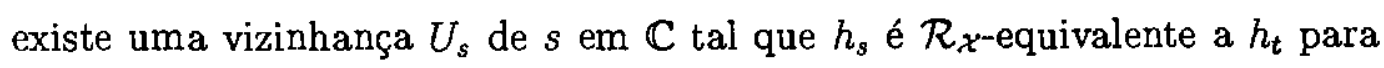
todo $t \in U_{s}$. Como 0 e 1 podem ser ligados por uma reta em $\mathbb{C}$, podemos concluir que $\tilde{h}=h_{1}$ está na $\mathcal{R}_{\mathcal{X}}$-órbita de $h$. E portanto $h$ é $k-\mathcal{R}_{\mathcal{X}^{-}}$ determinado.

Resta mostrar a existência de $\xi$.

Existe um homomorfismo natural entre os anéis $\mathcal{O}_{p} \rightarrow \mathcal{O}_{p+1}$. Se $I$ é um ideal em $\mathcal{O}_{p}$, denotaremos sua imagem em $\mathcal{O}_{p+1}$ por $I^{e}$ (ideal estendido, ver M.Atiyah [7]).

Consideremos

$$
A=\left\{\sum \frac{\partial H}{\partial y_{i}}(y, t) \cdot \xi_{t, i}(y) / \xi_{t} \in \operatorname{Der}(\log \mathcal{X}) \forall t\right\}
$$

Temos que $A$ é um ideal em $\mathcal{O}_{p+1}$.

Afirmamos que $\left(T \mathcal{R}_{\mathcal{X}} h\right)^{e} \subset A+\left(\mathcal{M}_{p}^{e}\right)^{k+1}$. De fato, se $\eta \cdot h \in T \mathcal{R}_{\mathcal{X}} h$ então

$$
\sum \frac{\partial H}{\partial y_{i}}(y, t) \cdot \eta_{i}(y)=\sum\left(\frac{\partial h}{\partial y_{i}}(y)+t \frac{\partial \hat{h}}{\partial y_{i}}(y)\right) \cdot \eta_{i}(y)=
$$




$$
=\sum \frac{\partial h}{\partial y_{i}}(y) \cdot \eta_{i}(y)+t \sum \frac{\partial \hat{h}}{\partial y_{i}}(y) \cdot \eta_{i}(y)
$$

Portanto $\left.\eta \cdot h=\sum \frac{\partial H}{\partial y_{i}}(y, t) \cdot \eta_{i}(y)-t \sum \frac{\partial \hat{h}}{\partial y_{i}}(y)\right) \cdot \eta_{i}(y)$ e a afirmação segue pois $\hat{h} \in \mathcal{M}_{p}^{k+1}$ e $\eta_{i} \in \mathcal{M}_{p}$.

Por hipótese $\mathcal{M}_{p}^{k+1} \subset \mathcal{M}_{p} T \mathcal{R}_{\mathcal{X}} h+\mathcal{M}_{p}^{k+2}$, então

$$
\begin{aligned}
\left(\mathcal{M}_{p}^{k+1}\right)^{e} & \subset \mathcal{M}_{p}^{e}\left(T \mathcal{R}_{\mathcal{X}} h\right)^{e}+\left(\mathcal{M}_{p}^{k+2}\right)^{e} \\
& \subset \mathcal{M}_{p}^{e}\left(A+\left(\mathcal{M}_{p}^{e}\right)^{k+1}\right)+\left(\mathcal{M}_{p}^{k+2}\right)^{e} \\
& \subset \mathcal{M}_{p+1} A+\mathcal{M}_{p+1}\left(\mathcal{M}_{p}^{e}\right)^{k+1}
\end{aligned}
$$

aplicando o Lema de Nakayama (ver M.Atiyah [7])

$$
\left(\mathcal{M}_{p}^{k+1}\right)^{e} \subset \mathcal{M}_{p+1} A \subset A
$$

Portanto $\hat{h} \in A$, ou seja, existe $\xi(y, t)=\xi_{t}(y), \quad \xi_{t} \in \operatorname{Der}(\log \mathcal{X})$, tal que $\sum \frac{\partial H}{\partial y_{i}}(y, t) \cdot \xi_{t, i}(y)=-\hat{h}(y)$.

Proposição 2.2.5 $S e \mathcal{M}_{p}^{k+1} \subset \mathcal{M}_{p} T \mathcal{R}_{\mathcal{X}} h+\mathcal{M}_{p}^{k+2}+h^{*} \mathcal{M}_{1} \cdot \mathcal{M}_{p}$ então $h$ é $k-\mathcal{K}_{\mathcal{X}}$-determinado.

Prova Como na Proposição acima, a prova segue análoga ao caso $\mathcal{K}$ (ver [33]).

Nosso objetivo a seguir é encontrar uma relação entre as $\mathcal{A}_{e}$-codimensões

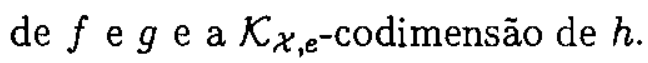

Teorema 2.2.6 Dados $\tilde{S}=\left\{z_{1}, \ldots, z_{r-1}\right\}$ e $S=\tilde{S} \cup\left\{z_{r}\right\}$ subconjuntos de $\mathbb{C}^{n}$, consideremos $f:\left(\mathbb{C}^{n}, \bar{S}\right) \rightarrow\left(\mathbb{C}^{n+1}, 0\right)$ um multigerme finitamente 
$\mathcal{A}$-determinado e $g:\left(\mathbb{C}^{n}, S\right) \rightarrow\left(\mathbb{C}^{n+1}, 0\right)$ satisfazendo $g^{(i)}=f^{(i)}$ para $i=$ $1, \ldots, r-1$ e $g^{(r)}$ é uma imersão. Se $h \in \mathcal{O}_{n+1}$ é uma equação reduzida que define a imagem de $g^{(r)}$ e $\mathcal{X}$ é a imagem de $f$, então a seguinte sequência é exata

$$
0 \rightarrow N \mathcal{K}_{\mathcal{X}, e} h \rightarrow N \mathcal{A}_{e} g \rightarrow N \mathcal{A}_{e} f \rightarrow 0
$$

A prova do Teorema 2.2.6 é baseada nos seguintes lemas.

Definição 2.2.7 Sejam $X$ e $Y$ variedades diferenciáveis e $f: X \rightarrow Y$ uma aplicação diferenciável $C^{\infty}$. Seja $\eta: Y \rightarrow T Y$ um campo vetorial em $Y$. Dizemos que $\eta$ admite um levantamento se existir $\xi: X \rightarrow T X$, campo vetorial em $X$, tal que $\omega f(\eta)=t f(\xi)$, em outras palavras, $\eta(f(x))=d f(\xi(x))$.

Lema 2.2.8 Seja $f:\left(\mathbb{C}^{n}, S\right) \rightarrow\left(\mathbb{C}^{n+1}, 0\right)$ um multigerme finitamente $\mathcal{A}$ determinado. Então o conjunto dos campos vetoriais que admitem levantamentos é igual a $\operatorname{Der}(\log (\mathcal{X}))$ onde $\mathcal{X}$ é a imagem de $f$.

Prova Consideremos $\eta \in \operatorname{Der}(\log \mathcal{X})$.

Como $f$ é finitamente determinado, $f$ é estável fora da origem; e as singularidades estáveis não isoladas são os cruzamentos normais. Logo podemos escolher um representante $f: U \subseteq\left(\mathbb{C}^{n}, S\right) \rightarrow\left(\mathbb{C}^{n+1}, 0\right)$ onde as únicas singularidades da imagem $f(U) \backslash\{0\}$ em $\mathbb{C}^{n+1}$ são cruzamentos normais.

Seja $\mathcal{X}_{0}$ o conjunto dos pontos onde $\mathcal{X}$ é $C^{\infty}, \mathcal{X}_{1}$ o conjunto dos pontos em $\mathcal{X}$ que são cruzamentos normais e $\mathcal{X}_{2}=\mathcal{X} \backslash\left(\mathcal{X}_{0} \cup \mathcal{X}_{1}\right)$.

Em $\mathbb{C}^{n} \backslash f^{-1}\left(\mathcal{X}_{2}\right)$ existe um único $\xi$ tal que $t f(\xi)=\omega f(\eta)$. Como a codimensão de $f^{-1}\left(\mathcal{X}_{2}\right)$ em $\mathbb{C}^{n}$ é maior ou igual a 2, segue pelo Teorema de Hartog que $\xi$ estende a um campo vetorial em $\mathbb{C}^{n}$. Como a equação $t f(\xi)=\omega f(\eta)$ vale em $\mathbb{C}^{n} \backslash f^{-1}\left(\mathcal{X}_{2}\right)$, uma vez que $f$ é estável, ela vale em $\mathbb{C}^{n}$. 
Reciprocamente, suponhamos que $\eta$ admita um levantamento. Seja $y \in$ $\mathcal{X}_{0}$. Então $f^{-1}(y)$ consiste de um único ponto $x$ e $\eta(y)=d f(\xi(x))$. Logo $\eta$ é tangente a $\mathcal{X}$ e portanto $\eta \in \operatorname{Der}(\log \mathcal{X})$.

Lema 2.2.9 Com as mesmas hipóteses do Teorema 2.2.6, a seguinte sequência é exata.

$$
0 \rightarrow \frac{\theta\left(g^{(r)}\right)}{t g^{(r)}(\theta(n))+\omega g^{(r)}(\operatorname{Der}(\log \mathcal{X}))} \rightarrow N \mathcal{A}_{e} g \rightarrow N \mathcal{A}_{e} f \rightarrow 0
$$

Prova Temos que

$$
N \mathcal{A}_{e} g=\frac{\theta(f) \oplus \theta\left(g^{(r)}\right)}{T \mathcal{A}_{e} g}
$$

Seja $A=T \mathcal{A}_{e} g \cap\left(0 \oplus \theta\left(g^{(r)}\right)\right)$. Segue do Lema 2.2.8 que

$$
\begin{gathered}
\frac{0 \oplus \theta\left(g^{(r)}\right)}{A} \cong \frac{0 \oplus \theta\left(g^{(r)}\right)}{0 \oplus\left(t g^{(r)}(\theta(n))+\omega g^{(r)}(\operatorname{Der}(\log \mathcal{X}))\right.} \cong \\
\frac{\theta\left(g^{(r)}\right)}{t g^{(r)}(\theta(n))+\omega g^{(r)}(\operatorname{Der}(\log \mathcal{X}))}
\end{gathered}
$$

A inclusão de $0 \oplus \theta\left(g^{(r)}\right)$ em $\theta(g)$ induz uma aplicação injetora

$$
\psi: \frac{0 \oplus \theta\left(g^{(r)}\right)}{A} \rightarrow N \mathcal{A}_{e} g
$$

A projeção de $\theta(g)=\theta(f) \oplus \theta\left(g^{(r)}\right)$ em $\theta(f)$ induz uma aplicação sobrejetora

$$
\phi: N \mathcal{A}_{e} g \rightarrow N \mathcal{A}_{e} f
$$

Resta mostrar que $\operatorname{Im} \psi=\operatorname{ker} \phi$.

Como $\phi \circ \psi=0$ temos $\operatorname{Im} \psi \subset \operatorname{ker} \phi$.

Reciprocamente, seja $(\omega, \nu) \in \theta(g)=\theta(f) \oplus \theta\left(g^{(r)}\right)$ e $[\omega, \nu]$ sua imagem no quociente $N \mathcal{A}_{e} g$. Suponhamos $\phi[\omega, \nu]=0$. Então $\omega \in T \mathcal{A}_{e} f$, ou seja, $\omega=t f(\xi)+\omega f(\eta)$ onde $\xi \in \theta(n)_{\tilde{s}}$ e $\eta \in \theta(n+1)$. Logo $(\omega, \nu)=$ 
$\left(t f(\xi)+\omega f(\eta), \omega g^{(r)}(\eta)\right)+\left(0, \nu-\omega g^{(r)}(\eta)\right)$ e $[\omega, \nu]=\left[\left(0, \nu-\omega g^{(r)}(\eta)\right)\right] \in \operatorname{Im} \psi$

Portanto $\operatorname{ker} \phi \subset \operatorname{Im} \psi$.

Lema 2.2.10 Com as mesmas hipóteses do Teorema 2.2.6,

$$
\frac{\theta\left(g^{(r)}\right)}{t g^{(r)}(\theta(n))+\omega g^{(r)}(\operatorname{Der}(\log \mathcal{X}))} \cong N \mathcal{K}_{\mathcal{X}, e} h
$$

Prova Sejam $x=\left(x_{1}, \ldots, x_{n}\right)$ e $y=\left(y_{1} \ldots, y_{n+1}\right)$ as coordenadas em $\mathbb{C}^{n}$ e $\mathbb{C}^{n+1}$ respectivamente. Como $g^{(r)}$ é uma imersão, após uma mudança de coordenadas podemos supor que $g^{(r)}(x)=(x, \lambda(x))$. Então $h(y)=y_{n+1}-$ $\lambda\left(y_{1}, \ldots, y_{n}\right)$.

Para facilitar a notaçāo, denotemos $T=t g^{(r)}(\theta(n))+\omega g^{(r)}(\operatorname{Der}(\log \mathcal{X}))$.

Seja $\omega \in \theta(h)$. Definimos $\phi: \theta(h) \rightarrow \theta\left(g^{(r)}\right)$ por $\phi(\omega)=\left(0, \ldots, 0, \omega\left(g^{(r)}\right)\right)$.

Temos que $\phi\left(T \mathcal{K}_{\mathcal{X}, e} h\right) \subset T$. De fato, se $\omega \in T \mathcal{K}_{\mathcal{X}, e} h$ então

$$
\omega=\sum_{i=1}^{n+1} \eta_{i} \frac{\partial h}{\partial y_{i}}+h \cdot \nu
$$

onde $\eta=\left(\eta_{1}, \ldots, \eta_{n+1}\right) \in \operatorname{Der}(\log \mathcal{X})$ e $\nu \in \theta(h)$. Consideremos $\xi=$ $\left(\xi_{1}, \ldots, \xi_{n}\right) \in \theta(n)$ dado por $\xi_{i}(x)=-\eta_{i}(x, \lambda(x))$ para $i=1, \ldots, n$. Logo $\phi(\omega)=t g^{(r)}(\xi)+\omega(\eta) \in T$. Portanto temos uma aplicação bem definida

$$
\phi: N \mathcal{K}_{\mathcal{X}, e} h \rightarrow \frac{\theta\left(g^{(r)}\right)}{T}
$$

Se $\omega \in \theta(h)$ denotemos por $[\omega]$ sua imagem em $N \mathcal{K}_{\mathcal{X}, e} h$. Suponhamos $\phi[\omega]=0$. Logo existem $\hat{\xi}=\left(\hat{\xi}_{1}, \ldots, \hat{\xi}_{n}\right) \in \theta(n)$ e $\hat{\eta}=\left(\hat{\eta}_{1}, \ldots, \hat{\eta}_{n+1}\right) \in$ $\operatorname{Der}(\log \mathcal{X}))$ tais que

$$
\left(0, \ldots, 0, \omega\left(g^{(r)}\right)\right)=t g^{(r)}(\hat{\xi})+\omega g^{(r)}(\hat{\eta})
$$

ou seja,

$$
\left(0, \ldots, 0, \omega\left(g^{(r)}\right)\right)=\left(\hat{\xi}_{1}, \ldots, \hat{\xi}_{n}, \sum_{i=1}^{n} \hat{\xi}_{i} \frac{\partial \lambda}{\partial x_{i}}\right)+\hat{\eta}\left(g^{(r)}\right)
$$


portanto

$$
\omega\left(g^{(r)}\right)=\hat{\eta}_{n+1}\left(g^{(r)}\right)-\sum_{i=1}^{n} \hat{\eta}_{i}\left(g^{(r)}\right) \frac{\partial \lambda}{\partial x_{i}}
$$

Segue que $\hat{\nu}=\omega-\eta_{n+1}+\sum \eta_{i} \frac{\partial \lambda}{\partial y_{i}}$ é uma equação que define a imagem de $g^{(r)}$. Assim $\hat{\nu} \in h^{*} \mathcal{M}_{1}$ e $\omega \in T \mathcal{K}_{\mathcal{X}, e} h$. Portanto $\phi$ é injetora

Temos que $\phi$ é sobrejetora pois dado $\zeta=\left(\zeta_{1}, \ldots, \zeta_{n+1}\right) \in \theta\left(g^{(r)}\right)$, seja $\rho(y)=\zeta_{n+1}\left(y_{1}, \ldots, y_{n}\right)-\sum_{i=1}^{n} \frac{\partial \lambda}{\partial y_{i}} \zeta_{i}\left(y_{1}, \ldots, y_{n}\right) \in \theta(h)$. Então

$$
\left(0, \ldots, 0, \rho\left(g^{(r)}\right)\right)=\left(\zeta_{1}, \ldots, \zeta_{n+1}\right)-\left(\zeta_{1}, \ldots, \zeta_{n}, \sum_{i=1}^{n} \frac{\partial \lambda}{\partial x_{i}} \zeta_{i}\right)
$$

e portanto $\phi[\rho]=[\zeta]$.

Corolário 2.2.11 Com as mesmas hipóteses do Teorema 2.2.6, se f é estável então $N \mathcal{K}_{\mathcal{X}, e} h$ é isomorfo a $N \mathcal{A}_{e} g$.

Observaçāo 2.2.12 Teorema 2.2.6 não é verdadeiro se $g^{(r)}$ não é imersão. Por exemplo seja $f(x, y)=(x, x, y)$ e $g^{(2)}(x, y)=\left(x, y^{2}, x y\right)$. Então se $g$ é um bigerme cujos ramos são $f$ e $g^{(2)}, g$ tem $\mathcal{A}_{e}$-codimensāo 1 e $h(X, Y, Z)=$ $Z^{2}-X^{2} Y$ tem $\mathcal{K}_{\mathcal{X}, e^{-c o d i m e n s a ̃ o ~ i n f i n i t a . ~}}$

Para calcular a $\mathcal{A}$-codimensão temos o seguinte resultado devido a Leslie Wilson [18].

Proposição 2.2.13 Seja $S=\left\{z_{1}, \ldots, z_{r}\right\}$ e $f:\left(\mathbb{C}^{n}, S\right) \rightarrow\left(\mathbb{C}^{p}, 0\right)$ um multigerme $\mathcal{A}$-finito. Se $f$ é estável a $\mathcal{A}_{e}$-codimensão é zero. Se a $\mathcal{A}_{e}$-codimensão é estritamente maior que zero então temos a seguinte relação:

$$
\mathcal{A}_{e}-\text { codimensão }=\mathcal{A}-\text { codimensão }+r(p-n)-p
$$




\subsection{Classificação de bigermes}

No que segue denotaremos por $(x, y)$ e $(X, Y, Z)$ as coordenadas em $\mathbb{C}^{2}$ e $\mathbb{C}^{3}$ respectivamente.

O seguinte resultado foi obtido por David Mond [26].

Proposição 2.3.1 (i) Seja $f$ um bigerme $(|S|=2)$ de $\mathbb{C}^{2}$ em $\mathbb{C}^{3}$, onde cada ramo é uma imersão. Então $f$ é $\mathcal{A}$-equivalente a

$$
\left\{\begin{aligned}
(x, y) & \mapsto(x, y, 0) \\
(x, y) & \mapsto(x, y, \varphi(x, y))
\end{aligned}\right.
$$

onde $\varphi$ é chamada função de contato.

(ii) Dois bigermes da forma (i) são $\mathcal{A}$-equivalentes se, e somente se, as funções $\varphi$ correspondentes são $\mathcal{K}$-equivalentes.

Corolário 2.3.2 Um bigerme da forma dada pela Proposição 2.3.1(i) é simples se, e somente se, a função de contato $\varphi$ correspondente é simples, ou seja $\varphi$ é uma das seguintes (ver [4]):

$$
\begin{aligned}
& A_{k}: \quad \varphi(x, y)=x^{2}+y^{k+1} \quad k \geq 1 \\
& D_{k}: \quad \varphi(x, y)=x^{2} y+y^{k-1} \quad k \geq 4 \\
& E_{6}: \quad \varphi(x, y)=x^{3}+y^{4} \\
& E_{7}: \quad \varphi(x, y)=x^{3}+x y^{3} \\
& E_{8}: \quad \varphi(x, y)=x^{3}+y^{5}
\end{aligned}
$$


Consideraremos agora os bigermes onde um dos ramos é um cross-cap.

Proposiçāo 2.3.3 Seja $f:\left(\mathbb{C}^{2}, S\right) \rightarrow\left(\mathbb{C}^{3}, 0\right)$ um bigerme onde um dos seus ramos é um cross-cap. Então $f$ é $\mathcal{A}$-equivalente a um dos seguintes $(k \geq 1)$ :

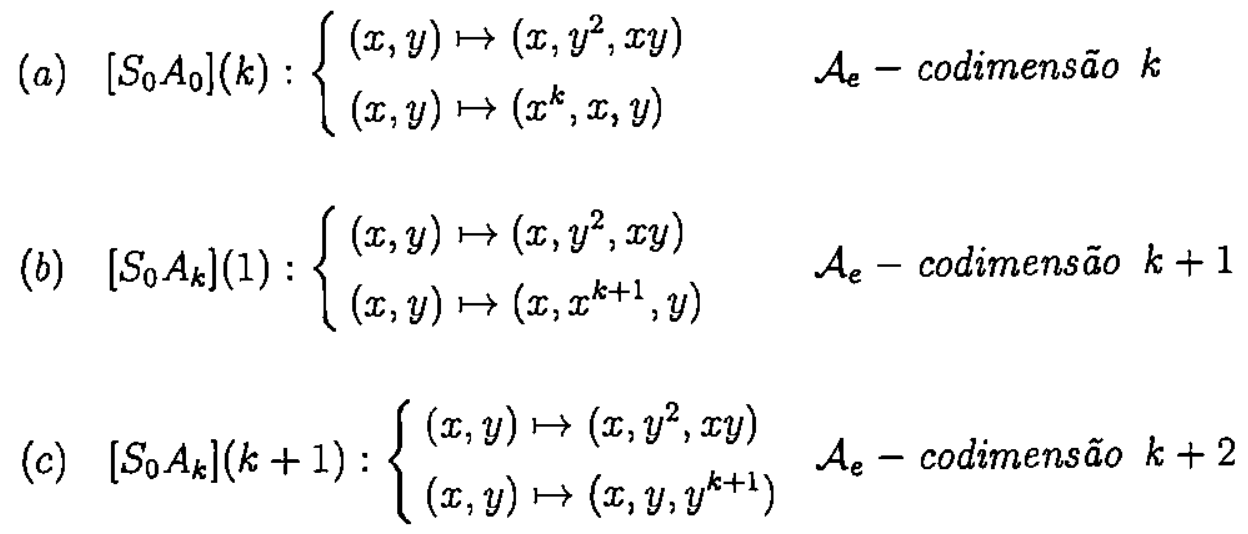

Prova I. Classificação.

Seja $\mathcal{X}$ a imagem de um cross cap. Suponhamos $\mathcal{X}=\left\{(X, Y, Z) / Z^{2}-\right.$ $\left.X^{2} Y=0\right\}$. Então $\operatorname{Der}(\log \mathcal{X})=<(X,-2 Y, 0),\left(0,2 Z, X^{2}\right),(X, 0, Z),(Z, 0$, $X Y)>\theta(3)$.

Segue do Teorema 2.2.1 que as $\mathcal{A}$-classes de tais bigermes são dadas pelas $\mathcal{K}_{\mathcal{X}}$-classes em $\mathcal{O}_{3}$.

Consideremos primeiramente a ação de $G=J^{1} \mathcal{K}_{\mathcal{X}}$ em $M=J^{1}(3,1)$. Seja $\sigma=a X+b Y+c Z$ um elemento em $M$. Segue do Lema 2.2.3 que

$$
T_{\sigma} J^{1} \mathcal{K}_{\mathcal{X}} \cdot \sigma=<a X, a Z, b Y, b Z, c Z>\mathcal{O}_{3}\left(\bmod \mathcal{M}_{3}^{2}\right)
$$

Seja $S=\{\sigma \in M / a \neq 0, b \neq 0\}$. Segue do Lema de Mather que $S$ está contida numa única $G$-órbita.

Prosseguindo desta maneira obtemos 5 órbitas em $J^{1}(3,1)$ : aquelas de

$$
X-Y, \quad X, \quad Y, \quad Z, \quad 0
$$


Se $h(X, Y, Z)=X-Y$, segue de 2.2 .5 que $h$ é $1-\mathcal{K}_{\mathcal{X}}$-determinado e tem $\mathcal{K}_{\mathcal{X}, e^{- \text {codimensão }} 1 .}$

Consideremos agora germes cujo 1-jato é $X, Y$ ou $Z$.

Sejam

$$
\begin{aligned}
& \sigma_{1}(X, Y, Z)=X+\sum_{i+j+l=k} \alpha_{i, j, l} X^{i} Y^{j} Z^{l} \\
& \sigma_{2}(X, Y, Z)=Y+\sum_{i+j+l=k} \beta_{i, j, l} X^{i} Y^{j} Z^{l} \\
& \sigma_{3}(X, Y, Z)=Z+\sum_{i+j+l=k} \gamma_{i, j, l} X^{i} Y^{j} Z^{l}
\end{aligned}
$$

elementos em $J^{k}(3,1)$.

Então

$$
\begin{aligned}
& T_{\sigma_{1}} J^{k} \mathcal{K}_{\mathcal{X}} \cdot \sigma_{1}=<X, \alpha_{0, k, 0} Y^{k}, Z>\mathcal{O}_{3}\left(\bmod \mathcal{M}_{3}^{k+1}\right) \\
& T_{\sigma_{2}} J^{k} \mathcal{K}_{\mathcal{X}} \cdot \sigma_{2}=<\beta_{k, 0,0} X^{k}, Y, Z>\mathcal{O}_{3}\left(\bmod \mathcal{M}_{3}^{k+1}\right) \\
& T_{\sigma_{3}} J^{k} \mathcal{K}_{\mathcal{X}} \cdot \sigma_{3}=<X^{2}, X Y, \gamma_{0, k, 0} Y^{k}, Z>\mathcal{O}_{3}\left(\bmod \mathcal{M}_{3}^{k+1}\right)
\end{aligned}
$$

Analogamente temos as seguintes $J^{k} \mathcal{K}_{\mathcal{X}}$-órbitas em $J^{k}(3,1)$ : aquelas de $X-Y^{k}, Y-X^{k}, Z-Y^{k}(k \geq 2)$.

Sejam

$$
\begin{aligned}
& h_{1}(X, Y, Z)=X-Y^{k} \\
& h_{2}(X, Y, Z)=Y-X^{k} \\
& h_{3}(X, Y, Z)=Z-Y^{k}
\end{aligned}
$$

segue de 2.2.5 que $h_{i}$ é $k-\mathcal{K}_{\mathcal{X}}$-determinado para $i=1,2,3$. Também $h_{1} \mathrm{e}$

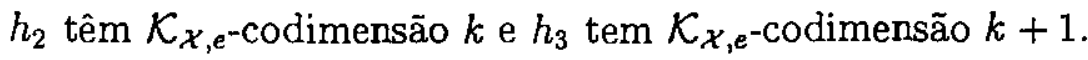

Portanto as $\mathcal{A}$-classes de tais bigermes e suas codimensões seguem dos Teoremas 2.2.1 e 2.2.6. Resta mostrar que se $h$ tem 1-jato igual a $0, h$ não pode ser simples. De fato, seja $M$ o espaço das formas quadráticas em $\mathbb{C}^{3}$. A dimensão de $M$ é 6. Se $\sigma \in M$ então

$$
\operatorname{dim} T_{\sigma} J^{2} \mathcal{K}_{\mathcal{X}} \sigma \leq 5
$$


Portanto a dimensão das órbitas de $J^{2} \mathcal{K}_{\mathcal{X}}$ em $M$ é menor que a dimensão de $M$. Logo as órbitas formam uma família continua em $M$. Consequentemente, $h$ nāo é $\mathcal{K}_{\mathcal{X}}$-simples.

Este argumento de contar dimensões foi usado por V.I.Arnold [4][Lema 4.2] para mostrar que o coposto de um germe de função simples não excede 2.

II. Os bigermes classificados em I são simples:

(a) O espaço tangente a $\left[S_{0} A_{0}\right](k)$ segundo o grupo $\mathcal{A}$ é dado por

$$
\begin{gathered}
\left(\begin{array}{cc}
\mathcal{M}_{2}-\left\{y^{2}, \ldots, y^{2 k-2}\right\} & \mathcal{M}_{2}-\left\{x, \ldots, x^{k-1}\right\} \\
\mathcal{M}_{2}-\{y\} & \mathcal{M}_{2} \\
\mathcal{M}_{2}-\left\{y, \ldots, y^{2 k-1}\right\} & \mathcal{M}_{2}
\end{array}\right) \\
+\mathbb{C}\left\{\left(\begin{array}{cc}
y^{2 i} & 0 \\
0 & 0 \\
y^{2 i+1} & 0
\end{array}\right),\left(\begin{array}{cc}
y^{2 i} & x^{i} \\
0 & 0 \\
0 & 0
\end{array}\right)\right\}_{i=1, \ldots, k-1}
\end{gathered}
$$

Usando o Teorema da Versalidade [35, p.499] temos que um desdobramento $\mathcal{A}$-versal de $\left[S_{0} A_{0}\right](k)$ é dado por

$$
f_{u}:\left\{\begin{array}{l}
(x, y) \mapsto\left(x, y^{2}+u_{k} y, x y+u_{k+1} y\right) \\
(x, y) \mapsto\left(x^{k}+u_{k-1} x^{k-1}+\cdots+u_{1} x, x, y\right)
\end{array}\right.
$$

Se $u_{k} \neq 0$ ou $u_{k+1} \neq 0$ então $f_{u}$ consiste de duas imersões que são transversais. Portanto $f_{u}$ é $\mathcal{A}$-equivalente ao ponto duplo ordinário:

$$
f:\left\{\begin{aligned}
(x, y) & \mapsto(x, y, 0) \\
(x, y) & \mapsto(x, 0, y)
\end{aligned}\right.
$$

Suponhamos $u_{k}=u_{k+1}=0$. Então $h(X, Y, Z)=X-u_{1} Y-\cdots-u_{k-1} Y^{k-1}-$ $Y^{k}$ é uma equação que define a imagem do segundo ramo de $f_{u}$. 
Se $u_{1}=\cdots=u_{i-1}=0$ e $u_{i} \neq 0$ então $j^{i} h(0)=X-u_{i} Y^{i}$. Segue da classificação que $h$ é $\mathcal{K}_{\mathcal{X}}$-equivalente a $X-Y^{i}$.

Portanto $f_{u}$ é $\mathcal{A}$-equivalente a $\left[S_{0} A_{0}\right](i)$, para $i<k$.

(b) O espaço tangente a $\left[S_{0} A_{k}\right](1)$ segundo o grupo $\mathcal{A}$ é dado por

$$
\left(\begin{array}{cc}
\mathcal{M}_{2} & \mathcal{M}_{2} \\
\mathcal{M}_{2}-\left\{y, x, x^{2}, \ldots, x^{k}\right\} & \mathcal{M}_{2}-\left\{x, \ldots, x^{k}\right\} \\
\mathcal{M}_{2}-\{y\} & \mathcal{M}_{2}
\end{array}\right)+\mathbb{C}\left\{\left(\begin{array}{cc}
0 & 0 \\
x^{i} & x^{i} \\
0 & 0
\end{array}\right)\right\}_{i=1, \ldots, k}
$$

Logo, um desdobramento $\mathcal{A}$-versal de $\left[S_{0} A_{k}\right](1)$ é dado por

$$
g_{u}:\left\{\begin{array}{l}
(x, y) \mapsto\left(x, y^{2}+u_{k+1} y, x y+u_{k+2} y\right) \\
(x, y) \mapsto\left(x, x^{k+1}+u_{k} x^{k}+\cdots+u_{1} x, y\right)
\end{array}\right.
$$

Se $u_{k+1} \neq 0$ ou $\left(u_{k+2} \neq 0\right.$ e $\left.u_{1} \neq 0\right)$ então $g_{u}$ consiste de duas imersões transversais.

Suponhamos $u_{k+1}=u_{1}=0$ e $u_{k+2} \neq 0$. Então $g_{u}$ consiste de duas imersões que são tangentes na origem. Portanto $g_{u}$ é $\mathcal{A}$-equivalente.a

$$
\left\{\begin{aligned}
(x, y) & \mapsto(x, y, 0) \\
(x, y) & \mapsto(x, y, \varphi(x, y))
\end{aligned}\right.
$$

onde

$$
\varphi(x, y)=\frac{y^{2}}{\left(x+u_{k+2}\right)^{2}}-x^{k+1}-u_{k} x^{k}-\cdots-u_{2} x^{2}
$$

Segundo C.Gibson [14, p.129, Teorema 4.6], $\varphi$ é $\mathcal{R}$-equivalente a $x^{\ell}+y^{2}$ onde $\ell \leq k+1$. Portanto $g_{u}$ é $\mathcal{A}$-equivalente a

$$
\left[A_{\ell-1}\right]:\left\{\begin{aligned}
(x, y) & \mapsto(x, y, 0) \\
(x, y) & \mapsto\left(x, y, x^{2}+y^{\ell}\right)
\end{aligned}\right.
$$

Também, análogo a (a), temos que $g_{u}$ é $\mathcal{A}$-equivalente a $\left[S_{0} A_{i}\right](1)$, para $\operatorname{algum} i<k$, ou a $\left[S_{0} A_{0}\right](1)$. 
(c) O espaço tangente a $\left[S_{0} A_{k}\right](k+1)$ segundo o grupo $\mathcal{A}$ é dado por

$$
\begin{gathered}
\left(\begin{array}{cc}
\mathcal{M}_{2}-\left\{y, y^{3}, \ldots, y^{2 k-1}\right\} & \mathcal{M}_{2} \\
\mathcal{M}_{2}-\{y\} & \mathcal{M}_{2} \\
\mathcal{M}_{2}-\left\{x, y, y^{2}, \ldots, y^{2 k}\right\} & \mathcal{M}_{2}-\left\{y, y^{2}, \ldots, y^{k}\right\}
\end{array}\right)+ \\
+\mathbb{C}\left\{\left(\begin{array}{cc}
y^{2 i-1} & 0 \\
0 & 0 \\
y^{2 i} & 0
\end{array}\right),\left(\begin{array}{cc}
0 & 0 \\
0 & 0 \\
y^{2 i} & y^{i}
\end{array}\right)\right\}_{i=1, \ldots, k}
\end{gathered}
$$

Logo, um desdobramento $\mathcal{A}$-versal de $\left[S_{0} A_{k}\right](k+1)$ é dado por

$$
\tilde{f}_{u}:\left\{\begin{array}{l}
(x, y) \mapsto\left(x, y^{2}+u_{k+1} y, x y+u_{k+2} x+u_{k+3} y\right) \\
(x, y) \mapsto\left(x, y, y^{k+1}+u_{k} y^{k}+\cdots+u_{1} y\right)
\end{array}\right.
$$

Se $u_{k+2} \neq 0$ ou $u_{k+3} \neq 0$ ou $\left(u_{k+1} \neq 0\right.$ e $\left.u_{1} \neq 0\right)$ então $\tilde{f}_{u}$ consiste de duas imersões transversais.

Suponhamos $u_{k+2}=u_{k+3}=u_{1}=0$ e $u_{k+1} \neq 0$. Então $\tilde{f}_{u}$ é $\mathcal{A}$-equivalente a

$$
\left\{\begin{array}{l}
(x, y) \mapsto(x, y, 0) \\
(x, y) \mapsto(x, y, \varphi(x, y))
\end{array}\right.
$$

onde $\varphi(x, y)=x \phi(y)-y^{k+1}-u_{k} y^{k}-\cdots-u_{2} y^{2}$. Como $j^{2} \varphi(0)=\frac{x y}{u_{k+1}}-u_{2} y^{2}$, $\varphi$ é $\mathcal{R}$-equivalente a $x^{2}-y^{2}$. Portanto $\tilde{f}_{u}$ é $\mathcal{A}$-equivalente a $\left[A_{1}\right]$.

Analogamente, $\tilde{f}_{u}$ é $\mathcal{A}$-equivalente a $\left[S_{0} A_{i}\right](i+1), i<k$, ou $\tilde{f}_{u}$ é $\mathcal{A}$ equivalente a $\left[S_{0} A_{1}\right](1)$.

Observação 2.3.4 O bigerme $\left[S_{0} A_{0}\right](k)$ é $\mathcal{A}$-equivalente ao bigerme classificado por D.Mond [26]:

$$
\left\{\begin{array}{l}
(x, y) \mapsto(x, y, 0) \\
(x, y) \mapsto\left(y^{2}, x y+y^{2 k+1}, x\right)
\end{array}\right.
$$

É o único bigerme em 2.3.3 cujos ramos são transversais. 
Definição 2.3.5 Dizemos que a $\mathcal{A}$-classe $L$ de singularidades é adjacente $\grave{a} \mathcal{A}$-classe $K$, e denotamos por $L \rightarrow K$, se todo multigerme $f \in L$ pode ser deformado em um multigerme em $K$ por uma pertubação arbitrariamente pequena. Isto é equivalente a dizer que $K$ especializa $L$. Assim, as $\mathcal{A}$-classes às quais $L$ é adjacente são aquelas que aparecem num desdobramento $\mathcal{A}$-versal de qualquer multigerme em $L$.

Proposição 2.3.6 Seja $f:\left(\mathbb{C}^{2}, S\right) \rightarrow\left(\mathbb{C}^{3}, 0\right)$ um bigerme simples onde um dos seus ramos é $S_{k}(k \geq 0)$, então $f$ é $\mathcal{A}$-equivalente a

$$
\left[S_{k} A_{0}\right]:\left\{\begin{array}{l}
(x, y) \mapsto\left(x, y^{2}, y^{3}+x^{k+1} y\right) \\
(x, y) \mapsto(0, x, y)
\end{array}\right.
$$

Prova Seguiremos a prova de 2.3.3.

Seja $\mathcal{X}$ a imagem de $S_{k}$. Então $\operatorname{Der}(\log \mathcal{X})=<(2 X, 2(k+1) Y, 3(k+$ 1) $Z),\left(0,2 Z, X^{2 k+2}+4 X^{k+1} Y+3 Y^{2}\right),\left(X^{k+1}+3 Y,-2(k+1) X^{k} Y, 0\right),(Z, 0,(k+$ 1) $\left.\left(X^{2 k+1} Y+X^{k} Y^{2}\right)\right)>\theta(3)$.

Calculando o espaço tangente e aplicando o Lema de Mather temos que as $J^{1} \mathcal{K}_{\mathcal{X}}$-órbitas em $J^{1}(3,1)$ são aquelas de:

$$
X, Y, Z, 0
$$

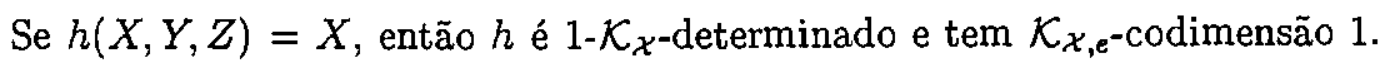
Suponhamos $k=1$.

Em $J^{2}(3,1)$ as $J^{2} \mathcal{K}_{\mathcal{X}}$-órbitas de elementos cujo 1-jato é $Y$ são aquelas de

$$
h_{\alpha}=Y+\alpha X^{2}
$$

Como $X^{2} \notin T_{h_{\alpha}} J^{2} \mathcal{K}_{\chi} h_{\alpha}$, segue que $h_{\alpha}$ é modal.

Em $J^{2}(3,1)$ as $J^{2} \mathcal{K}_{\mathcal{X}}$-órbitas de elementos cujo 1-jato é $Z$ são aquelas de

$$
Z-X^{2}, \quad Z-\beta X Y
$$


aqui novamente temos modalidade: afirmamos que

$$
g:\left\{\begin{array}{l}
(x, y) \mapsto\left(x, y^{2}, y^{3}+x^{2} y\right) \\
(x, y) \mapsto\left(x, y, x^{2}\right)
\end{array}\right.
$$

não é $\mathcal{A}$-simples.

De fato, seja

$$
g_{u}:\left\{\begin{array}{l}
(x, y) \mapsto\left(x, y^{2}, y^{3}+x^{2} y\right) \\
(x, y) \mapsto\left(x, y, x^{2}+u y\right)
\end{array}\right.
$$

uma perturbação de $g$. Seja $h(X, Y, Z)=Z-u Y-X^{2}$ uma equação que define o segundo ramo de $g_{u}$. Segue da classificação acima que $h$ não é $\mathcal{K}_{\mathcal{X}^{-}}$ simples. Portanto $g_{u}$ não é $\mathcal{A}$-simples. Logo $g$ não é simples pois é adjacente a um bigerme não simples.

Se $k>1$, como $S_{k}$ é adjacente a $S_{1}$, o único bigerme simples é $\left[S_{k} A_{0}\right]$.

Com o resultado seguinte completamos a classificação de bigermes simples de $\mathbb{C}^{2}$ em $\mathbb{C}^{3}$.

Proposição 2.3.7 Um bigerme de $\mathbb{C}^{2}$ em $\mathbb{C}^{3}$ onde um de seus ramos é $B_{k}$ ou $H_{k}, k \geq 2$, não é simples.

Prova (a) Seja $\mathcal{X}$ a imagem de $f_{1}(x, y)=\left(x, y^{2}, x^{2} y+y^{5}\right)$. Então $\operatorname{Der}(\log \mathcal{X})=<(2 X, 2 Y, 5 Z),\left(X^{2}+5 Y^{2},-4 X Y, 0\right),\left(0,2 Z, X^{4}+6 X^{2} Y^{2}+\right.$ $\left.5 Y^{4}\right),\left(Z, 0,2 X^{3} Y+2 X Y^{3}\right)>\theta(3)$.

Se $\sigma=a X+b Y+c Z \in J^{1}(3,1)$ então

$$
T_{\sigma} J^{1} \mathcal{K}_{\mathcal{X}} \cdot \sigma=<a X+b Y, a Z, b Z, c Z>\mathcal{O}_{3}\left(\bmod \mathcal{M}_{3}^{2}\right)
$$

Portanto a dimensão de qualquer órbita de $J^{1} \mathcal{K}_{\mathcal{X}}$ em $J^{1}(3,1)$ é menor que a dimensão de $J^{1}(3,1)$. Consequentemente um germe em $\mathcal{O}_{3}$ não é $\mathcal{K}_{\mathcal{X}}$-simples. 
Existe também um argumento geométrico. Consideremos o bigerme

$$
f:\left\{\begin{aligned}
(x, y) & \mapsto\left(x, y^{2}, x^{2} y-y^{5}\right) \\
(x, y) & \mapsto(x, y, a x+b y)
\end{aligned}\right.
$$

Consistindo do germe de uma imersão e do germe do $B_{2}$ se interceptando transversalmente. Afirmamos que $a$ e $b$ são parâmetros modais. Se fato, seja $\varphi \in \mathcal{R}_{\mathcal{X}}$ tal que $\varphi(\{Z-a X-b Y=0\})=\{Z-\tilde{a} X-\tilde{b} Y=0\}$.

Os planos $X=0$ e $Z=0$ sāo planos de simetria de $\mathcal{X}$. Como $d \varphi(0)$ leva plano de simetria em plano de simetria, $d \varphi(0)$ fixa o plano $Z=0$, pois não podemos ter $d \varphi(0)(\{Z=0\})=\{X=0\}$.

No plano $Z=0$ temos três subespaços lineares que são preservados por $d \varphi(0)$ : o cone tangente a $\mathcal{X}_{\text {sing }}$, ou seja, as retas $X \pm Y=0$, e $d \varphi(0)(\mathbb{C})$.

Logo $\{Z-a X-b Y=0\} \cap\{Z=0\}$ é a quarta reta e seu cross-ratio com as outras três é um invariante de $d \varphi(0)$. Portanto $f$ não é simples.

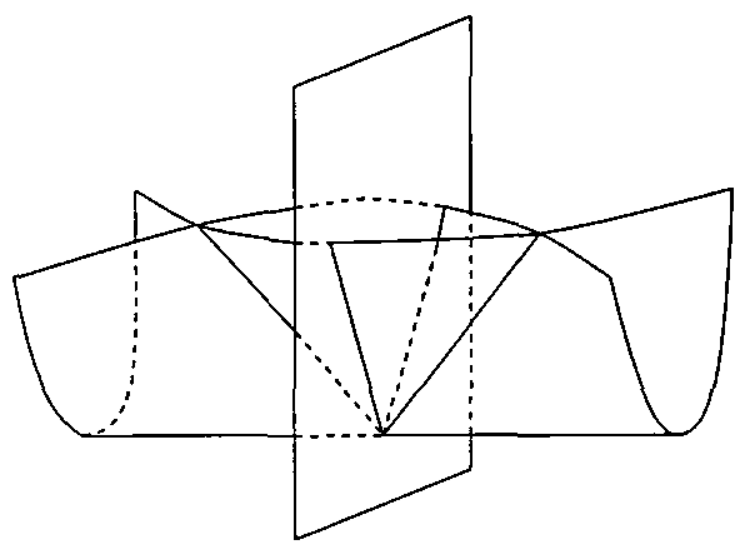

Figura 2.1: Intersecçāo do $B_{2}$ com um plano 
Mais geralmente, não existe bigerme simples onde um dos ramos é $B_{k}$, $C_{k}$ ou $F_{4}$ pois estes últimos são adjacentes a $f_{1}\left(B_{2}\right)$.

(b) Seja $\mathcal{X}$ a imagem de $g_{1}(x, y)=\left(x, y^{3}, x y+y^{5}\right)$. Então $\operatorname{Der}(\log \mathcal{X})=<$ $(4 X, 3 Y, 5 Z),\left(X^{2}+5 Y Z,-3 X Y, 5 Y^{3}\right),\left(5 Y^{3}+X Z,-3 Y Z,-5 X Y^{2}\right),\left(9 X Y^{2}-\right.$ $\left.5 Z^{2}, 3 Y^{3},-5 X^{2} Y\right),\left(29 X^{2} Y+25 Y^{2} Z, 18 X Y^{2}-15 Z^{2},-5 X^{3}\right)>\theta(3)$.

Se $\sigma=a X+b Y+c Z \in J^{1}(3,1)$ então

$$
T_{\sigma} J^{1} \mathcal{K}_{\mathcal{X}} \cdot \sigma=<a X+b Y+c Z, 4 a X+3 b Y+5 c Z>\mathcal{O}_{3}\left(\bmod \mathcal{M}_{3}^{2}\right)
$$

Como em (a), não existe germe em $\mathcal{O}_{3}$ que é $\mathcal{K}_{\mathcal{X}}$-simples.

\subsection{Classificação de trigermes}

Lema 2.4.1 Um trigerme $(|S|=3)$ de $\mathbb{C}^{2}$ em $\mathbb{C}^{3}$ cujos ramos são imers̃oes não duas a duas tangentes, é $\mathcal{A}$-equivalente a

$$
f:\left\{\begin{aligned}
(x, y) & \mapsto(x, y, 0) \\
(x, y) & \mapsto(x, 0, y) \\
(x, y) & \mapsto\left(x, y, f_{13}(x, y)\right)
\end{aligned}\right.
$$

Prova Segue do fato que podemos escolher coordenadas na meta tais que os planos tangentes às imersões são $Y=0, Z=0$ e um outro plano que se projeta bijetivamente sobre $Z=0$.

Seja $\mathcal{R}^{\delta}$ um subgrupo de $\mathcal{R}$ consistindo de germes de difeomorfismos $\varphi$ : $\left(\mathbb{C}^{2}, 0\right) \mapsto\left(\mathbb{C}^{2}, 0\right), \varphi=\left(\varphi_{1}, \varphi_{2}\right)$, que fixa a reta $y=0$, ou seja, $\varphi_{2}(x, 0)=0$. Consideremos $\mathcal{K}^{\delta}=\mathcal{R}^{\delta} \cdot \mathcal{C}$ (produto semi-direto). Então

$$
T \mathcal{K}_{e}^{\delta} f_{13}=<\frac{\partial f_{13}}{\partial x}, y \frac{\partial f_{13}}{\partial y}, f_{13}>\mathcal{O}_{2}
$$


O resultado seguinte mostra que a $\mathcal{A}$-classificação de tais trigermes é equivalente à classificação de germes de funções numa variedade com bordo com respeito a $\mathcal{K}^{\delta}(\operatorname{ver}[3],[26])$.

Proposição 2.4.2 Sejam

$$
f:\left\{\begin{array}{l}
(x, y) \stackrel{f_{3}}{\rightarrow}(x, y, 0) \\
(x, y) \stackrel{f_{2}}{\rightarrow}(x, 0, y) \\
(x, y) \stackrel{f_{3}}{\rightarrow}\left(x, y, f_{13}(x, y)\right)
\end{array} \quad e \quad \bar{f}:\left\{\begin{array}{l}
(x, y) \stackrel{f_{3}}{\rightarrow}(x, y, 0) \\
(x, y) \stackrel{f_{2}}{\rightarrow}(x, 0, y) \\
(x, y) \stackrel{f_{3}}{\rightarrow}\left(x, y, \bar{f}_{13}(x, y)\right)
\end{array}\right.\right.
$$

então

(i) f é $\mathcal{A}$-equivalente a $\bar{f}$ se, e somente se, $f_{13}$ é $\mathcal{K}^{\delta}$-equivalente a $\bar{f}_{13}$.

(ii) Se f é finitamente $\mathcal{A}$-determinado

$$
\begin{aligned}
& T \mathcal{A}_{e} f=\left(\begin{array}{ccc}
\mathcal{O}_{2} & \mathcal{O}_{2} & \mathcal{O}_{2} \\
\mathcal{O}_{2} & \mathcal{O}_{2}-\left\{1, x, \ldots, x^{N}\right\} & \mathcal{O}_{2}-\left\{1, x, \ldots, x^{N}\right\} \\
T \mathcal{K}_{e}^{\delta} f_{13} & \mathcal{O}_{2} & T \mathcal{K}_{e}^{\delta} f_{13}
\end{array}\right)+
\end{aligned}
$$

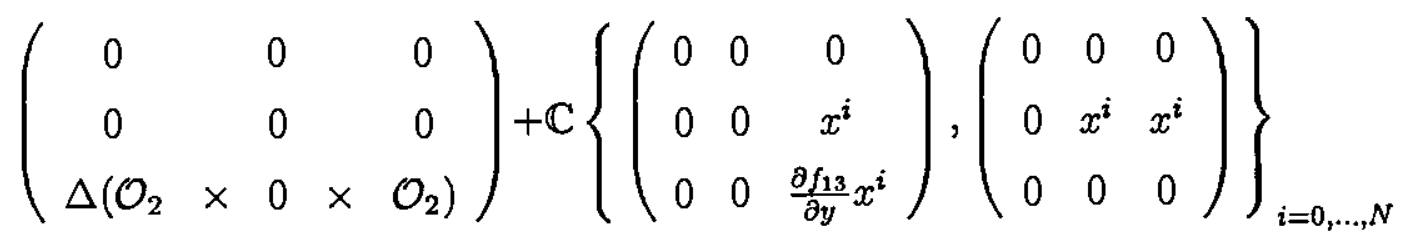

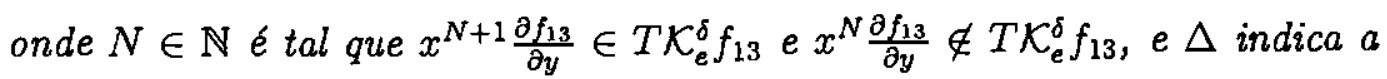
diagonal de $\mathrm{O}_{2} \times\{0\} \times \mathcal{O}_{2}$.

Prova (i) Suponhamos $f \mathcal{A}$-equivalente a $\bar{f}$.

Então existem germes de difeomorfismos $\varphi_{i}:\left(\mathbb{C}^{2}, 0\right) \rightarrow\left(\mathbb{C}^{2}, 0\right), \varphi_{i}=$ $\left(\varphi_{i 1}, \varphi_{i 2}\right), i=1,2,3$, e $\psi:\left(\mathbb{C}^{2}, 0\right) \rightarrow\left(\mathbb{C}^{3}, 0\right), \psi=\left(\psi_{1}, \psi_{2}, \psi_{3}\right)$, tais que

(1) $\psi \circ f_{1} \circ \varphi_{1}^{-1}=\bar{f}_{1}$.

(2) $\psi \circ f_{2} \circ \varphi_{2}^{-1}=\bar{f}_{2}$. 
(3) $\psi \circ f_{3} \circ \varphi_{3}^{-1}=\bar{f}_{3}$.

Seja $K:\left(\mathbb{C}^{2} \times \mathbb{C},(0,0)\right) \rightarrow\left(\mathbb{C}^{2} \times \mathbb{C},(0,0)\right)$ dado por $K(x, y, t)=\left(\varphi_{3}(x, y)\right.$, $\left.\psi_{3}(x, y, t)\right)$.

Como $\psi$ é um difeomorfismo local, segue de (1) e (2) que $\frac{\partial \psi_{3}}{\partial t}(0) \neq 0$ e portanto $K$ é um difeomorfismo local.

Segue também de (1), (2) e (3) que

(1') $\varphi_{32}(x, 0)=0$.

(2') $\psi_{3}(x, y, 0)=0$.

(3') $K\left(x, y, f_{13}(x, y)\right)=\left(\varphi_{3}(x, y), \bar{f}_{13}\left(\varphi_{3}(x, y)\right)\right)$.

Portanto $f_{13}$ é $\mathcal{K}^{\delta}$-equivalente a $\vec{f}_{13}$.

Reciprocamente, suponhamos $f_{13} \mathcal{K}^{\delta}$-equivalente a $\bar{f}_{13}$. Então existe um germe de difeomorfismo

$$
\begin{aligned}
K:\left(\mathbb{C}^{2} \times \mathbb{C},(0,0)\right) & \rightarrow\left(\mathbb{C}^{2} \times \mathbb{C},(0,0)\right) \\
K(x, y, t) & \mapsto(k(x, y), \theta(x, y, t))
\end{aligned}
$$

satisfazendo

(a) $k:\left(\mathbb{C}^{2}, 0\right) \rightarrow\left(\mathbb{C}^{2}, 0\right), k(x, y)=\left(k_{1}(x, y), k_{2}(x, y)\right)$, é um germe de difeomorfismo tal que $k_{2}(x, 0)=0$.

(b) $\theta(x, y, 0)=0$

(c) $K\left(x, y, f_{13}(x, y)\right)=\left(k(x, y), \bar{f}_{13}(k(x, y))\right)$.

É fácil ver que

(a') $K \circ f_{1} \circ k^{-1}=\bar{f}_{1}$.

(b) $K \circ f_{2} \circ \phi^{-1}=\bar{f}_{2}$, onde $\phi(x, y)=\left(k_{1}(x, 0), \theta(x, 0, y)\right)$. 
(b') $K \circ f_{3} \circ k^{-1}=\bar{f}_{3}$.

Portanto $f$ é $\mathcal{A}$-equivalente a $\bar{f}$.

(ii) É imediato.

Corolário 2.4.3 Com as mesmas hipóteses de 2.4.2, $N \mathcal{A}_{e} f$ é isomorfo a $N \mathcal{K}_{e}^{\delta} f_{13}$

Corolário 2.4.4 Um trigerme simples de $\mathbb{C}^{2}$ em $\mathbb{C}^{3}$ cujos ramos são imersões que não são duas a duas tangentes, é A-equivalente a um dos seguintes:
(a) $\quad\left[S_{k}\right]:\left\{\begin{array}{l}(x, y) \mapsto(x, y, 0) \\ (x, y) \mapsto(x, 0, y) \\ (x, y) \mapsto\left(x, y, y+x^{k+1}\right)\end{array} \quad(k \geq 0)\right.$
(b) $\quad\left[B_{k}\right]:\left\{\begin{array}{l}(x, y) \mapsto(x, y, 0) \\ (x, y) \mapsto(x, 0, y) \\ (x, y) \mapsto\left(x, y, x^{2}+y^{k}\right)\end{array} \quad(k \geq 2)\right.$
(c) $\quad\left[C_{k}\right]:\left\{\begin{aligned}(x, y) & \mapsto(x, y, 0) \\ (x, y) & \mapsto(x, 0, y) \\ (x, y) & \mapsto\left(x, y, x y+x^{k}\right)\end{aligned} \quad(k \geq 3)\right.$
(d) $\quad\left[F_{4}\right]:\left\{\begin{aligned}(x, y) & \mapsto(x, y, 0) \\ (x, y) & \mapsto(x, 0, y) \\ (x, y) & \mapsto\left(x, y, x^{3}+y^{2}\right)\end{aligned}\right.$

Prova Segue do Lema 2.4.1 e da Proposição 2.4.2 que, neste caso, a classi- 
ficação é dada pela classificação de germes em $\mathcal{O}_{2}$ segundo a $\mathcal{K}^{\delta}$ equivalência, ver [1] e [26].

Com o seguinte resultado completamos a classificação dos trigermes simples de $\mathbb{C}^{2}$ em $\mathbb{C}^{3}$.

Proposição 2.4.5 Um trigerme simples de $\mathbb{C}^{2}$ em $\mathbb{C}^{3}$ cujos ramos são imersões duas a duas tangentes, não é simples.

Prova Suponhamos primeiramente que as imersões têm contato $A_{1}$.

Suponhamos, sem perda de generalidade, que uma das imersões seja a canônica. Então no plano $Z=0$ temos 4 retas (cada par dado pelo contato de uma imersão com a canônica) e seu cross-ratio é um invariante por difeomorfismos na fonte e na meta. Portanto o trigerme não pode ser simples.

O caso geral é adjacente ao discutido acima e portanto nāo é simples.

A classificação de quadrigermes $(|S|=4)$ é análoga a 2.3.3 onde consideramos $\mathcal{X}$ a imagem do ponto triplo ordinário:

$$
\left\{\begin{array}{l}
(x, y) \mapsto(x, y, 0) \\
(x, y) \mapsto(x, 0, y) \\
(x, y) \mapsto(0, x, y)
\end{array}\right.
$$

Portanto temos o seguinte

Teorema 2.4.6 A lista abaixo consiste nos multigermes simples de $\mathbb{C}^{2}$ em $\mathbb{C}^{3}$ segundo $\mathcal{A}$ equivalência. 
Monogerme

$f(x, y)=(x, y, 0)$

$f(x, y)=\left(x, y^{2}, x y\right)$

$f(x, y)=\left(x, y^{2}, y^{3}+x^{k+1} y\right)$

$f(x, y)=\left(x, y^{2}, x^{2} y+y^{2 k+1}\right)$,

$f(x, y)=\left(x, y^{2}, x y^{3}+x^{k} y\right)$

$f(x, y)=\left(x, y^{2}, x^{3} y+y^{5}\right)$

$f(x, y)=\left(x, y^{3}, x y+y^{3 k-1}\right)$,

Bigerme

$f:\left\{\begin{aligned} &(x, y) \mapsto(x, y, 0) \\ &(x, y) \mapsto(x, 0, y)\end{aligned}\right.$

$f:\left\{\begin{array}{l}(x, y) \mapsto(x, y, 0) \\ (x, y) \mapsto\left(x, y, x^{2}+y^{k+1}\right)\end{array}\right.$

$f:\left\{\begin{array}{rl}(x, y) & \mapsto(x, y, 0) \\ (x, y) & \mapsto\left(x, y, x^{2} y+y^{k-1}\right)\end{array} \quad k \geq 4\right.$
$\mathcal{A}_{e}-$ codim

0

0

Cross $-\operatorname{cap}\left(S_{0}\right)$

$k \geq 1$

$k$

$S_{k}$

$B_{k}$

$C_{k}$

4

$F_{4}$

$H_{k}$

$k \geq 2$

$k$

$\mathcal{A}_{e}-$ codim

Nome

Ponto Duplo

Ordinário

$k$

$\left[A_{k}\right]$

$k$

$\left[D_{k}\right]$ 


$$
\begin{aligned}
& f:\left\{\begin{aligned}
(x, y) & \mapsto(x, y, 0) \\
(x, y) & \mapsto\left(x, y, x^{3}+y^{4}\right)
\end{aligned}\right. \\
& f:\left\{\begin{aligned}
(x, y) & \mapsto(x, y, 0) \\
(x, y) & \mapsto\left(x, y, x^{3}+x y^{3}\right)
\end{aligned}\right. \\
& f:\left\{\begin{aligned}
(x, y) & \mapsto(x, y, 0) \\
(x, y) & \mapsto\left(x, y, x^{3}+y^{5}\right)
\end{aligned}\right. \\
& f:\left\{\begin{aligned}
(x, y) & \mapsto\left(x, y^{2}, x y\right) \\
(x, y) & \mapsto\left(x^{k}, x, y\right)
\end{aligned}\right. \\
& f:\left\{\begin{aligned}
(x, y) & \mapsto\left(x, y^{2}, x y\right) \\
(x, y) & \mapsto\left(x, x^{k+1}, y\right)
\end{aligned}\right. \\
& f:\left\{\begin{aligned}
(x, y) & \mapsto\left(x, y^{2}, x y\right) \\
(x, y) & \mapsto\left(x, y, y^{k+1}\right)
\end{aligned}\right. \\
& f:\left\{\begin{array}{rl}
(x, y) & \mapsto\left(x, y^{2}, y^{3}+x^{k+1} y\right) \\
(x, y) & \mapsto(0, x, y)
\end{array} \quad k+1\right. \\
& \text { Trigerme } \\
& f:\left\{\begin{aligned}
(x, y) & \mapsto(x, y, 0) \\
(x, y) & \mapsto(x, 0, y) \\
(x, y) & \mapsto(0, x, y)
\end{aligned}\right. \\
& k+2 \quad\left[S_{0} A_{k}\right](k+1) \\
& \mathcal{A}_{e} \rightarrow \text { codim } \\
& \text { Nome } \\
& {\left[S_{k} A_{0}\right]}
\end{aligned}
$$




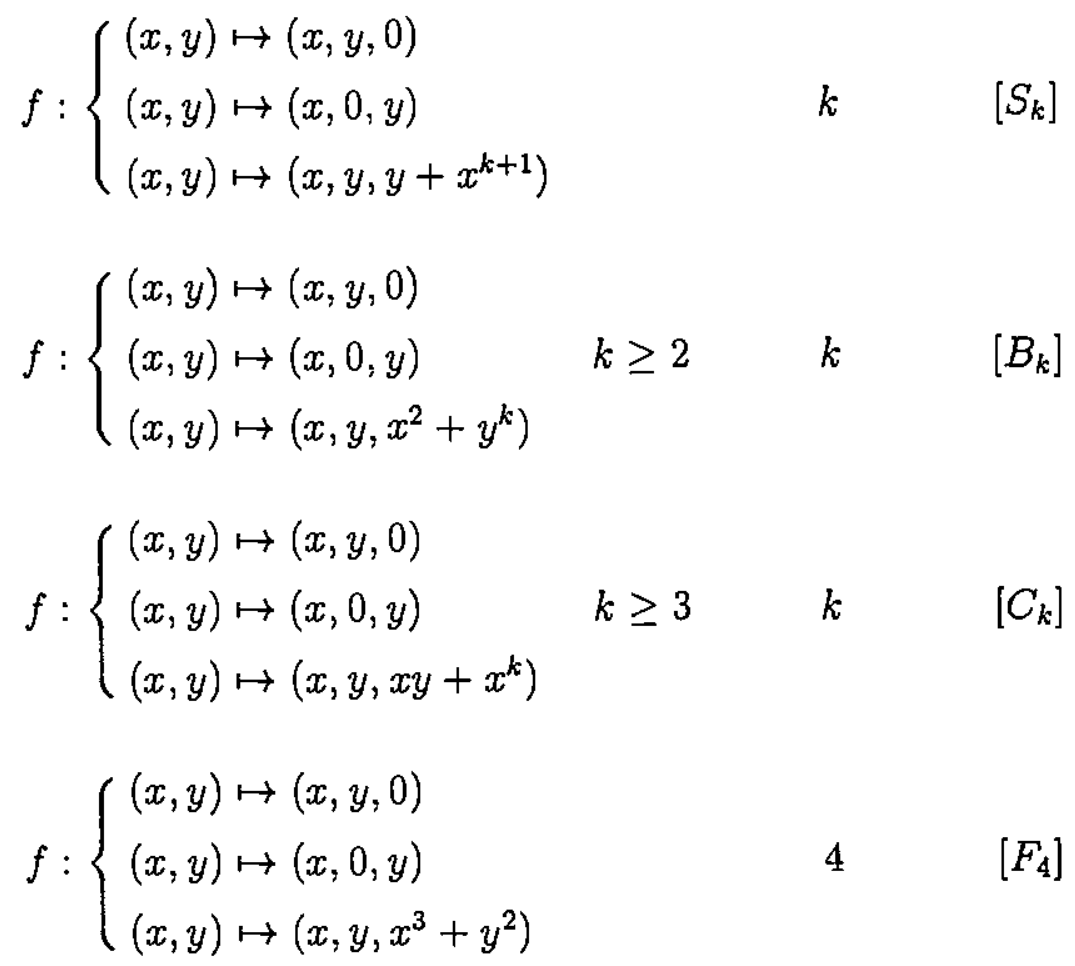

$$
f:\left\{\begin{aligned}
(x, y) & \mapsto(x, y, 0) \\
(x, y) & \mapsto(x, 0, y) \\
(x, y) & \mapsto(0, x, y) \\
(x, y) & \mapsto\left(x, y, y+x^{k}\right)
\end{aligned}\right.
$$

$* k \geq 1$ exceto nos casos explicitados.

* Em parênteses temos o número de pontos triplos na imagem de uma perturbação estável (ver o capítulo 4 desta tese). 


\subsection{Adjacências}

\subsubsection{Adjacências de bigermes}
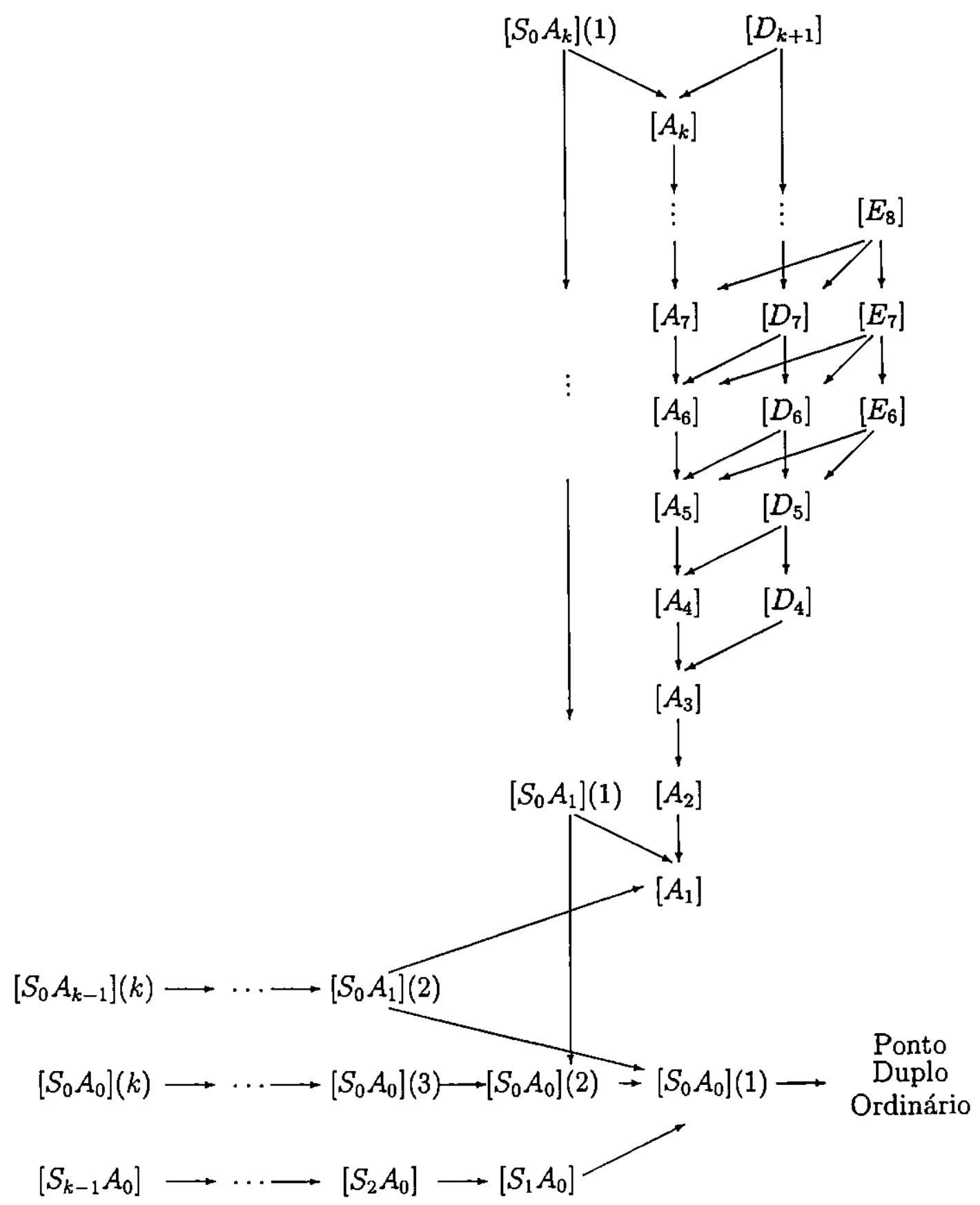


\subsubsection{Adjacências de trigermes}

$$
\begin{aligned}
& {\left[F_{4}\right]} \\
& {\left[C_{k}\right] \longrightarrow \cdots \longrightarrow\left[C_{4}\right] \longrightarrow\left[C_{3}\right]} \\
& {\left[B_{k}\right] \longrightarrow \cdots \longrightarrow\left[B_{4}\right] \longrightarrow\left[B_{3}\right] \longrightarrow\left[B_{2}\right]} \\
& {\left[S_{k}\right] \longrightarrow \cdots \longrightarrow\left[S_{4}\right] \longrightarrow\left[S_{3}\right] \longrightarrow\left[S_{2}\right] \longrightarrow\left[S_{1}\right] \longrightarrow \begin{array}{c}
\text { Ponto } \\
\text { Triplo } \\
\text { Ordinário }
\end{array}}
\end{aligned}
$$




\section{Capítulo 3}

\section{A topologia do complementar do conjunto de bifurcação}

\subsection{Introdução}

Em seus trabalhos sobre classificação de germes, V.I.Arnold mostra que, em muitos casos, o complementar do conjunto de bifurcação é um espaço de Eilenberg-MacLane $K(\pi, 1)$.

Em [3] V.V.Goryunov mostra que no caso da classificação de monogermes simples de $\mathbb{C}^{2}$ em $\mathbb{C}^{3}$ dada por D.Mond, este mesmo resultado vale, ou seja, o complementar do conjunto de bifurcação é um espaço do tipo $K(\pi, 1)$.

Neste capítulo estendemos o resultado de Goryunov para os multigermes simples de $\mathbb{C}^{2}$ em $\mathbb{C}^{3}$ obtidos no capítulo 2. Como a classificação de alguns multigermes recai sobre os resultados de Arnold, veremos que o mesmo acontece aqui, ou seja, alguns conjuntos de bifurcação são análogos aos de Arnold e portanto já sabemos serem do tipo $K(\pi, 1)$. 


\subsection{O conjunto de bifurcação}

O conjunto de bifurcação $\Sigma$ de um germe de $\left(\mathbb{R}^{2}, S\right)$ em $\left(\mathbb{R}^{3}, 0\right)$ (ver por exemplo [3]) é o conjunto dos valores do parâmetro $\lambda$ de uma deformação $\mathcal{A}_{e^{-}}$ miniversal para os quais as imagens das correspondentes aplicaçōes têm singularidades mais complicadas que em posição geral, isto é, são nāo estáveis. As singularidades de uma imagem em posição geral são o cross-cap e intersecçōes transversais de 2 ou 3 planos (pontos duplos e triplos). Como vimos no capítulo 2 , existem 5 singularidades não estáveis que ocorrem estavelmente em famílias a 1-parâmetro de aplicações $\mathbb{R}^{2} \rightarrow \mathbb{R}^{3}$. Elas correspondem a 5 componentes (no caso mais geral) de $\Sigma$. A seguir descrevemos estas componentes que denotamos por $\Sigma_{1}, \ldots, \Sigma_{5}$.

$\Sigma_{1}$ : fusão/nascimento de 2 cross-caps. Este fenômeno ocorre com os monogermes, quando são adjacentes a $S_{1}$.

$\Sigma_{2}$ : contato não transversal de dois planos imersos. Ocorre quando dois ramos não singulares se interceptam e os planos tangentes no ponto de intersecção coincidem..

$\Sigma_{3}$ : intersecção de cross-cap e imersão. Ocorre quando um ramo não singular intercepta um cross-cap transversalmente. Como se trata de codimensāo 1, o plano imerso deve interceptar transversalmente a curva de pontos duplos do cross-cap.

$\Sigma_{4}$ : ponto triplo não transversal. Ocorre quando dois ramos não singulares se interceptam transversalmente e um terceiro ramo não singular intercepta esta curva de pontos duplos tangencialmente.

$\Sigma_{5}$ : ponto quadruplo transversal. 
Definição 3.2.1 Um espaço topológico do tipo $K(\pi, n)$ (ou um espaço de Eilenberg-MacLane), onde $\pi$ é um grupo e $n$ é um inteiro maior ou igual a 1, é um espaço $Y$ conexo por caminhos que satisfaz $\pi_{q}\left(Y, y_{0}\right)=0$ para $q \neq n$ e $\pi_{n}\left(Y, y_{0}\right)$ é isomorfo $a \pi$.

Teorema 3.2.2 O complementar do conjunto de bifurcação de um multigerme simples de $\mathbb{C}^{2}$ em $\mathbb{C}^{3}$ é um espaço do tipo $K(\pi, 1)$.

Prova O resultado já é conhecido para os monogermes [3].

Sabe-se que, no caso complexo, o complementar do conjunto de bifurcação é um espaço conexo.

Para bigermes de imersões a única componente de $\Sigma$ não vazia é $\Sigma_{2}$. Neste caso $\Sigma$ coincide com o conjunto de bifurcação da função de contato e portanto seu complementar é um espaço do tipo $K(\pi, 1)$ [4].

De fato, consideremos o caso $\left[A_{k}\right]$. A função de contato é dada por $\varphi(x, y)=x^{2}+y^{k+1}$. Seja

$$
f_{\lambda}:\left\{\begin{aligned}
(x, y) & \mapsto(x, y, 0) \\
(x, y) & \mapsto\left(x, y, \varphi_{\lambda}(x, y)\right)
\end{aligned}\right.
$$

um desdobramento $\mathcal{A}_{e}$-miniversal de $\left[A_{k}\right]$, onde $\varphi_{\lambda}(x, y)=x^{2}+y^{k+1}+$ $\lambda_{k-1} y^{k-1}+\cdots+\lambda_{1} y+\lambda_{0}$.

Seja $U_{i}$ uma vizinhança no domínio de $f_{\lambda}^{(i)}$. Como ambos os ramos são imersões, pontos duplos somente ocorrem quando $f_{\lambda}^{(1)}$ e $f_{\lambda}^{(2)}$ se interceptam:

$$
D^{2}\left(f_{\lambda}\right) \cap\left(U_{1} \times U_{2}\right) \cong\left\{(x, y) / \varphi_{\lambda}(x, y)=0\right\}
$$

Como

$$
\left(d f_{\lambda}^{(1)}\right)=\left(\begin{array}{cc}
1 & 0 \\
0 & 1 \\
0 & 0
\end{array}\right) \quad\left(d f_{\lambda}^{(2)}\right)=\left(\begin{array}{cc}
1 & 0 \\
0 & 1 \\
2 x & \frac{\partial \varphi_{\lambda}}{\partial y}
\end{array}\right)
$$


a intersecção de $f_{\lambda}^{(1)}$ e $f_{\lambda}^{(2)}$ não é transversal quando $x=0$ e $\frac{\partial \varphi_{\lambda}}{\partial y}=0$.

Assim, $\Sigma$ é dado por

$$
\left\{\begin{array}{l}
\varphi_{\lambda}(0, y)=0 \\
\frac{\partial \varphi_{\lambda}}{\partial y}(0, y)=0
\end{array}\right.
$$

No caso dos trigermes, o conjunto de bifurcação coincide com o conjunto de bifurcação da função do bordo.

De fato, consideremos o caso $\left[B_{k}\right]$. Seja

$$
g_{\lambda}:\left\{\begin{aligned}
(x, y) & \mapsto(x, y, 0) \\
(x, y) & \mapsto(x, 0, y) \\
(x, y) & \mapsto\left(x, y, p_{\lambda}(x, y)\right)
\end{aligned}\right.
$$

um desdobramento $\mathcal{A}_{e^{-}}$miniversal de $\left[B_{k}\right]$, onde $p_{\lambda}(x, y)=x^{2}+y^{k}+\lambda_{k-1} y^{k-1}+$ $\cdots+\lambda_{1} y+\lambda_{0}$.

Como todos os ramos são imersões temos que $\Sigma_{1}$ e $\Sigma_{3}$ são vazios.

$\Sigma_{2}$ : Seja $U_{i}$ uma vizinhança no domínio de $g_{\lambda}^{(i)}$. Pontos duplos não transversais são provenientes de $g_{\lambda}^{(1)}$ e $g_{\lambda}^{(3)}$ :

$$
\begin{gathered}
D^{2}\left(g_{\lambda}\right) \cap\left(U_{1} \times U_{3}\right) \cong\left\{(x, y) / p_{\lambda}(x, y)=0\right\} \\
\left(d g_{\lambda}^{(1)}\right)=\left(\begin{array}{ll}
1 & 0 \\
0 & 1 \\
0 & 0
\end{array}\right) \quad\left(d g_{\lambda}^{(3)}\right)=\left(\begin{array}{cc}
1 & 0 \\
0 & 1 \\
2 x & \frac{\partial p_{\lambda}}{\partial y}
\end{array}\right)
\end{gathered}
$$

Logo $g_{\lambda}^{(1)}$ e $g_{\lambda}^{(3)}$ se interceptam tangencialmente quando

$$
\left\{\begin{array}{l}
p_{\lambda}(0, y)=0 \\
\frac{\partial p_{\lambda}}{\partial y}(0, y)=0
\end{array}\right.
$$

$\Sigma_{4}:$ Temos que

$$
D^{2}\left(g_{\lambda}\right) \cap\left(U_{1} \times U_{2}\right) \cong\{(x, 0)\}
$$




$$
D^{3}\left(g_{\lambda}\right) \cap\left(U_{1} \times U_{2} \times U_{3}\right) \cong\left\{(x, 0) / p_{\lambda}(x, 0)=0\right\}
$$

Também, $(1,0,0)$ é um vetor tangente à imagem de $D^{2}\left(g_{\lambda}\right) \cap\left(U_{1} \times U_{2}\right)$ e $\left(-2 x,-\frac{\partial p_{\lambda}}{\partial y}, 1\right)$ é um vetor normal ao plano tangente à imagem de $g_{\lambda}^{(3)}$. Assim, $g_{\lambda}^{(3)}$ intercepta a curva de pontos duplos $D^{2}\left(g_{\lambda}\right) \cap\left(U_{1} \times U_{2}\right)$ tangencialmente quando $x=0$. Logo temos $x=0$ e $p_{\lambda}(x, 0)=0$. Portanto $\Sigma_{4}=\left\{\lambda_{0}=0\right\}$.

Portanto $\Sigma=\Sigma_{2} \cup \Sigma_{4}=\left\{\mathcal{D}\left(p_{\lambda}(0, y)\right)=0\right\} \cup\left\{\lambda_{0}=0\right\}$, onde $\mathcal{D}\left(p_{\lambda}(0, y)\right)$ é o discriminante de $p_{\lambda}(0, y)$.

Logo o conjunto de bifurcação de $\left[B_{k}\right]$ coincide com o conjunto de bifurcação de $B_{k}$ e portanto seu complementar é um espaço do tipo $K(\pi, 1)$ [1].

Resta portanto mostrar para os bigermes $\left[S_{0} A_{0}\right](k),\left[S_{0} A_{k}\right](1),\left[S_{0} A_{k}\right](k+$ 1), $\left[S_{k} A_{0}\right]$ e o quadrigerme $Q_{k+1}$. Mostraremos estes casos nos lemas seguintes.

Lema 3.2.3 (i) Um desdobramento $\mathcal{A}_{\boldsymbol{e}}$-versal de $\left[S_{0} A_{0}\right](k)$ é dado por

$$
f_{\lambda}:\left\{\begin{aligned}
(x, y) & \mapsto\left(x, y^{2}, x y\right) \\
(x, y) & \mapsto\left(p_{\lambda}(x), x, y\right)
\end{aligned}\right.
$$

onde $p_{\lambda}(x)=x^{k}+\lambda_{k-1} x^{k-1}+\cdots+\lambda_{1} x+\lambda_{0}$.

(ii) Um desdobramento $\mathcal{A}_{e}$-versal de $\left[S_{0} A_{k}\right](1)$ é dado por

$$
f_{\lambda}:\left\{\begin{aligned}
(x, y) & \mapsto\left(x, y^{2}, x y\right) \\
(x, y) & \mapsto\left(x, p_{\lambda}(x), y\right)
\end{aligned}\right.
$$

onde $p_{\lambda}(x)=x^{k+1}+\lambda_{k} x^{k}+\lambda_{k-1} x^{k-1}+\cdots+\lambda_{1} x+\lambda_{0}$.

(iii) Em ambos os casos o conjunto de bifurcação tem 2 componentes: $\left\{\lambda_{0}=\right.$ $0\}$ e a outra dada pelo discriminante de $p_{\lambda}$. Portanto o complementar de $\Sigma$ é um espaço do tipo $K(\pi, 1)$. 
Lema 3.2.4 (i) Um desdobramento $\mathcal{A}_{e}$-versal de $\left[S_{0} A_{k}\right](k+1)$ é dado por

$$
f_{\lambda}:\left\{\begin{aligned}
(x, y) & \mapsto\left(x, y^{2}+a y, x y\right) \\
(x, y) & \mapsto\left(x, y, p_{\lambda}(y)\right)
\end{aligned}\right.
$$

onde $p_{\lambda}(y)=y^{k+1}+\lambda_{k} y^{k}+\lambda_{k-1} y^{k-1}+\cdots+\lambda_{1} y+\lambda_{0}$.

(ii) O conjunto de bifurcação tem 3 componentes: $\left\{\lambda_{0}=0\right\},\left\{p_{\lambda}\left(-a^{2} / 4\right)=0\right\}$ $e\left\{\mathcal{D}\left(p_{\lambda}\left(y^{2}+a y\right)\right)=0\right\}$.

(iii) $\mathbb{C}^{k+2} \backslash \Sigma$ é um espaço do tipo $K(\pi, 1)$.

Lema 3.2.5 (i) Um desdobramento $\mathcal{A}_{e}$-versal de $\left[S_{k} A_{0}\right]$ é dado por

$$
f_{\lambda}:\left\{\begin{aligned}
(x, y) & \mapsto\left(x, y^{2}, y p_{\lambda}\left(x, y^{2}\right)\right) \\
(x, y) & \mapsto(0, x, y)
\end{aligned}\right.
$$

onde $p_{\lambda}(x, y)=y+x^{k+1}+\lambda_{k} x^{k}+\lambda_{k-1} x^{k-1}+\cdots+\lambda_{1} x+\lambda_{0}$.

(ii) O conjunto de bifurcação tem 2 componentes: $\left\{\lambda_{0}=0\right\}$ e $\left\{\mathcal{D}\left(p_{\lambda}(x, 0)\right)=\right.$ $0\}$. Portanto $\mathbb{C}^{k+1} \backslash \Sigma$ é um espaço do tipo $K(\pi, 1)$.

Lema 3.2.6 Um desdobramento $\mathcal{A}_{e}$-versal de $Q_{k+1}$ é dado por

$$
f_{\lambda}:\left\{\begin{aligned}
(x, y) & \mapsto(x, y, 0) \\
(x, y) & \mapsto(x, 0, y) \\
(x, y) & \mapsto(0, x, y) \\
(x, y) & \mapsto\left(x, y, p_{\lambda}(x, y)\right)
\end{aligned}\right.
$$

onde $p_{\lambda}(x, y)=y+x^{k+1}+\lambda_{k} x^{k}+\cdots+\lambda_{1} x+\lambda_{0}$.

(ii) $O$ conjunto de bifurcação tem 2 componentes: $\left\{\lambda_{0}=0\right\}$ e $\left\{\mathcal{D}\left(p_{\lambda}(x, 0)\right)=\right.$ $0\}$. Portanto $\mathbb{C}^{k+1} \backslash \Sigma$ é um espaço do tipo $K(\pi, 1)$.

Faremos somente a demonstração do Lema 3.2.4. Os demais são análogos. Prova(Lema 3.2.4) (ii) Temos que $\Sigma_{1}$ e $\Sigma_{5}$ são vazios. 
$\Sigma_{2}:$

$$
\begin{gathered}
D^{2}\left(f_{\lambda}\right) \cap\left(U_{1} \times U_{2}\right) \cong\left\{\left(x, y_{1}, y_{2}\right) / y_{2}=y_{1}^{2}+a y_{1}, p_{\lambda}\left(y_{2}\right)-x y_{1}=0\right\} \\
\left(d f_{\lambda}^{(1)}\right)_{\left(x, y_{1}\right)}=\left(\begin{array}{cc}
1 & 0 \\
0 & 2 y_{1}+a \\
y_{1} & x
\end{array}\right) \quad\left(d f_{\lambda}^{(2)}\right)_{\left(x, y_{2}\right)}=\left(\begin{array}{cc}
1 & 0 \\
0 & 1 \\
0 & \frac{d p_{\lambda}}{d y}\left(y_{2}\right)
\end{array}\right)
\end{gathered}
$$

Um vetor normal ao plano tangente à imagem de $f_{\lambda}^{(1)}$ é $\left(-y_{1}\left(2 y_{1}+a\right),-x, 2 y_{1}+\right.$ a) e um vetor normal ao plano tangente à imagem de $f_{\lambda}^{(2)}$ é $\left(0,-\frac{d p_{\lambda}}{d y}\left(y_{2}\right), 1\right)$. Logo $f_{\lambda}^{(1)}$ e $f_{\lambda}^{(2)}$ se interceptam tangencialmente quando $y_{1}=0$. Portanto pontos duplos não transversais ocorrem quando $p_{\lambda}(0)=0$, ou seja, $\lambda_{0}=0$. Temos entāo $\Sigma_{2}=\left\{\lambda_{0}=0\right\}$.

$\Sigma_{3}$ : um ponto $\left(x_{0}, y_{0}\right)$ é um cross-cap de $f_{\lambda}^{(1)}$ se $2 y_{0}+a=0$ e $x_{0}=0$ (ver [15, pag 179]). O ramo imersivo deve ser transversal a curva de pontos duplos do cross-cap:

$$
\begin{aligned}
D^{2}\left(f_{\lambda}\right) \cap\left(U_{1} \times U_{1}\right) & \cong\left\{\left(x_{1}, y_{1}, x_{2}, y_{2}\right) / f_{\lambda}^{(1)}\left(x_{1}, y_{1}\right)=f_{\lambda}^{(1)}\left(x_{2}, y_{2}\right)\right\} \\
& =\left\{\left(0, y_{1}, 0, y_{2}\right) / y_{1}+y_{2}+a=0\right\}
\end{aligned}
$$

Assim, $f_{\lambda}^{(1)}$ intercepta $f_{\lambda}^{(2)}$ e $f_{\lambda}^{(1)}$ é um cross-cap no ponto de interseç̧ão se $2 y+a=0$ e $p_{\lambda}\left(y^{2}+a y\right)=0$. Portanto $\Sigma_{3}$ é dada por:

$$
\left\{\begin{array}{l}
2 y+a=0 \\
p_{\lambda}\left(y^{2}+a y\right)=0
\end{array}\right.
$$

$\Sigma_{4}$ : ponto triplo não transversal.

$$
D^{3}\left(f_{\lambda}\right) \cap\left(U_{1} \times U_{1} \times U_{2}\right) \cong\left\{(0, y) / p_{\lambda}\left(y^{2}+a y\right)=0\right\}
$$

Um vetor tangente à imagem de $D^{2}\left(f_{\lambda}\right) \cap\left(U_{1} \times U_{1}\right)$ é dado por $(0,2 y+a, 0)$ e um vetor normal ao plano tangente à imagem de $f_{\lambda}^{(2)}$ é $\left(0,-\frac{d p_{\lambda}}{d y}\left(y^{2}+a y\right), 1\right)$. 
Assim a condição de não transversalidade implica que $(2 y+a) \cdot \frac{d p_{\lambda}}{d y}\left(y^{2}+a y\right)=$ 0. Portanto $\Sigma_{4}=\left\{\mathcal{D}\left(p_{\lambda}\left(y^{2}+a y\right)\right)=0\right\}$.

(iii) Seja $P$ o espaço dos polinômios de grau $k+1$ com coeficiente dominante 1.

Consideremos $\varphi: \mathbb{C}^{k+2} \rightarrow \mathbb{C} \times P$ dada por

$$
\varphi\left(z_{0}, z_{1}, \ldots, z_{k+1}\right)=\left(z_{0},\left(z-z_{1}\right) \cdots\left(z-z_{k+1}\right)\right)
$$

Temos que

$$
\begin{aligned}
& \varphi^{-1}(\mathbb{C} \times P \backslash \Sigma)=\left\{\left(z_{0}, z_{1}, \ldots, z_{k+1}\right) / z_{i} \neq z_{j} \quad \forall i \neq j, \quad i, j=1, \ldots, k+1,\right. \\
& \left.z_{i} \neq 0, z_{i} \neq-z_{0}^{2} / 4, i=1, \ldots, k+1\right\}
\end{aligned}
$$

Seja $Y=\mathbb{C} \times P \backslash \Sigma$ e $\tilde{Y}=\varphi^{-1}(\mathbb{C} \times P \backslash \Sigma)$.

Então $\left.\varphi\right|_{\bar{Y}}: \tilde{Y} \rightarrow Y$ é uma aplicação de recobrimento e portanto $\pi_{n}(\tilde{Y}) \cong$ $\pi_{n}(Y)$ para $n \geq 2$.

Seja $\mathcal{B}_{k+1}=\left\{\left(z_{1}, \ldots, z_{k+1}\right) / z_{i} \neq 0, z_{i} \neq z_{j} \forall i \neq j\right\}$ e $\psi: \tilde{Y} \rightarrow \mathcal{B}_{k+1}$ dada por $\psi\left(z_{0}, z_{1}, \ldots, z_{k+1}\right)=\left(z_{1}, \ldots, z_{k+1}\right)$.

Então $\psi$ é uma fibração cujas fibras são isomorfas a $\mathbb{C}$ menos $2(k+1)$ pontos distintos. A fibra é portanto um espaço do tipo $K(\pi, 1)$.

Como $\mathcal{B}_{k+1}$ é um espaço do tipo $K(\pi, 1)$ (ver [30]), segue da sequência de homotopia de uma fibração [34] que $\tilde{Y}$ é um espaço do tipo $K(\pi, 1)$.

Portanto $\mathbb{C}^{k+2} \backslash \Sigma$ é um espaço do tipo $K(\pi, 1)$. 


\section{Capítulo 4}

\section{A topologia da imagem de uma perturbação estável}

\subsection{Introdução}

Dado um multigerme $f$ de $\mathbb{C}^{n}$ em $\mathbb{C}^{n+1}$, finitamente determinado, $(n, n+1)$ no domínio das boas dimensões segundo Mather, podemos construir uma fibração definida na imagem de um desdobramento versal de $f$ com valores no complementar do conjunto de bifurcação. A fibra tem o tipo de homotopia de um bouquet de n-esferas. O número de esferas no bouquet é chamado número de Milnor da imagem de $f$.

Os monogermes $S_{k}, B_{k}, C_{k}$ e $F_{4}$, isto é, da forma $\left(x, y^{2}, y p\left(x, y^{2}\right)\right)$, e os trigermes $\left[S_{k}\right],\left[B_{k}\right],\left[C_{k}\right]$ e $\left[F_{4}\right]$, possuem o mesmo conjunto de bifurcação, a saber o discriminante da correspondente função do bordo (ver [3, pag.62] e a prova do Teorema 3.2.2). Logo surge a questão: qual a relação entre as fibrações acima descritas? Neste capítulo mostraremos que as fibras são homotópicas. Para isto usaremos o método desenvolvido por D.Mond e 
V.V.Goryunov [16]. Na seção 4.2 trataremos deste método, com uma breve introdução sobre sequências espectrais, cuja referência é [34].

Em [27] D.Mond define os invariantes $C, T$ e $\mu\left(D^{2}(f) / \mathbb{Z}_{2}\right)$ para monogermes de $\mathbb{C}^{2}$ em $\mathbb{C}^{3}$, que são o número de cross-caps e pontos triplos presentes na imagem de uma perturbação estável do germe e o número de Milnor da singularidade isolada $D^{2}(f) / \mathbb{Z}_{2}$ respectivamente. Na seção 4.4 discutiremos esta questão dos invariantes para multigermes.

\subsection{Método Goryunov-Mond}

Um módulo bigraduado $E$ sobre um anel $R$ é uma coleção de $R$-módulos $E_{s, t}$ para todo par de inteiros $s$ and $t$. Uma diferencial $d: E \rightarrow E$ de bigrau $(-r, r-1)$ é uma coleção de homomorfismos $d: E_{s, t} \rightarrow E_{s-r, t+r-1}$, para todo $s$ e $t$, tais que $d^{2}=0$. O módulo de homologia $H(E)$ é o módulo bigraduado dado por

$$
H_{s, t}(E)=\left[k e r\left(d: E_{s, t} \rightarrow E_{s-r, t+r-1}\right)\right] / d\left(E_{s+r, t-r+1}\right)
$$

Uma sequência espectral $E$ é uma sequência $\left\{E^{r}, d^{r}\right\}, r \geq 1$, tal que

(a) $E^{r}$ é um módulo bigraduado e $d^{r}$ é uma diferencial de bigrau $(-r, r-1)$ em $E^{r}$.

(b) Existe um isomorfismo $H\left(E^{r}\right) \cong E^{r+1}$.

Para definir o limite de uma sequência espectral, identificamos $E^{r+1}$ com $H\left(E^{r}\right)$. Seja $Z^{1}$ o módulo bigraduado $Z_{s, t}^{1}=k e r\left(d: E_{s, t}^{1} \rightarrow E_{s-1, t}^{1}\right)$ e $B^{1}$ o módulo bigraduado $B_{s, t}^{1}=d^{1}\left(E_{s+1, t}^{1}\right)$. Então $B^{1} \subset Z^{1}$ e $E^{2}=Z^{1} / B^{1}$. Seja $Z\left(E^{2}\right)$ o módulo bigraduado $Z\left(E^{2}\right)_{s, t}=\operatorname{ker}\left(d^{2}: E_{s, t}^{2} \rightarrow E_{s-2, t+1}^{2}\right)$ e $B\left(E^{2}\right) \circ$ módulo bigraduado $B\left(E^{2}\right)_{s, t}=d^{2}\left(E_{s+2, t-1}^{2}\right)$. Pelo Teorema do isomorfismo de 
Noether, existem submódulos bigraduados $Z^{2}$ e $B^{2}$ de $Z^{1}$ contendo $B^{1}$ tais que $Z\left(E^{2}\right)_{s, t}=Z_{s, t}^{2} / B_{s, t}^{1}$ e $B\left(E^{2}\right)_{s, t}=B_{s, t}^{2} / B_{s, t}^{1}$ para todo $s$ e $t$. Segue que $B^{2} \subset Z^{2} \mathrm{e}$

$$
B^{1} \subset B^{2} \subset Z^{2} \subset Z^{1}
$$

Prosseguindo por indução, obtemos submódulos

$$
B^{1} \subset B^{2} \subset \ldots \subset B^{r} \subset \ldots \subset Z^{r} \subset \ldots \subset Z^{2} \subset Z^{1}
$$

tais que $E^{r+1}=Z^{r} / B^{r}$. Definimos os módulos bigraduados $Z^{\infty}=\cap_{r} Z^{r}$, $B^{\infty}=\cup_{r} B^{r}$ e $E^{\infty}=Z^{\infty} / B^{\infty}$. O módulo bigraduado $E^{\infty}$ é chamado limite da sequência espectral $E$, e os termos $E^{r}$ da sequência espectral são aproximaçōes sucessivas de $E^{\infty}$.

Dizemos que a sequência espectral $E$ converge se para todo $s$ e $t$ existe um inteiro $r(s, t) \geq 1$ tal que para $r \geq r(s, t), d^{r}: E_{s, t}^{r} \rightarrow E_{s-r, t+r-1}^{r}$ é trivial. Então $E_{s, t}^{r+1}$ é isomorfo a um quociente de $E_{s, t}^{r}$ e $E_{s, t}^{\infty}$ é isomorfo ao limite da sequência

$$
E_{s, t}^{r(s, t)} \rightarrow E_{s, t}^{r(s, t)+1} \rightarrow \ldots
$$

Dizemos que uma sequência espectral colapsa no $N^{o}$ termo se $d^{r}=0$ para $r \geq N$. Portanto $E_{s, t}^{r}=E_{s, t}^{N}$ e $E_{s, t}^{\infty}=E_{s, t}^{N}$.

Sejam $X$ e $Y$ espaços topológicos e $f: X \rightarrow Y$ contínua. Seja $D^{k}(f)$ o conjunto dos pontos $k$-múltiplos de $f$ :

$$
D^{k}(f)=\operatorname{fecho}\left\{\left(x_{1}, \ldots, x_{k}\right) \in X^{k} / f\left(x_{1}\right)=\ldots=f\left(x_{k}\right), x_{i} \neq x_{j} \text { se } i \neq j\right\}
$$

para $1 \leq k<\infty$.

O grupo simétrico $S_{k}$ age em $D^{k}(f)$ permutando os fatores. Portanto $S_{k}$ age em $H_{\ell}\left(D^{k}(f) ; \mathbb{Q}\right)$. Se $\sigma \in S_{k}$, denotemos por $\sigma^{*}$ sua ação em 
$H_{\ell}\left(D^{k}(f) ; \mathbb{Q}\right):$

$$
\begin{array}{rlc}
\sigma^{*}: H_{\ell}\left(D^{k}(f) ; \mathbb{Q}\right) & \rightarrow & H_{\ell}\left(D^{k}(f) ; \mathbb{Q}\right) \\
{[\alpha]} & \mapsto & {[\sigma(\alpha)]}
\end{array}
$$

A parte alternada é dada por:

$$
A l t_{k} H_{\ell}\left(D^{k}(f) ; \mathbb{Q}\right)=\left\{c \in H_{\ell}\left(D^{k}(f) ; \mathbb{Q}\right) / \forall \sigma \in S_{k}, \sigma^{*} c=\operatorname{sign}(\sigma) c\right\}
$$

Existem aplicaçōes contínuas $\varepsilon^{i, k}: D^{k}(f) \rightarrow D^{k-1}(f), \varepsilon^{i, k}\left(x_{1}, \ldots, x_{k}\right)=$ $\left(x_{1}, \ldots, \hat{x}_{i}, \ldots, x_{k}\right)$ para $i \leq k$.

Temos então $\varepsilon_{*}^{i, k}: H_{\ell}\left(D^{k}(f) ; \mathbb{Q}\right) \rightarrow H_{\ell}\left(D^{k-1}(f) ; \mathbb{Q}\right)$ para $\ell=0,1, \ldots$

Definimos

$$
\varepsilon_{*}^{k}: A l t_{k} H_{\ell}\left(D^{k}(f) ; \mathbb{Q}\right) \rightarrow A l t_{k-1} H_{\ell}\left(D^{k-1}(f) ; \mathbb{Q}\right)
$$

por

$$
\varepsilon_{*}^{k}(c)=\frac{1}{k} \sum_{i=1}^{k}(-1)^{i+1} \varepsilon_{*}^{i, k}(c)
$$

Proposição 4.2.1 $\varepsilon_{*}^{1, k}=\varepsilon_{*}^{k}$.

Seja $f:\left(\mathbb{C}^{n}, S\right) \rightarrow\left(\mathbb{C}^{n+1}, 0\right)$ um multigerme finitamente $\mathcal{A}$-determinado de coposto $\leq 1$, ou seja, cada ramo tem coposto $\leq 1,(n, n+1)$ no domínio das boas dimensões segundo Mather. Seja $F:\left(\mathbb{C}^{n} \times \mathbb{C}^{d}, S \times\{0\}\right) \rightarrow\left(\mathbb{C}^{n+1} \times \mathbb{C}^{d}\right.$, $(0,0)), F(x, t)=\left(f_{t}(x), t\right)$, um desdobramento versal de $f$ e $F: U \rightarrow V$ um representante de $F$ (usaremos a mesma letra) que é próprio, finito a um, $F(U) \subset V, F^{-1}(0) \cap U=S \times\{0\}$ e $I_{\text {rel }}(F)=\{(y, t) /$ o germe de $f_{t}$ em $f_{t}^{-1}(y) \cap U_{t}$ não é estável $\}$ é um subconjunto analítico de $V$, onde $U_{t}=\left\{x \in \mathbb{C}^{n} /(x, t) \in U\right\}$. Seja $\pi: \mathbb{C}^{n+1} \times \mathbb{C}^{d} \rightarrow \mathbb{C}^{d}$ a projeçāo natural. Segundo W.L.Marar [21] existem vizinhanças $W$ e $Z$ da origem em $\mathbb{C}^{p}$ e 
$\mathbb{C}^{d}$ respectivamente, tais que $W \times Z \subset V$ e $F \mid F^{-1}(W \times Z): F^{-1}(W \times Z) \rightarrow$ $W \times Z$ é um representante finito de $F$ e a restrição da projeção $\pi: W \times Z \rightarrow Z$ a $I_{\text {rel }}(F) \cap(W \times Z)$ é própria e finita a um. Um tal representante é chamado um bom representante.

Teorema 4.2.2 ([21], Teorema 4.1) Seja $F: F^{-1}(W \times Z) \rightarrow W \times Z$ um bom representante de um desdobramento $\mathcal{A}$-versal $F$ de $f$ como acima. Então existem $\epsilon>0$ e uma vizinhança $T \subset Z \subset \mathbb{C}^{d}$ da origem, tais que a restrição $\left.F: F^{-1}\left(\pi^{-1}(T \backslash \Sigma)\right) \rightarrow Y \cap\left(B_{\epsilon} \times T \backslash \Sigma\right)\right)$ é topologicamente localmente trivial sobre $T \backslash \Sigma$, com respeito a projeção $\pi: B_{\varepsilon} \times(T \backslash \Sigma) \rightarrow T \backslash \Sigma$ onde $B_{\varepsilon}$ é a bola fechada em $\mathbb{C}^{p}$ de centro na origem e raio $\epsilon, \Sigma \subset \mathbb{C}^{d}$ é o conjunto de bifurcação de $F$ e $Y$ é a imagem de $F$.

Corolário 4.2.3 ([21], Corolário 4.2) São consequências imediatas de 4.2.2:

(i) As aplicações $f_{t}: F^{-1}\left(\pi^{-1}(t)\right) \rightarrow Y \cap\left(B_{\epsilon} \times\{t\}\right)$ têm o mesmo tipo topológico, para todo $t \in T \backslash \Sigma$.

(ii) $\pi \mid Y \cap\left(B_{\epsilon} \times(T \backslash \Sigma)\right): Y \cap\left(B_{\epsilon} \times(T \backslash \Sigma)\right) \rightarrow T \backslash \Sigma$ é a projeção de uma fibração localmente trivial. A fibra $Y_{t}=Y \cap\left(B_{\epsilon} \times\{t\}\right)$ sobre $t \in T \backslash \Sigma$ é a imagem da aplicação $f_{t}$ acima.

(iii) $\pi \circ F: F^{-1}\left(\pi^{-1}(T \backslash \Sigma) \rightarrow T \backslash \Sigma\right.$ é a projeção de uma fibração localmente trivial. A fibra $X_{t}=F^{-1}\left(\pi^{-1}(t)\right)$ é o dominio de $f_{t}$.

Definição 4.2.4 A aplicação $f_{t}: X_{t} \rightarrow Y_{t}$ é dita uma perturbação estável de $f$.

Segue de [28] que $Y_{t}$ tem o tipo de homotopia de um bouquet de $n$ esferas. O número de esferas no bouquet é chamado de número de Milnor 
da imagem de $f$ e denotado por $\mu_{I}(f)$. Portanto $\mu_{I}(f)=\operatorname{rank} H_{n}\left(Y_{t} ; \mathbb{Q}\right)$. Para calcular a homologia de $Y_{t}$ usaremos o seguinte método desenvolvido por V.V.Goryunov e D.Mond [16].

Teorema 4.2.5 Seja $f$ como acima. Então $H_{n}\left(Y_{t} ; \mathbb{Q}\right)=\oplus_{p+q=n} E_{p, q}^{\infty}$, onde

$$
E_{p, q}^{1}=A l t_{p+1} H_{q}\left(D^{p+1}\left(f_{t}\right) ; \mathbb{Q}\right)
$$

$e d^{1}=\varepsilon_{*}^{p+1}: E_{p, q}^{1} \rightarrow E_{p-1, q}^{1}$.

\subsection{O número de Milnor da imagem de certos monogermes e trigermes}

Segue de [22] que se $f:\left(\mathbb{C}^{n}, 0\right) \rightarrow\left(\mathbb{C}^{n+1}, 0\right)$ é um monogerme finitamente $\mathcal{A}$-determinado de coposto $1, D^{k}\left(f_{t}\right)$ é uma fibra de Milnor de $D^{k}(f)$, que é uma interseç̧ão completa com singularidade isolada de dimensão $n-k+1$ ou vazio, quando $k \leq n+1$, e consiste no máximo do ponto $\{0\}$ quando $k>n+1$. Então $D^{k}\left(f_{t}\right)$ tem o tipo de homotopia de um bouquet de esferas. O seguinte resultado é o Teorema 2.6 de [16].

Teorema 4.3.1 Nestas circunstâncias, a sequência espectral descrita no Teorema 4.2 .5 colapsa no termo $E^{1}$ e portanto

$$
H_{n}\left(Y_{t} ; \mathbb{Q}\right) \cong \oplus_{k=2}^{n+1} A l t_{k} H_{n-k+1}\left(D^{k}\left(f_{t}\right) ; \mathbb{Q}\right)
$$

Quando $f$ é um multigerme, $D^{k}\left(f_{t}\right)$ é uma união disjunta de fibras de Milnor que correspondem às componentes conexas de $D^{k}(f)$. 
Consideraremos o caso $n=2$. Como monogermes da forma

$$
f(x, y)=\left(x, y^{2}, y p\left(x, y^{2}\right)\right)
$$

e trigermes da forma

$$
g:\left\{\begin{array}{l}
(x, y) \mapsto(x, y, 0) \\
(x, y) \mapsto(x, 0, y) \\
(x, y) \mapsto(x, y, p(x, y))
\end{array}\right.
$$

têm o mesmo conjunto de bifurcação, temos dois fibrados sobre o mesmo espaço base. Além disso em cada fibrado as fibras têm o tipo de homotopia de um bouquet de 2-esferas.

Teorema 4.3.2 $f$ e g têm o mesmo número de Milnor da imagem.

A demonstração do teorema 4.3.2 é baseada nos seguintes lemas.

Definição 4.3.3 $O$ número de Milnor do bordo de $p$ é definido como

$$
\mu_{\delta}(p)=\operatorname{dim} \frac{\mathcal{O}_{2}}{\left(\frac{\partial p}{\partial x}, y \frac{\partial p}{\partial y}\right)}
$$

Observação 4.3.4 O número de Milnor do bordo de $p$ é a $\mathcal{R}^{\delta}$-codimensão de $p$

Lema 4.3.5 Para o monogerme $f(x, y)=\left(x, y^{2}, y p\left(x, y^{2}\right)\right), \mu_{I}(f)=\mu_{\delta}(p)$.

Prova Temos que

$$
H_{1}\left(D^{2}\left(f_{t}\right)\right)=H_{1}^{+}\left(D^{2}\left(f_{t}\right)\right) \oplus H_{1}^{-}\left(D^{2}\left(f_{t}\right)\right)
$$

onde $H_{1}^{+}\left(D^{2}\left(f_{t}\right)\right)=H_{1}\left(D^{2}\left(f_{t}\right) / \mathbb{Z}_{2}\right)$ é a parte invariante com respeito à involução que troca cópias de $\mathbb{C}^{2}$ e $H_{1}^{-}\left(D^{2}\left(f_{t}\right)\right)=A l t_{2} H_{1}\left(D^{2}\left(f_{t}\right)\right)$ é a parte alternada. Também

$$
D^{2}\left(f_{t}\right)=\left\{(x, y, x,-y) / p_{t}\left(x, y^{2}\right)=0\right\} \cong\left\{(x, y) / p_{t}\left(x, y^{2}\right)=0\right\}
$$




$$
D^{2}\left(f_{t}\right) / \mathbb{Z}_{2} \cong\left\{(x, \xi) / p_{t}(x, \xi)=0\right\}
$$

A involução que age em $D^{2}\left(f_{t}\right)$ se reduz a $(x, y) \mapsto(x,-y)$.

Seja $\ell$ o número de pontos fixos desta ação. Portanto

$$
\ell=\#\left\{(x, 0) / p_{t}(x, 0)=0\right\}
$$

Segue do Teorema do ponto fixo de Lefschetz que

$$
\ell=1-\operatorname{rank} H_{1}^{+}\left(D^{2}\left(f_{t}\right)\right)+\operatorname{rank} H_{1}^{-}\left(D^{2}\left(f_{t}\right)\right)
$$

Aplicando o Teorema 4.2 .5 e observando que $D^{3}\left(f_{t}\right)=\emptyset$ temos que

$$
\begin{aligned}
\mu_{I}(f) & =\operatorname{rank} H_{2}\left(Y_{t} ; \mathbb{Q}\right) \\
& =\operatorname{rank} A l t_{2} H_{1}\left(D^{2}\left(f_{t}\right) ; \mathbb{Q}\right) \\
& =\ell-1+\operatorname{rank} H_{1}^{+}\left(D^{2}\left(f_{t}\right)\right)
\end{aligned}
$$

Como $D^{2}\left(f_{t}\right) / \mathbb{Z}_{2}$ é uma fibra de Milnor de $D^{2}(f) / \mathbb{Z}_{2}$ temos

$$
\mu_{I}(f)=\ell-1+\mu(p)
$$

Segue de [2, pag.211] que

$$
\mu_{\delta}(p)=\operatorname{dim} \frac{\mathcal{O}_{2}}{\left(\frac{\partial p}{\partial x}, \frac{\partial p}{\partial y}\right)}+\operatorname{dim} \frac{\mathcal{O}_{2}}{\left(\frac{\partial p}{\partial x}, y\right)}=\mu(p)+\ell-1
$$

Portanto $\mu_{I}(f)=\mu_{\delta}(p)$.

Lema 4.3.6 Seja g o trigerme

$$
g:\left\{\begin{aligned}
(x, y) & \mapsto(x, y, 0) \\
(x, y) & \mapsto(x, 0, y) \\
(x, y) & \mapsto(x, y, p(x, y))
\end{aligned}\right.
$$

então $\mu_{I}(g)=\mu_{\delta}(p)$ 
Prova Temos que

$$
\begin{aligned}
D^{2}\left(g_{t}\right) \cap\left(U_{1} \times U_{2}\right) & =\{(x, 0, x, 0)\} \cong\{(x, 0)\} \\
D^{2}\left(g_{t}\right) \cap\left(U_{2} \times U_{3}\right) & =\left\{(x, y, x, 0) / y=p_{t}(x, 0)\right\} \cong\left\{(x, y) / y=p_{t}(x, 0)\right\} \\
D^{2}\left(g_{t}\right) \cap\left(U_{1} \times U_{3}\right) & =\left\{(x, y, x, y) / p_{t}(x, y)=0\right\} \cong\left\{(x, y) / p_{t}(x, y)=0\right\} \\
D^{3}\left(g_{t}\right) \cap\left(U_{1} \times U_{2} \times U_{3}\right) & =\left\{(x, 0, x, 0, x, 0) / p_{t}(x, 0)=0\right\} \\
& \cong\left\{(x, 0) / p_{t}(x, 0)=0\right\}
\end{aligned}
$$

Como $D^{1}\left(g_{t}\right)$ consiste da união disjunta de 3 conjuntos abertos $\left(U_{1}, U_{2}, U_{3}\right)$, temos que $A l t_{1} H_{0}\left(D^{1}\left(g_{t}\right) ; \mathbb{Q}\right)=H_{0}\left(D^{1}\left(g_{t}\right) ; \mathbb{Q}\right)=\mathbb{Q}^{3}$. Denotemos por $\left[U_{i}\right]$, $i=1,2,3$, as classes destes abertos.

Denotemos por $\alpha_{i j}, i, j=1,2,3$, a curva $D^{2}\left(g_{t}\right) \cap\left(U_{i} \times U_{j}\right)$. Logo $H_{0}\left(D^{2}\left(g_{t}\right) ; \mathbb{Q}\right)$ é gerado pelas classes destas curvas. Denotemos estas classes por $\left[\alpha_{i j}\right]$. Portanto $A l t_{2} H_{0}\left(D^{2}\left(g_{t}\right) ; \mathbb{Q}\right)=<\left[\alpha_{12}\right]-\left[\alpha_{21}\right],\left[\alpha_{13}\right]-\left[\alpha_{31}\right],\left[\alpha_{23}\right]-$ $\left[\alpha_{32}\right]>$.

Seja

$$
\ell=\#\left\{(x, 0) / p_{t}(x, 0)=0\right\}
$$

Então $D^{3}\left(g_{t}\right) \cap\left(U_{i} \times U_{j} \times U_{s}\right), i, j, s=1,2,3$, consiste de $\ell$ pontos. Denotemos estes pontos por $P_{i j s}^{1}, \ldots, P_{i j s}^{\ell}$. Assim $D^{3}\left(g_{t}\right)$ consiste de $6 \ell$ pontos. Portanto $H_{0}\left(D^{3}\left(g_{t}\right) ; \mathbb{Q}\right)$ é gerado pelas classes destes pontos, que denotaremos da mesma maneira para facilitar a notação, e $A l t_{3} H_{0}\left(D^{3}\left(g_{t}\right) ; \mathbb{Q}\right)=<$ $P_{123}^{1}-P_{132}^{1}-P_{213}^{1}+P_{231}^{1}+P_{312}^{1}-P_{321}^{1}, \ldots, P_{123}^{\ell}-P_{132}^{\ell}-P_{213}^{\ell}+P_{231}^{\ell}+P_{312}^{\ell}-P_{321}^{\ell}>$. 
Logo a página $E^{1}$ da sequência espectral em 4.2 .5 é:

$$
\begin{array}{ccc}
E_{2,0}^{1}=\text { Alt }_{3} H_{0}\left(D^{3}\left(g_{t}\right) ; \mathbb{Q}\right) & =\mathbb{Q}^{\ell} & 0 \\
\lambda \downarrow & \downarrow \\
E_{1,0}^{1}=A l t_{2} H_{0}\left(D^{2}\left(g_{t}\right) ; \mathbb{Q}\right)=\mathbb{Q}^{3} & E_{1,1}^{1}=A l t_{2} H_{1}\left(D^{2}\left(g_{t}\right) ; \mathbb{Q}\right) \\
\delta \downarrow & \downarrow \\
E_{0,0}^{1}=A l t_{1} H_{0}\left(D^{1}\left(g_{t}\right) ; \mathbb{Q}\right)=\mathbb{Q}^{3} & 0
\end{array}
$$

Como

$\lambda\left(P_{123}^{k}-P_{132}^{k}-P_{213}^{k}+P_{231}^{k}+P_{312}^{k}-P_{321}^{k}\right)=\left[\alpha_{23}\right]-\left[\alpha_{32}\right]-\left[\alpha_{13}\right]+\left[\alpha_{31}\right]+\left[\alpha_{12}\right]-\left[\alpha_{12}\right]$

para todo $k=1, \ldots, \ell$, temos que

$$
\left\{\begin{array}{l}
\operatorname{dim} \operatorname{Im} \lambda=1 \\
\operatorname{dim} \operatorname{ker} \lambda=\ell-1
\end{array}\right.
$$

Como

$$
\begin{aligned}
& \delta\left(\left[\alpha_{12}\right]-\left[\alpha_{21}\right]\right)=\left[U_{1}\right]-\left[U_{2}\right] \\
& \delta\left(\left[\alpha_{13}\right]-\left[\alpha_{31}\right]\right)=\left[U_{1}\right]-\left[U_{3}\right] \\
& \delta\left(\left[\alpha_{23}\right]-\left[\alpha_{32}\right]\right)=\left[U_{2}\right]-\left[U_{3}\right]
\end{aligned}
$$

segue que

$$
\left\{\begin{array}{l}
\operatorname{dim} \operatorname{Im} \delta=2 \\
\operatorname{dim} \operatorname{ker} \delta=1
\end{array}\right.
$$

Logo a página $E^{2}$ da sequência espectral é:

$$
\begin{array}{cc}
\mathbb{Q}^{\ell-1} & 0 \\
0 & A l t_{2} H_{1}\left(D^{2}\left(g_{t}\right) ; \mathbb{Q}\right) \\
& \\
\mathbb{Q} & 0
\end{array}
$$


Assim a sequência colapsa em $E^{2} \mathrm{e}$

$$
H_{2}\left(Y_{t} ; \mathbb{Q}\right) \cong \mathbb{Q}^{\ell-1} \oplus \operatorname{Alt}_{2} H_{1}\left(D^{2}\left(g_{t}\right) ; \mathbb{Q}\right)
$$

Mas $A l t_{2} H_{1}\left(D^{2}\left(g_{t}\right) ; \mathbb{Q}\right) \cong H_{1}\left(D^{2}\left(g_{t}\right) \cap\left(U_{1} \times U_{3}\right) ; \mathbb{Q}\right)$. Portanto

$$
\mu_{I}(g)=\operatorname{rank} H_{2}\left(Y_{t} ; \mathbb{Q}\right)=\ell-1+\mu(p)=\mu_{\delta}(p)
$$

\subsection{Invariantes}

Seja $f:\left(\mathbb{C}^{2}, S\right) \rightarrow\left(\mathbb{C}^{3}, 0\right)$ um multigerme. Denotaremos também por $\mathcal{O}_{\mathbb{C}^{n}, 0}$ o anel local $\mathcal{O}_{n}$ dos germes de funções analíticas de $\left(\mathbb{C}^{n}, 0\right)$ em $\mathbb{C}$. Assim, $\mathcal{O}_{\mathbb{C}^{2}, S}$ é a soma direta de $r$-cópias de $\mathcal{O}_{\mathbb{C}^{2}, 0}$.

Como cross-cap é um fenômeno que ocorre num ramo isoladamente, temos que o número de cross-caps $C$, presentes na imagem de uma perturbação estável de $f$, é a soma dos $C_{i}$, número de cross-caps presentes na imagem de uma perturbação estável de $f^{(i)}$, para $i=1, \ldots, r$. Segundo Mond [27],

$$
C_{i}=\operatorname{dim}_{\mathbb{C}} \frac{\mathcal{O}_{\mathbb{C}^{2}, 0}}{\mathcal{R}_{f^{(i)}}}
$$

onde $\mathcal{R}_{f^{(i)}}$ é o ideal gerado pelos menores $2 \times 2$ da matriz $d f^{(i)}$.

Também segundo Mond, o número de pontos triplos $T$ na imagem de uma perturbação estável de $f$ é dado por

$$
T=\operatorname{dim}_{\mathbb{C}} \frac{\mathcal{O}_{\mathbb{C}^{3}, 0}}{\mathcal{F}_{2}\left(f_{*}\left(\mathcal{O}_{\mathbb{C}^{2}, s}\right)\right)}
$$

onde $f_{*}\left(\mathcal{O}_{\mathbb{C}^{2}, S}\right)$ é $\mathcal{O}_{\mathbb{C}^{2}, S}$ considerado como um $\mathcal{O}_{\mathbb{C}^{3}, 0^{-}}$-módulo, via composição $\operatorname{com} f$, e $\mathcal{F}_{2}\left(f_{*}\left(\mathcal{O}_{\mathbb{C}^{2}, S}\right)\right)$ é o segundo ideal de Fitting. 
Mais geralmente, define-se o $k$-ésimo ideal de Fitting, $\mathcal{F}_{k}\left(f_{*}\left(\mathcal{O}_{\mathbb{C}^{2}, S}\right)\right)$. Seja

$$
\mathcal{O}_{\mathbb{C}^{3}, 0}^{q} \stackrel{\lambda}{\rightarrow} \mathcal{O}_{\mathbb{C}^{3}, 0}^{q} \stackrel{\alpha}{\longrightarrow} \mathcal{O}_{\mathbb{C}^{2}, S} \rightarrow 0
$$

uma apresentação de $\mathcal{O}_{\mathbb{C}^{2}, S}$ sobre $\mathcal{O}_{\mathbb{C}^{3}, 0}$, ou seja, uma sequêcia exata de $\mathcal{O}_{\mathbb{C}^{3}, 0^{-}}$ módulos, onde o morfismo $\alpha$ leva os elementos da base canônica de $\mathcal{O}_{\mathbb{C}^{3}, 0}^{q}$ nos geradores $g_{1}, \ldots, g_{q}$ de $\mathcal{O}_{\mathbb{C}^{2}, S}$, e as colunas da matriz $\lambda$ são as relações entre os $g_{i}$ (com coeficientes em $\mathcal{O}_{\mathbb{C}^{3}, 0}$ ). Definimos $\mathcal{F}_{k}$ como o ideal em $\mathcal{O}_{\mathbb{C}^{3}, 0}$ gerado pelos menores $(q-k) \times(q-k)$ da matriz $\lambda$. Estes ideais independem da escolha da apresentação.

\subsubsection{Bigermes}

1. $f=\left[S_{0} A_{0}\right](k):\left\{\begin{aligned}(x, y) & \mapsto\left(x, y^{2}, x y\right) \\ (x, y) & \mapsto\left(x^{k}, x, y\right)\end{aligned}\right.$

Temos

$$
\left[d f^{(1)}\right]=\left(\begin{array}{cc}
1 & 0 \\
0 & 2 y \\
y & x
\end{array}\right) \quad e \quad\left[d f^{(2)}\right]=\left(\begin{array}{cc}
k x^{k-1} & 0 \\
1 & 0 \\
0 & 1
\end{array}\right)
$$

Logo $C_{1}=\operatorname{dim}_{\mathbb{C}} \frac{\mathcal{O}_{\mathrm{C}^{2}, 0}}{\langle x, y\rangle}=1$ e $C_{2}=0$.

Como $\mathcal{O}_{\mathbb{C}^{2}} / f_{*}^{(1)}\left(O_{\mathbb{C}^{2}, 0}\right)$ é dado por

$$
\frac{\mathcal{O}_{\mathbb{C}^{2}}}{<x, y^{2}, x y>}
$$

segue do Teorema da Preparação de Malgrange (ver por exemplo [15, Teorema.3.6]) que $\mathcal{O}_{\mathbb{C}^{2}}$ é gerado (como um $\mathcal{O}_{\mathbb{C}^{3}}$-módulo via $f^{(1)}$ ) por 1 e $y$. Via $f^{(2)}, \mathcal{O}_{\mathbb{C}^{2}}$ é gerado por 1 . Logo $\mathcal{O}_{\mathbb{C}^{2}, S}$ é gerado por $(1,0),(y, 0),(0,1)$. As relações são:

$$
\begin{array}{r}
X Y(1,0)-Z(y, 0)=0 \\
Z(1,0)-X(y, 0)=0 \\
\left(X-Y^{k}\right)(0,1)=0
\end{array}
$$


Assim, o segundo ideal de Fitting, $\mathcal{F}_{2}\left(f_{*}\left(\mathcal{O}_{2} \oplus \mathcal{O}_{2}\right)\right)$, é gerado pelos menores $1 \times 1$ da matriz

$$
\left(\begin{array}{ccc}
X Y & -Z & 0 \\
Z & -X & 0 \\
0 & 0 & X-Y^{k}
\end{array}\right)
$$

Portanto $T=\operatorname{dim} \mathcal{O}_{3} / \mathcal{F}_{2}\left(f_{*}\left(\mathcal{O}_{2} \oplus \mathcal{O}_{2}\right)\right)=k$.

2. $f=\left[S_{0} A_{k}\right](1):\left\{\begin{array}{l}(x, y) \mapsto\left(x, y^{2}, x y\right) \\ (x, y) \mapsto\left(x, x^{k+1}, y\right)\end{array}\right.$

Análogo a 1., temos que $C_{1}=1$ e como $f^{(2)}$ é uma imersão, $C_{2}=0$.

Também, $\mathcal{O}_{\mathbb{C}^{2}, S}$ é gerado por $(1,0),(y, 0),(0,1)$. As relações são:

$$
\begin{aligned}
X Y(1,0)-Z(y, 0) & =0 \\
Z(1,0)-X(y, 0) & =0 \\
\left(X^{k+1}-Y\right)(0,1) & =0
\end{aligned}
$$

Assim, o segundo ideal de Fitting, $\mathcal{F}_{2}\left(f_{*}\left(\mathcal{O}_{2} \oplus \mathcal{O}_{2}\right)\right)$, é gerado pelos menores $1 \times 1$ da matriz

$$
\left(\begin{array}{ccc}
X Y & -Z & 0 \\
Z & -X & 0 \\
0 & 0 & X^{k+1}-Y
\end{array}\right)
$$

Portanto $T=\operatorname{dim} \mathcal{O}_{3} / \mathcal{F}_{2}\left(f_{*}\left(\mathcal{O}_{2} \oplus \mathcal{O}_{2}\right)\right)=1$.

3. $f=\left[S_{0} A_{k}\right](k+1):\left\{\begin{array}{l}(x, y) \mapsto\left(x, y^{2}, x y\right) \\ (x, y) \mapsto\left(x, y, y^{k+1}\right)\end{array}\right.$

Analogamente temos que $C=1$ e $T=k+1$.

4. $f=\left[S_{k} A_{0}\right]:\left\{\begin{array}{l}(x, y) \mapsto\left(x, y^{2}, y^{3}+x^{k+1} y\right) \\ (x, y) \mapsto(0, x, y)\end{array}\right.$

Temos $C_{1}=k+1$ e $C_{2}=0$. 
Via $f^{(1)}, \mathcal{O}_{\mathbb{C}^{2}}$ é gerado (como um $\mathcal{O}_{\mathbb{C}^{3}}$-módulo) por 1 e $y$. Via $f^{(2)}, \mathcal{O}_{\mathbb{C}^{2}}$ é gerado por 1 . Logo $\mathcal{O}_{\mathbb{C}^{2}, S}$ é gerado por $(1,0),(y, 0),(0,1)$. As relações são:

$$
\begin{array}{r}
Z(1.0)-\left(Y+X^{k+1}\right)(y, 0)=0 \\
\left(Y^{2}+X^{k+1} Y\right)(1,0)-Z(y, 0)=0 \\
X(0,1)=0
\end{array}
$$

Assim, o segundo ideal de Fitting, $\mathcal{F}_{2}\left(f_{*}\left(\mathcal{O}_{2} \oplus \mathcal{O}_{2}\right)\right)$, é gerado pelos menores $1 \times 1$ da matriz

$$
\left(\begin{array}{ccc}
Z & -Y-X^{k+1} & 0 \\
Y^{2}+X^{k+1} Y & -Z & 0 \\
0 & 0 & X
\end{array}\right)
$$

Portanto $T=\operatorname{dim} \mathcal{O}_{3} / \mathcal{F}_{2}\left(f_{*}\left(\mathcal{O}_{2} \oplus \mathcal{O}_{2}\right)\right)=1$.

5. Para os bigermes da forma

$$
f:\left\{\begin{aligned}
(x, y) & \mapsto(x, y, 0) \\
(x, y) & \mapsto(x, y, \varphi(x, y))
\end{aligned}\right.
$$

é imediato que $C=T=0$.

Concluindo, temos

\begin{tabular}{|c|c|c|c|}
\hline & $\mathcal{A}_{e}-\operatorname{cod}$ & $C$ & $T$ \\
\hline$\left[S_{0} A_{0}\right](k)$ & $k$ & 1 & $k$ \\
\hline$\left[S_{0} A_{k}\right](1)$ & $k+1$ & 1 & 1 \\
\hline$\left[S_{0} A_{k}\right](k-1)$ & $k+2$ & 1 & $k+1$ \\
\hline$\left[S_{k} A_{0}\right]$ & $k+1$ & $k+1$ & 1 \\
\hline
\end{tabular}




\subsubsection{Trigermes}

Consideremos os trigermes da forma

$$
f:\left\{\begin{aligned}
(x, y) & \mapsto(x, y, 0) \\
(x, y) & \mapsto(x, 0, y) \\
(x, y) & \mapsto(x, y, p(x, y))
\end{aligned}\right.
$$

Como cada ramo é uma imersão temos que $C=0$. É imediato que $\mathcal{O}_{\mathbb{C}^{2}, s}$ é

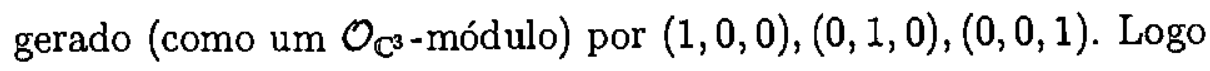

$$
T=\operatorname{dim} \frac{\mathcal{O}_{\mathbb{C}^{3}}}{<Z, Y, Z-p(X, Y)\rangle}=\operatorname{dim} \frac{\mathcal{O}_{\mathbb{C}}}{<p(X, 0)\rangle}
$$

A seguir analisaremos os trigermes simples.

1. $f=\left[S_{k}\right]$. Neste caso $p(x, y)=y+x^{k+1}$. Logo $T=k+1$.

2. $f=\left[B_{k}\right]$. Neste caso $p(x, y)=x^{2}+y^{k}$. Logo $T=2$.

3. $f=\left[C_{k}\right]$. Neste caso $p(x, y)=x y+x^{k}$. Logo $T=k$.

4. $f=\left[F_{4}\right]$. Neste caso $p(x, y)=x^{3}+y^{2}$. Logo $T=3$

Concluindo, temos

\begin{tabular}{|c|c|c|c|}
\hline & $\mathcal{A}_{e}-\operatorname{cod}$ & $C$ & $T$ \\
\hline$\left[S_{k}\right]$ & $k$ & 0 & $k+1$ \\
\hline$\left[B_{k}\right]$ & $k$ & 0 & 2 \\
\hline$\left[C_{k}\right]$ & $k$ & 0 & $k$ \\
\hline$\left[F_{4}\right]$ & 4 & 0 & 3 \\
\hline
\end{tabular}

Observação 4.4.1 Para o caso de monogermes, em [27] Mond define um terceiro invariante. Como vimos, se $f$ é finitamente determinado, $D^{2}(f)$ é 
uma intersecção completa com singularidade isolada. Temos a ação de $\mathbb{Z}_{2}$ em $D^{2}(f)$, que consiste na permutação dos fatores. Este novo invariante é o número de Milnor da singularidade isolada $D^{2}(f) / \mathbb{Z}_{2}, \mu\left(D^{2}(f) / \mathbb{Z}_{2}\right)$. Neste caso temos o seguinte resultado:

$$
\mu_{I}(f)=C+T+\mu\left(D^{2}(f) / \mathbb{Z}_{2}\right)-1
$$

No caso dos multigermes, $D^{2}(f)$ não é conexo, mas uma união de interseç̧ões completas com singularidades isoladas. Logo não fica claro qual é o invariante correspondente a $\mu\left(D^{2}(f) / \mathbb{Z}_{2}\right)$.

Uma possível definição seria

$$
\mu\left(D^{2}(f) / \mathbb{Z}_{2}\right)=\sum_{i=1}^{r} \mu\left(D^{2}\left(f^{(i)}\right) / \mathbb{Z}_{2}\right)+\sum_{i<j} \mu\left(D^{2}(f) \cap\left(U_{i} \times U_{j}\right)\right)
$$

Em vários exemplos, é possível verificar que dado um número natural positivo $s, s \leq r$, tal que $f^{(i)}$ não é imersão para $i=1, \ldots, s$ e $f^{(i)}$ é imersão para $i=s+1, \ldots, r$, então vale a seguinte relação

$$
\mu_{I}(f)=C+T+\mu\left(D^{2}(f) / \mathbb{Z}_{2}\right)-s-(r-1)(r-2) / 2
$$

Acredito que a utilização do método das sequências espectrais de GoryunovMond pode ser usado para a obtenção deste resultado. 


\section{Capítulo 5}

\section{Multigermes de $\mathcal{A}_{e}$-codimensão}

1

\subsection{Introdução}

Em sua tese de doutorado na Universidade de Warwick, Inglaterra, T.Cooper mostrou que os multigermes de $\mathbb{C}^{n}$ em $\mathbb{C}^{n+1}$ de coposto 1 e $\mathcal{A}_{e}$-codimensão 1 têm número de Milnor da imagem igual a 1 e possuem formas reais com boas perturbações reais. Para multigermes de $\mathbb{C}^{n}$ em $\mathbb{C}^{p}, n \geq p$, o número de Milnor do discriminante é maior ou igual à $\mathcal{A}_{e}$-codimensão e a igualdade ocorre quando o germe é quasihomogêneo (ver [13]). Em [25] D.Mond mostra que monogermes de $\mathbb{C}^{n}$ em $\mathbb{C}^{p}, n \geq p$, de coposto 1 e $\mathcal{A}_{e}$-codimensão 1 possuem formas reais com boas perturbações reais. Tais monogermes são quasihomogêneos, logo possuem número de Milnor do discriminante igual a 1.

Neste capítulo, usando as técnicas de T.Cooper, estendemos o resultado obtido por D.Mond em [25] para multigermes, ou seja, mostramos que 
multigermes de $\mathbb{C}^{n}$ em $\mathbb{C}^{p}: n \geq p$, de coposto 1 e $\mathcal{A}_{e}$-codimensão 1 são quasihomogêneos e possuem formas reais com boas perturbações reais. Este resultado, que será descrito na secção 5.4, foi inspirado no trabalho de Cooper.

Em [10], Cooper desenvolveu um método de classificação de multigermes primitivos de $\mathcal{A}_{e}$-codimensão 1 , que é essencial na demonstração dos fatos acima descritos. Logo, nas secções 5.2 e 5.3 descrevemos tal método. Na seç̧ão 5.2 trataremos da aumentação, que significa essencialmente em dado um multigerme de $\mathcal{A}_{e}$-codimensão 1 , obter um novo multigerme também de $\mathcal{A}_{e}$-codimensão 1 . Nesta secção apresentamos algumas provas mais simples que as de [10]. Na secção 5.3 descrevemos a classificação de multigermes de

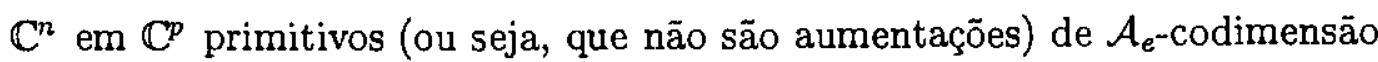
1.

Os resultados que aqui obtivemos, juntamente com os de Cooper, numa nova versão, estão reunidos no artigo [6].

\subsection{Aumentação}

Seja $f:\left(\mathbb{C}^{n}, S\right) \rightarrow\left(\mathbb{C}^{p}, 0\right)$ um multigerme de $\mathcal{A}_{e}$-codimensão 1 , onde $S$ é um subconjunto finito de $\mathbb{C}^{n}$. Seja

$$
\begin{aligned}
F:\left(\mathbb{C} \times \mathbb{C}^{n},\{0\} \times S\right) & \rightarrow\left(\mathbb{C} \times \mathbb{C}^{p},(0,0)\right) \\
(\lambda, x) & \mapsto\left(\lambda, f_{\lambda}(x)\right)
\end{aligned}
$$

um desdobramento $\mathcal{A}_{e}$-miniversal de $f$. Definimos

$$
\begin{aligned}
A_{F} f:\left(\mathbb{C} \times \mathbb{C}^{n},\{0\} \times S\right) & \rightarrow\left(\mathbb{C} \times \mathbb{C}^{p},(0,0)\right) \\
(\lambda, x) & =\left(\lambda, f_{\lambda^{2}}(x)\right)
\end{aligned}
$$


Lema 5.2.1 Se $\alpha:(\mathbb{C}, 0) \rightarrow(\mathbb{C}, 0)$ é bianalítica então existe $\beta:(\mathbb{C}, 0) \rightarrow$ $(\mathbb{C}, 0)$ bianalítica tal que $(\beta(t))^{2}=\alpha\left(t^{2}\right)$.

Prova Seja $\sum_{i=0}^{\infty} a_{i} x^{i}$ a expansão em série de potências de $\alpha$ em torno da origem. Temos que $a_{0}=0$ e $a_{1} \neq 0$. Logo $\gamma=\sum_{i=1}^{\infty} a_{i} x^{i-1}$ define uma função analítica numa vizinhança da origem e $x \cdot \gamma=\alpha$. Como $\gamma(0) \neq 0$, existe um raíz quadrada $\delta$, definida numa vizinhança de $\gamma(0)$, que é uma função analítica. Portanto definimos $\beta(x)=x \cdot\left(\delta \circ \gamma\left(x^{2}\right)\right)$.

Proposição 5.2.2 $A \mathcal{A}$-classe de equivalência de $A_{F} f$ independe da escolha do desdobramento miniversal $F$ de $f$.

Prova Apresentamos aqui uma prova diferente da de [10]. Seja $\tilde{F}=$

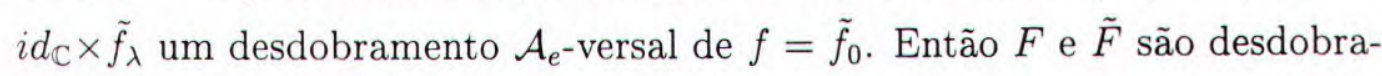
mentos equivalentes, ou seja, existem difeomorfismos

$$
\begin{aligned}
\Phi:\left(\mathbb{C} \times \mathbb{C}^{n},\{0\} \times S\right) & \rightarrow\left(\mathbb{C} \times \mathbb{C}^{n},\{0\} \times S\right) \\
(\lambda, x) & \mapsto\left(\lambda, \phi_{\lambda}(x)\right)
\end{aligned}
$$

$\mathrm{e}$

$$
\begin{aligned}
\Psi:\left(\mathbb{C} \times \mathbb{C}^{p},(0,0)\right) & \rightarrow\left(\mathbb{C} \times \mathbb{C}^{p},(0,0)\right) \\
(\lambda, x) & \mapsto\left(\lambda, \psi_{\lambda}(x)\right)
\end{aligned}
$$

e uma aplicação bianalítica $\alpha:(\mathbb{C}, 0) \rightarrow(\mathbb{C}, 0)$ tais que $\alpha^{*}(F)=\Psi \circ \tilde{F} \circ \Phi$, ou seja,

$$
\left(\lambda, f_{\alpha(\lambda)}(x)\right)=\left(\lambda, \psi_{\lambda}\left(\tilde{f}_{\lambda}\left(\phi_{\lambda}(x)\right)\right)\right)
$$

Definimos

$$
\begin{aligned}
A_{\Phi}:\left(\mathbb{C} \times \mathbb{C}^{n},\{0\} \times S\right) & \rightarrow\left(\mathbb{C} \times \mathbb{C}^{n},\{0\} \times S\right) \\
(\lambda, x) & \mapsto\left(\lambda, \phi_{\lambda^{2}}(x)\right)
\end{aligned}
$$

e

$$
\begin{aligned}
A_{\Psi}:\left(\mathbb{C} \times \mathbb{C}^{p},(0,0)\right) & \rightarrow\left(\mathbb{C} \times \mathbb{C}^{p},(0,0)\right) \\
(\lambda, x) & \mapsto\left(\lambda, \psi_{\lambda^{2}}(x)\right)
\end{aligned}
$$


Segue do Lema 5.2.1 que existe $\beta:(\mathbb{C}, 0) \rightarrow(\mathbb{C}, 0)$, bianalítica, tal que $\beta(\lambda)^{2}=\alpha\left(\lambda^{2}\right)$. Logo temos que

$$
\beta^{*}\left(A_{F} f\right)=\mathcal{A}_{\Psi} \circ A_{\tilde{F}} f \circ A_{\Phi}
$$

Assim, $A_{F} f$ e $A_{\tilde{F}} f$ são desdobramentos equivalentes e portanto são germes $\mathcal{A}$-equivalentes.

Denotaremos por $A f$ a $\mathcal{A}$-classe de equivalência de $A_{F} f$.

Proposição 5.2.3 Se $f$ é A-equivalente a $f^{\prime}$ entâo $A f=A f^{\prime}$.

Prova Se $f$ é $\mathcal{A}$-equivalente a $f^{\prime}$ então existem germes de aplicações bianalíticas $\phi:\left(\mathbb{C}^{n}, S\right) \rightarrow\left(\mathbb{C}^{n}, S\right)$ e $\psi:\left(\mathbb{C}^{p}, 0\right) \rightarrow\left(\mathbb{C}^{p}, 0\right)$ tais que $\psi \circ f=f^{\prime} \circ \phi$. Logo $F^{\prime}=\left(\psi \times i d_{\mathbb{C}}\right) \circ F \circ\left(\phi^{-1} \times i d_{\mathbb{C}}\right)$ é um desdobramento versal de $f^{\prime}$ e $\left(\psi^{-1} \times i d_{\mathbb{C}}\right) \circ A_{F^{\prime}} f^{\prime} \circ\left(\phi \times i d_{\mathbb{C}}\right)=A_{F} f$. Logo $A_{F^{\prime}} f^{\prime}$ é $\mathcal{A}$-equivalente a $A_{F} f$ e portanto $A f=A f^{\prime}$.

Definição 5.2.4 Af é dita aumentaçāo de $f$. Dizemos também que um multigerme é uma aumentação se é uma aumentação de algum multigerme f. Um multigerme que não é uma aumentação é dito primitivo.

Exemplo 5.2.5 Os multigermes de $\mathcal{A}_{e}$-codimensāo 1 de $\mathbb{C}^{2}$ em $\mathbb{C}^{2}$ são:

I. A lips: $(x, y) \mapsto\left(x, y^{3}+x^{2} y\right)$.

II. O rabo de andorinha: $(x, y) \mapsto\left(x, y^{4}+x y\right)$.

III. O bigerme consistindo de duas dobras tangentes: $\left\{\begin{aligned}(x, y) & \mapsto\left(x, y^{2}\right) \\ (x, y) & \mapsto\left(x, x^{2}+y^{2}\right)\end{aligned}\right.$ IV. Um trigerme consistindo de três dobras cujos discriminantes são dois a dois transversais. 
V. O bigerme consistindo de uma dobra e uma cúspide, com o discriminante da dobra transversal à reta tangente limite do discriminante da cúspide.

I é aumentaçāo de $y \mapsto y^{3}$; II é primitivo; III é aumentação do bigerme consistindo de dois ramos $x \mapsto x^{2}$ e $\bar{x} \mapsto \bar{x}^{2}$; IV e V são primitivos.

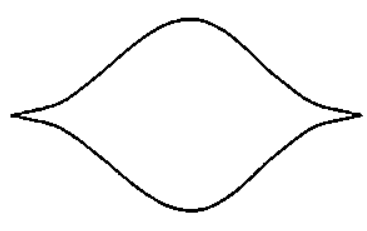

I

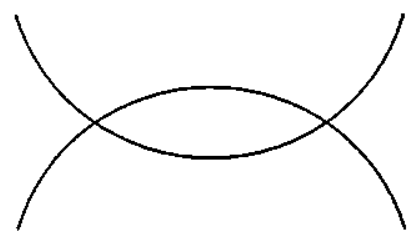

III

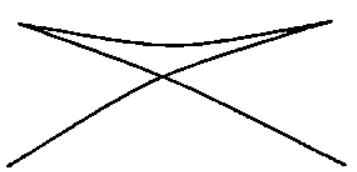

II

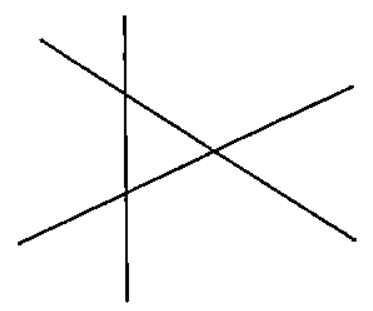

IV

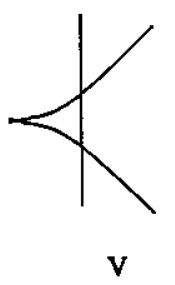

$\mathrm{V}$

Figura 5.1: Discriminantes de boas perturbações reais de germes de codimensão 1 do plano no plano

A prova apresentada por Cooper para o teorema seguinte consiste no cálculo do espaço tangente. Assim, o lema que vem imediatamente depois segue como um corolário. Apresentamos aqui uma prova usando a teoria de $\mathcal{K}_{V}$-equivalência desenvolvida por J.Damon (ver [11]).

Teorema 5.2.6 Af tem $\mathcal{A}_{e}$-codimensão 1 . 
Prova O diagrama

$$
\begin{array}{rrr}
\mathbb{C}^{n+1} & \stackrel{F}{\rightarrow} & \mathbb{C}^{p+1} \\
& & \uparrow \gamma \\
\mathbb{C}^{n+1} & \stackrel{A_{F} f}{\rightarrow} & \mathbb{C}^{p+1}
\end{array}
$$

onde $\gamma(\delta, y)=\left(\delta^{2}, y\right)$, é um pullback. Segue de [11] que a $\mathcal{A}_{e}$-codimensão de

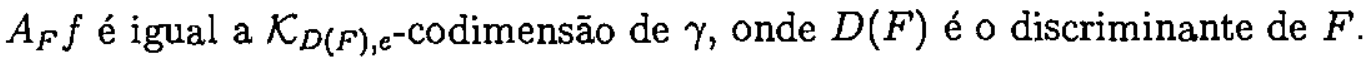

Mas o diagrama

$$
\begin{array}{rrrr}
\mathbb{C}^{n+1} & \stackrel{F}{\rightarrow} & \mathbb{C}^{p+1} \\
& & \uparrow i \\
\mathbb{C}^{n} & \stackrel{f}{\rightarrow} & \mathbb{C}^{p}
\end{array}
$$

onde $i$ é a inclusão, é também um pullback. Assim a $\mathcal{K}_{D(F), e^{-c o d i m e n s a ̃ o ~ d e ~}}$ $i$ é igual a $\mathcal{A}_{e}$-codimensão de $f$ e portanto é 1 .

Como $i$ é a inclusão natural, temos que

$$
N \mathcal{K}_{D(F), e^{i}}=\frac{\theta(i)}{t i(\theta(p))+i^{*}(\operatorname{Der}(\log D(F)))}=\frac{\mathcal{O}_{p}}{d \lambda\left(i^{*}(\operatorname{Der}(\log D(F)))\right)}
$$

(onde $d \lambda\left(i^{*}(\operatorname{Der}(\log D(F)))\right)$ ) é o módulo consistindo dos coeficientes de $\partial / \partial \lambda$ dos elementos de $i^{*}(\operatorname{Der}(\log D(F)))$. Analogamente temos que

$$
N \mathcal{K}_{D(F), \mathrm{e}} \gamma=\frac{\mathcal{O}_{p+1}}{d \lambda\left(\gamma^{*}(\operatorname{Der}(\log D(F)))\right)+(\delta)}
$$

onde o $(\delta)$ no denominador vem de $\partial \gamma / \partial \delta$. É claro que $N \mathcal{K}_{D(F), e} i$ e $N \mathcal{K}_{D(F), e} \gamma$ são isomorfos.

Lema 5.2.7 Seja $f:\left(\mathbb{C}^{n}, S\right) \rightarrow\left(\mathbb{C}^{p}, 0\right)$ um multigerme de $\mathcal{A}_{e^{-} \text {-codimensäo } 1}$ e $F:\left(\mathbb{C} \times \mathbb{C}^{n},\{0\} \times S\right) \rightarrow\left(\mathbb{C} \times \mathbb{C}^{p},(0,0)\right), F(\lambda, x)=(\lambda, \tilde{F}(\lambda, x))$ um desdobramento a 1-parâmetro de $f$. Suponhamos $F$ estável (como germe). Então F é versal. 
Prova Como $F$ é estável temos que $T \mathcal{A}_{e} F=\theta(F)$. Logo

$$
\theta(\tilde{F})=\mathbb{C}\left\{\frac{\partial \tilde{F}}{\partial \lambda}\right\}+\tilde{F}^{*}\left(\mathcal{M}_{p}\right) \cdot \frac{\partial \tilde{F}}{\partial \lambda}+t \tilde{F}_{x}\left(\theta(n+1)_{S^{\prime}}\right)+\pi_{2} \omega F(\theta(p+1))
$$

onde $S^{\prime}=\{0\} \times S$ e $\pi_{2}$ é a projeção de $\theta(F)$ em $\theta(\tilde{F})$. Passando ao quociente por $\mathcal{M}_{\mathbb{C}} \theta(\tilde{F})$ temos

$$
\theta(f)=\mathbb{C}\left\{\frac{\partial \tilde{F}}{\partial \lambda}(0, x)\right\}+f^{*}\left(\mathcal{M}_{p}\right) \cdot \frac{\partial \tilde{F}}{\partial \lambda}(0, x)+T \mathcal{A}_{e} f
$$

Como $f$ tem $\mathcal{A}_{e}$-codimensão 1 , existe $v(x)$ tal que

$$
\mathbb{C}\{v(x)\}+T \mathcal{A}_{e} f=\theta(f)
$$

Segue de 5.1 que

$$
v(x)=(a+\alpha(f)) \frac{\partial \tilde{F}}{\partial \lambda}(0, x)\left(\bmod T \mathcal{A}_{e} f\right)
$$

onde $a \in \mathbb{C}$ e $\alpha(f) \in \bar{F}^{*}\left(\mathcal{M}_{p}\right)$. Como $f$ não é estável temos que $a \neq 0$. Logo segue de 5.2 que

$$
\mathbb{C}\left\{\frac{\partial \tilde{F}}{\partial \lambda}(0, x)\right\}+T \mathcal{A}_{e} f=\theta(f)
$$

Portanto $F$ é versal.

O resultado seguinte é uma recíproca do teorema acima.

Proposição 5.2.8 Seja $G=i d_{\mathbb{C}} \times g_{\lambda}$ um desdobramento estável a 1-parâmetro de um multigerme $g=g_{0}$ e suponhamos que $h=i d_{\mathbb{C}} \times g_{\lambda^{2}}$ tenha $\mathcal{A}_{e^{-c o d i-}}$ mensão 1. Então $g$ tem $\mathcal{A}_{e}$-codimensão 1 e $G$ é um desdobramento versal de g. Portanto h é uma aumentação de $g$.

Prova $O$ fato de $g$ ter $\mathcal{A}_{e}$-codimensão 1 segue da prova do Teorema 5.2.6. Segue do Lema 5.2.7 que $G$ é versal. 
Dado um multigerme estável $f:\left(\mathbb{C}^{n}, S\right) \rightarrow\left(\mathbb{C}^{p}, 0\right)$ seja $P f$ o desdobramento trivial a 1-parâmetro de $f$

$$
\begin{aligned}
& P f:\left(\mathbb{C} \times \mathbb{C}^{n},\{0\} \times S\right) \rightarrow\left(\mathbb{C} \times \mathbb{C}^{p},(0,0)\right) \\
& (\lambda, x) \mapsto(\lambda, f(x))
\end{aligned}
$$

Dizemos que um multigerme é um prisma se é $\mathcal{A}$-equivalente a $P g$ para algum multigerme $g$.

Lema 5.2.9 $T \mathcal{A}_{e} P f=\mathcal{O}_{\mathbb{C} \times \mathbb{C}^{n}} \frac{\partial}{\partial \Lambda} \oplus \mathcal{O}_{\mathbb{C}} T \mathcal{A}_{e} f$.

O multigerme $f$ pode ser obtido de $P f$, a menos de $\mathcal{A}$-equivalência, como o pullback do seguinte diagrama

$$
\begin{array}{r}
\mathbb{C} \times \mathbb{C}^{n},\{0\} \times S \stackrel{p_{f}}{\rightarrow} \mathbb{C} \times \mathbb{C}^{p},(0,0) \\
\uparrow i \\
\mathbb{C}^{p}, 0
\end{array}
$$

Proposição 5.2.10 Seja $F(\lambda, x)=\left(\lambda, f_{\lambda}(x)\right)$ um desdobramento $\mathcal{A}_{e}$-versal de um multigerme $f$ de $\mathcal{A}_{e}$-codimensão 1. Então $G(\mu, \lambda, x)=\left(\mu, \lambda, f_{\lambda^{2}+\mu}(x)\right)$ é um desdobramento $\mathcal{A}_{e}$-versal de $g=A_{F} f$.

Prova Segue do Lema 5.2.7.

Como $G(\mu, \lambda, x)=\left(\mu, \lambda, f_{\lambda^{2}+\mu}(x)\right)$ é um desdobramento de $F(\mu, x)=$ $\left(\mu, f_{\mu}(x)\right)$ e $F$ é estável, temos que $G$ é $\mathcal{A}$-equivalente a $P F$. Portanto se um multigerme é uma aumentação, seu desdobramento miniversal é um prisma. A recíproca também é verdadeira:

Teorema 5.2.11 Seja $g$ um multigerme de $\mathcal{A}_{e}$-codimensão 1 e suponhamos que o desdobramento miniversal $G$ de $g$ seja um prisma. Então $g$ é uma aumentação. 
Prova Existe um único número natural $\ell$ e um multigerme estável $h$, único a menos de $\mathcal{A}$-equivalência, tais que $G=i d_{\mathbb{C}} \times g_{\lambda}$ é $\mathcal{A}$-equivalente a $P^{\ell} h$ e $h$ não é um prisma.

O seguinte diagrama é comutativo

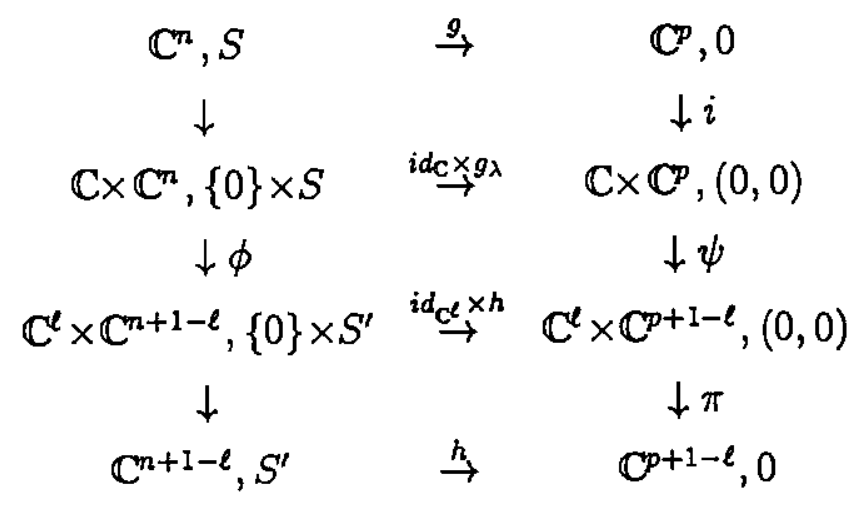

onde $i$ é a inclusão natural, $\phi$ e $\psi$ são bianalíticas, $\pi$ é a projeção natural e $S^{\prime}$ é um subconjunto de $\mathbb{C}^{n+1-\ell}$ de mesma cardinalidade que $S$. Cada um dos 3 retângulos do diagrama é um pullback, assim o retângulo de fora é também

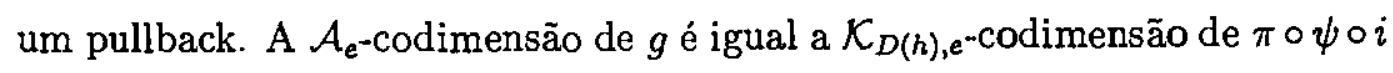
onde $D(h)$ é o discriminante de $h$. Como $h$ é estável, ele é Thom transversal assim qualquer campo vetorial em $\operatorname{Der}(\log D(h))$ levanta, ver 6.14 de [20]. Como $h$ não é um prisma, $\operatorname{Der}(\log D(h)) \subseteq \mathcal{M}_{p+1-\ell} \theta(p+1-\ell)$. Assim,

$$
T \mathcal{K}_{D(h), e}(\pi \circ \psi \circ i) \subseteq T \mathcal{K}_{e}(\pi \circ \psi \circ i)
$$

e a $\mathcal{K}_{e}$-codimensão de $\pi \circ \psi \circ i$ é 0 ou 1 . Não pode ser 1 pois, neste caso, $\pi \circ \psi \circ i$ seria uma submersão e $g$ seria estável. Logo $\pi \circ \psi \circ i$ é $\mathcal{A}$-equivalente a

$$
\left(y_{1}, \ldots, y_{p}\right) \stackrel{\gamma}{\rightarrow}\left(y_{1}, \ldots, y_{p-\ell}, \sum_{i=p+1-\ell}^{p} y_{i}^{2}\right)
$$

Sejam $\Phi$ e $\Psi$ germes de difeomorfismos tais que $\Psi \circ(\pi \circ \psi \circ i)=\gamma \circ \Phi$. 
Seja $\pi_{p+1-\ell}: \mathbb{C}^{p+1-\ell} \rightarrow \mathbb{C}$ a projeção na última coordinada. Então $d\left(\pi_{p+1-\ell} \circ \Psi \circ(\pi \circ \psi \circ i)\right)(0)=0$ e como $h$ é transversal a $\pi \circ \psi \circ i$, $d\left(\pi_{p+1-\ell} \circ \Psi \circ h\right)\left(S^{\prime}\right) \neq 0$. Segue que para $\lambda$ numa vizinhança de 0 ,

$$
\left(\pi_{p+1-\ell} \circ \Psi \circ h\right)^{-1}(\lambda) \cong \mathbb{C}^{n-\ell}
$$

$\mathrm{e}\left(\pi_{p+1-\ell} \circ \Psi\right)^{-1}(\lambda) \cong \mathbb{C}^{p-\ell}$.

Definimos $h_{\lambda}=\left.h\right|_{\left(\pi_{p+1-\ell} \circ \Psi \circ h\right)^{-1}(\lambda)}: \mathbb{C}^{n-\ell} \rightarrow \mathbb{C}^{p-\ell}$. Então $h=i d_{\mathbb{C}} \times h_{\lambda}$ é um desdobramento de $h_{0}$. Como o retângulo de fora do diagrama acima é um pullback, $g$ é $\mathcal{A}$-equivalente a $i d_{\mathbb{C}^{\ell}} \times h_{\sum_{i=1}^{\ell} \lambda_{i}^{2}}$. Segue da Proposiçāo 5.2.8 que $g$ é uma aumentação.

\subsection{Classificação de alguns multigermes de $\mathcal{A}_{e}$-codimensão 1}

Nesta seç̧ão analisaremos multigermes de $\mathbb{C}^{n}$ em $\mathbb{C}^{p}$ de $\mathcal{A}_{e}$-codimensāo 1.0 objetivo é mostrar que a classificação de tais multigermes se reduz a questões acerca de monogermes. Isto é análogo à seç̧ão 1 de [23] onde Mather descreve um multigerme estável em termos de monogermes estáveis. Descrevemos aqui os resultados (e quase todas as provas) do Capítulo 4 de [10].

O papel do ramos submersivos na classificação e teoria do desdobramento dos multigermes é trivial. Por esta razão "um multigerme com $r$ ramos"significa um multigerme com $r$ ramos não submersivos.

Dado um multigerme $f:\left(\mathbb{C}^{n}, S\right) \rightarrow\left(\mathbb{C}^{p}, 0\right)$ com ramos $f^{(1)}, \ldots, f^{(r)}$, definimos

$$
\tau(f)=e v_{0}\left[(\omega f)^{-1}\left\{f^{*} \mathcal{M}_{p} \theta(f)+t f\left(\theta(n)_{S}\right)\right\}\right]
$$


onde $e v_{0}: \theta(p) \rightarrow T_{0} \mathbb{C}^{p}$ é dada por $e v_{0}(\eta)=\left.\eta\right|_{0}$. Definimos também

$$
\tau^{\prime}(f)=e v_{0}\left[(\omega f)^{-1}\left\{t f\left(\theta(n)_{S}\right)\right\}\right]
$$

Então $\tau^{\prime}(f)=e v_{0}(\operatorname{Der}(\log D(f))$ onde $D(f)$ é o discriminante de $f$ (quando $n<p$ discriminante significa imagem).

Um conjunto finito $E_{1}, \ldots, E_{r}$ de subespaços vetoriais de um espaço vetorial $F$ de dimensão finita tem intersecção regular (com relação a $F$ ) se

$$
\operatorname{codim}\left(E_{1} \cap \ldots \cap E_{r}\right)=\operatorname{codim} E_{1}+\cdots+\operatorname{codim} E_{r}
$$

Lema 5.3.1 $E_{1}, \ldots, E_{\mathrm{r}}$ tem intersecção regular se, e somente se, a aplicação natural

$$
F \rightarrow\left(F / E_{1}\right) \oplus \ldots \oplus\left(F / E_{r}\right)
$$

é sobrejetora

O seguinte resultado é devido a Mather [23].

Proposição 5.3.2 O multigerme $f$ é estável se, e somente se, cada ramo $f^{(i)}$ é estável e $\tau\left(f^{(1)}\right), \ldots, \tau\left(f^{(r)}\right)$ tem intersecção regular com relação a $T_{0} \mathbb{C}^{p}$. Além disso, $\tau(f)=\tau\left(f^{(1)}\right) \cap \ldots \cap \tau\left(f^{(r)}\right)$.

A seguir investigaremos o significado geométrico de $\tau^{\prime}$.

Lema 5.3.3 Se $f:\left(\mathbb{C}^{n}, S\right) \rightarrow\left(\mathbb{C}^{p}, 0\right)$ é estável então $\tau(f)=\tau^{\prime}(f)$.

Lema 5.3.4 Se $f=i d_{\mathbb{C}^{m}} \times g$ (ou seja, $f=P^{m} g$ ) então $\tau^{\prime}(f)=T_{0} \mathbb{C}^{m} \oplus \tau^{\prime}(g)$. 
Lema 5.3.5 $S e \operatorname{dim}_{\mathbb{C}} \tau^{\prime}(f)=m$, então existe um germe $g$, que não é um prisma, e difeomorfismos $\phi$ e $\psi$ tais que $f=\psi \circ\left(i d_{\mathbb{C}^{m}} \times g\right) \circ \phi$, ou seja, $f$ é $\mathcal{A}$-equivalente a $P^{m} g$ e $\tau^{\prime}(f)=d \psi_{0}\left(T_{0} \mathbb{C}^{m}\right)$.

Prova Suponhamos que $t f(\xi)=\omega f(\eta)$. Se $\eta(0) \neq 0$ então $\xi(s) \neq 0$ para $s \in S$ e as órbitas de $\xi$ e $\eta$ podem ser incorporadas como retas coordenadas num novo sistema de coordenadas em $\left(\mathbb{C}^{n}, S\right)$ e $\left(\mathbb{C}^{p}, 0\right)$ respectivamente; a prova do lema então se reduz ao Lema de Thom-Levine, e $f$ é $\mathcal{A}$-equivalente a $P g_{1}$ para algum germe $g_{1}$. Aplicamos o mesmo procedimento a $g_{1}$. Após $m$ iterações, obtemos $g$ com $\tau^{\prime}(g)=0$ que, portanto, não é um prisma.

Proposiçāo 5.3.6 Sejam $f:\left(\mathbb{C}^{n}, S\right) \rightarrow\left(\mathbb{C}^{p}, 0\right)$ e $g:\left(\mathbb{C}^{m}, T\right) \rightarrow\left(\mathbb{C}^{q}, 0\right)$ multigermes que não são prismas. Suponhamos que $P^{k} f$ é $\mathcal{A}$-equivalente a $P^{\ell} g$. Então $|S|=|T|, n=m, p=q, k=\ell$ e $f$ é $\mathcal{A}$-equivalente a $g$. Além disso, se a $\mathcal{A}$-equivalência entre $P^{k} f$ e $P^{k} g$ é dada por aplicações bianalíticas como no seguinte diagrama então $\psi\left(\mathbb{C}^{k} \times\{0\}\right)=\mathbb{C}^{k} \times\{0\}$

$$
\begin{array}{ccc}
\mathbb{C}^{k} \times \mathbb{C}^{n},\{0\} \times S \stackrel{i d}{\stackrel{\mathfrak{C}^{k}}{\rightarrow} \times f} & \mathbb{C}^{k} \times \mathbb{C}^{p},(0,0) \\
\downarrow \phi & & \downarrow \psi \\
\mathbb{C}^{k} \times \mathbb{C}^{m},\{0\} \times T \stackrel{i d d_{\mathbb{C}^{k}} \times g}{\rightarrow} & \mathbb{C}^{k} \times \mathbb{C}^{q},(0,0)
\end{array}
$$

Segue da Proposição 5.3.6 que, dado um multigerme $f$, existe uma subvariedade maximal bem definida na meta ao longo da qual $f$ é trivial (ou seja, um prisma) conhecida como o estrato analítico de $f$, e coincide com o germe do conjunto dos pontos $y \in\left(\mathbb{C}^{p}, 0\right)$ tais que $f:\left(\mathbb{C}^{n}, f^{-1}(y) \cap C_{f}\right) \rightarrow\left(\mathbb{C}^{p}, y\right)$ é $\mathcal{A}$-equivalente a $f:\left(\mathbb{C}^{n}, S\right) \rightarrow\left(\mathbb{C}^{p}, 0\right)$ onde $C_{f}$ é o conjunto dos pontos críticos de $f$. Além disso, $\tau^{\prime}(f)$ é o espaço tangente ao estrato analítico de $f$ 
em 0 .

Proposição 5.3.7 Dados $f:\left(\mathbb{C}^{n}, S\right) \rightarrow\left(\mathbb{C}^{p}, 0\right)$ e $g:\left(\mathbb{C}^{n}, T\right) \rightarrow\left(\mathbb{C}^{p}, 0\right)$ multigermes, seja $h:\left(\mathbb{C}^{n}, S \cup T\right) \rightarrow\left(\mathbb{C}^{p}, 0\right)$ o multigerme cujos ramos são os de $f$ e g. Suponhamos que $h$ tenha $\mathcal{A}_{e}$-codimensão 1. Se $\psi$ é um germe de uma familia a 1-parâmetro de aplicações bianalíticas em $\left(\mathbb{C}^{p}, 0\right)$ satisfazendo $\psi_{0}=i d_{\mathbb{O}} e$

$$
e v_{0}\left(\left.\frac{d \psi_{t}}{d t}\right|_{t=0}\right) \notin \tau^{\prime}(f)+\tau^{\prime}(g)
$$

então $H:\left(\mathbb{C} \times \mathbb{C}^{n},\{0\} \times(S \cup T)\right) \rightarrow\left(\mathbb{C} \times \mathbb{C}^{p},(0,0)\right)$ cujos ramos são $F=i d_{\mathbb{C}} \times f$ $e G=i d_{\mathbb{C}} \times\left(\psi_{\lambda} \circ g\right)$, é um desdobramento versal de $h$.

Prova Seja $H(\lambda, x)=\left(\lambda, h_{\lambda}(x)\right)$. Se $v=\left.\frac{d h_{\lambda}}{d \lambda}\right|_{\lambda=0} \in T \mathcal{A}_{e} h$, então $v=$ $t h(\xi)+\omega h(\eta)$ para algum $\xi \in \theta(n)_{S \cup T}$ e $\eta \in \theta(p)$. Segue que $\omega f(\eta)=t f(-\xi)$ e $t g(-\xi)=\omega g\left(\eta-\left.\frac{d \psi_{t}}{d t}\right|_{t=0}\right)$ e portanto, $e v_{0}\left(\left.\frac{d \psi_{t}}{d t}\right|_{t=0}\right) \in \tau^{\prime}(f)+\tau^{\prime}(g)$, que é uma contradição.

Como $\left.\frac{d h_{\lambda}}{d \lambda}\right|_{\lambda=0} \notin T \mathcal{A}_{e} h$ e $h$ tem $\mathcal{A}_{e}$-codimensão $1, H$ é um desdobramento versal de $h$.

Corolário 5.3.8 Se $h:\left(\mathbb{C}^{n}, S\right) \rightarrow\left(\mathbb{C}^{p}, 0\right)$ é um multigerme de $\mathcal{A}_{e}$-codimensão 1 , então para todo subconjunto próprio $S^{\prime}$ de $S$, a restrição de $h$ ao multigerme $\left(\mathbb{C}^{n}, S^{\prime}\right) \rightarrow\left(\mathbb{C}^{p}, 0\right)$ é estável.

Prova Seja $S=S^{\prime} \cup S^{\prime \prime}$ onde $S^{\prime} \cap S^{\prime \prime}=\emptyset$. Sejam $h^{\prime}$ e $h^{\prime \prime}$ os multigermes de $h$ em $S^{\prime}$ e $S^{\prime \prime}$ respectivemente. Suponhamos que $h^{\prime}$ ou $h^{\prime \prime}$ não seja estável, digamos $h^{\prime}$. Então $h^{\prime}$ tem $\mathcal{A}_{e}$-codimensão 1 e portanto não é um prisma. Segue do Lema 5.3.5 que $\tau^{\prime}\left(h^{\prime}\right)=0$. Como $h^{\prime \prime}$ não é uma submersão, podemos escolher $v \in T_{0} \mathbb{C}^{p} \backslash \tau^{\prime}\left(h^{\prime \prime}\right)$. Estendemos $v$ a um campo vetorial em $\mathbb{C}^{p}$ e integramos de forma a obter um germe de uma família a 1 parâmetro $\psi_{t}$ de aplicações bianalíticas em $\left(\mathbb{C}^{p}, 0\right)$ satisfazendo as condições 
da Proposição 5.3.7. Portanto $H$, como descrito na Proposição 5.3.7, é um desdobramento versal de $h$. Mas então $i d_{\mathbb{C}} \times h^{\prime}$ é um desdobramento versal de $h^{\prime}$ e portanto $h^{\prime}$ é estável, uma contradição. Portanto $h^{\prime}$ e $h^{\prime \prime}$ são estáveis.

Um conjunto finito $E_{1}, \ldots, E_{r}$ de subespaços vetoriais de um espaço vetorial $F$ de dimensão finita tem intersecção quase regular (com relação a $F)$ se

$$
\operatorname{codim}\left(E_{1} \cap \ldots \cap E_{r}\right)=\operatorname{codim} E_{1}+\cdots+\operatorname{codim} E_{r}-1
$$

Lema 5.3.9 $E_{1}, \ldots, E_{r}$ tem interseç̧ão quase regular se, e somente se, o conúcleo da aplicação natural

$$
F \rightarrow\left(F / E_{1}\right) \oplus \ldots \oplus\left(F / E_{r}\right)
$$

tem dimensão 1 .

Proposição 5.3.10 Seja $h$ como na Proposição 5.3.7. Então $\tau(f)$ e $\tau(g)$ tem intersecção quase regular com relação a $T_{0} \mathbb{C}^{p}$.

Prova Seja $H$ um desdobramento versal de $h$. Restringindo $H$ obtemos desdobramentos versais $F$ de $f$ e $G$ de $g$. Como $f$ é estável, $F$ é equivalente a um prisma sobre $f$ e portanto

$$
T_{0} \mathbb{C}^{p} / \tau(f) \cong T_{0}\left(\mathbb{C} \times \mathbb{C}^{p}\right) / \tau(F)
$$

Temos o seguinte diagrama comutativo

$$
\begin{array}{cccc}
T_{0} \mathbb{C}^{p} & \rightarrow & \frac{T_{0} \mathscr{O}^{p}}{\tau(J)} \oplus \frac{T_{0} \mathscr{O}^{p}}{\tau(g)} \\
\downarrow & & \downarrow & \downarrow \\
T_{0}\left(\mathbb{C} \times \mathbb{C}^{p}\right) & \rightarrow & \frac{T_{0}\left(\mathbb{C} \times \mathbb{O}^{p}\right)}{\tau(F)} \oplus \frac{T_{0}\left(\mathbb{C} \times \mathbb{O}^{p}\right)}{\tau(G)}
\end{array}
$$


no qual a seta vertical direita é bijetiva e a seta horizontal inferior é sobrejetiva por 5.3.1 e 5.3.2. Assim, o conúcleo da seta horizontal superior tem dimensão 1.

Corolário 5.3.11 Se $h$ é um multigerme de $\mathcal{A}_{e}$-codimensão 1 com ramos $h^{(1)}, \ldots, h^{(r)}, r \geq 2$, então $\tau\left(h^{(1)}\right), \ldots, \tau\left(h^{(r)}\right)$ tem interseç̧ão quase regular com relação a $\mathcal{T}_{0} \mathbb{C}^{p}$.

Corolário 5.3.12 Seja $h$ como na Proposição 5.3.7. Então a codimensão de $\tau(f)+\tau(g)$ em $\mathcal{T}_{0} \mathbb{C}^{p}$ é 1 .

É natural perguntar quando podemos dizer que um multigerme de codimensão 1 é primitivo.

Proposição 5.3.13 Seja $h$ como na Proposição 5.3.7. Então $h$ é uma aumentação $k$ vezes de um germe primitivo, onde $k=\operatorname{dim}_{\mathbb{C}}(\tau(f) \cap \tau(g))$.

Prova Segue do Corolário 5.3.11 que podemos escolher $v \in \mathcal{T}_{0} \mathbb{C}^{p} \backslash(\tau(f)+$ $\tau(g))$. Consideremos o germe de uma família a 1-parâmetro $\psi_{t}$ de aplicações bianalíticas em $\left(\mathbb{C}^{p}, 0\right)$ tal que $e v_{0}\left(\left.\frac{d \psi_{t}}{d t}\right|_{t=0}\right)=v$. Temos então um desdobramento versal $H$ de $h$ como na Proposição 5.3.7. Se $\Lambda$ é a primeira coordenada na meta $\mathbb{C} \times \mathbb{C}^{p}$ de $H$ então $\tau(F)=\mathbb{C} \frac{\partial}{\partial \Lambda} \oplus \tau(f)$ e $\tau(G)=\mathbb{C}\left(\frac{\partial}{\partial \Lambda}+v\right) \oplus \tau(g)$. Como $\tau(H)=\tau(F) \cap \tau(G)$ segue que $\tau(H)=\tau(f) \cap \tau(g)$. Portanto, segue da Proposição 5.3.6 que $H$ é um prisma e do Teorema 5.2.11 que $h$ é uma aumentação.

É útil analisarmos multigermes primitivos de $\mathcal{A}_{e}$-codimensão 1 pois, como veremos adiante, resultados sobre germes primitivos geralmente podem ser 
generalizados para suas aumentações. No caso primitivo, Corolário 5.3.12 e Proposição 5.3.13 mostram que temos uma decomposição

$$
T_{0} \mathbb{C}^{p}=\tau(f) \oplus \tau(g) \oplus \mathbb{C} v
$$

Consideremos $\alpha:\left(\mathbb{C}^{a}, 0\right) \rightarrow\left(\mathbb{C}^{p}, 0\right)$ e $\beta:\left(\mathbb{C}^{b}, 0\right) \rightarrow\left(\mathbb{C}^{p}, 0\right)$ parametrizações dos estratos analíticos de $f$ e $g$ respectivamente, onde $a=\operatorname{dim}_{\mathbb{C}} \tau(f)$ e $b=$ $\operatorname{dim}_{\mathbb{C}} \tau(g)$. Fazendo a seguinte mudança de coordenadas:

$$
\begin{aligned}
\psi:\left(\mathbb{C}^{a} \times \mathbb{C}^{b} \times \mathbb{C}, 0\right) & \rightarrow\left(\mathbb{C}^{p}, 0\right) \\
(x, y, \lambda) & \mapsto \alpha(x)+\beta(y)+\lambda v
\end{aligned}
$$

o estrato analítico de $f$ se torna $\mathbb{C}^{a} \times\{0\} \times\{0\}$, o de $g$ se torna $\{0\} \times \mathbb{C}^{b} \times\{0\}$ e $v$ se torna $(0,0,1) \in \mathbb{C}^{a} \times \mathbb{C}^{b} \times \mathbb{C}$. Suponhamos, para o restante desta secção, que esta mudança de coordenada foi realizada.

Dizemos que um multigerme $f$ é transversal a um subespaço vetorial $V$ de $T_{0} \mathbb{C}^{p}$ se todo ramo de $f$ é transversal a $V$. Nossa análise se resume em considerar dois casos: se $g$ é ou não transversal a $\tau(f)$. Primeiramente consideremos o caso $g$ não transversal a $\tau(f)$.

Lema 5.3.14 Um germe estável de coposto zero é uma singularidade de Morse ou o domínio ou o co-domínio tem dimensão zero.

Proposição 5.3.15 Seja $h$ como na Proposição 5.3.7. Suponhamos $h$ primitivo e $g$ não transversal a $\tau(f)$. Então $g$ tem exatamente um ramo que é um prisma sobre uma singularidade de Morse ou uma imersão.

Prova Se $g$ tem mais de um ramo, entāo segue do Corolário 5.3.8 que o multigerme consistindo de $f$ mais um ramo qualquer $g^{(i)}$ de $g$ é estável. Assim $\tau\left(g^{(i)}\right) \pitchfork \tau(f)$, logo $g^{(i)} \pitchfork \tau(f)$. Como este resultado vale para todo $i$ 
temos que $g \pitchfork r(f)$. Mas isto é uma contradição. Logo $g$ tem exatamente um ramo.

Suponhamos que $\operatorname{Im}\left(d g_{0}\right)$ contenha propriamente $\tau(g)$. Então existe uma deformação a 1-parâmetro $h_{t}$ de $h$ que fixa $\operatorname{Im}\left(d g_{0}\right)$ e tal que $g$ seja transversal a $\tau(f)$ para $t \neq 0$. Como $\tau(g)$ continua não transversal a $\tau(f), h_{t}$ não é estável mesmo para $t \neq 0$ e também não é equivalente a $h=h_{0}$. Isto é impossivel pois $h$ tem $\mathcal{A}_{e}$-codimensão 1 . Logo $\operatorname{Im}\left(d g_{0}\right)=\tau(g)$, e portanto $g$ é um prisma sobre um germe de posto 0. Segue do Lema 5.3.14 que $g$ é um prisma sobre uma função de Morse ou uma imersão.

Suponhamos $f, g, h$ como na proposição acima, e suponhamos $g$ um prisma sobre uma singularidade de Morse. Então a codimensão do seu estrato analítico é 1 e portanto $\tau(f)=\{0\}$. Logo temos uma decomposição da meta como $\mathbb{C}^{p-1} \times \mathbb{C}$ onde $\mathbb{C}^{p-1} \times\{0\}$ é o estrato analítico de $g$. Existe uma vizinhança $U$ de 0 em $\mathbb{C}^{p-1}$ tal que para todo $u \in U$, o pullback de $g$ ao longo da inclusão do subconjunto $\{u\} \times \mathbb{C}$ é uma singularidade de Morse e portanto após uma mudança de coordenadas na fonte podemos reduzir este pullback na forma $\sum_{i=1}^{m} x_{i}^{2}$. De fato, podemos dizer mais que isto. As mudanças de coordenadas na fonte dependem analíticamente de $u$ e portanto temos uma mudança de coordenadas na fonte que reduz $g$ à forma

$$
\begin{aligned}
\mathbb{C}^{p-1} \times \mathbb{C}^{m} & \rightarrow \mathbb{C}^{p-1} \times \mathbb{C} \\
\left(\lambda, x_{1}, \ldots, x_{m}\right) & \mapsto\left(\lambda, \sum_{i=1}^{m} x_{i}^{2}\right)
\end{aligned}
$$

Alternativamente se $g$ é uma imersão então sua imagem é seu estrato analítico e podemos fazer uma mudança de coordenadas na fonte de modo que $g$ seja a inclusão de $\mathbb{C}^{b}$ em $\mathbb{C}^{a} \times \mathbb{C}^{b} \times \mathbb{C}$.

Trataremos agora o caso $g$ transversal a $\tau(f)$. Pelo Teorema da Função Implícita podemos decompor a meta $\mathbb{C}^{n}$ de $g$ em $\mathbb{C}^{n-b-1} \times \mathbb{C}^{b} \times \mathbb{C}$ de modo 
que $g$ preserve as últimas $b+1$ coordenadas. Para $\lambda \in \mathbb{C}^{b} \times \mathbb{C}$, definimos $g_{\lambda}: \mathbb{C}^{n-b-1} \rightarrow \mathbb{C}^{a}$ por $g_{\lambda}=\pi \circ g \circ i_{\lambda}$ onde $i_{\lambda}: \mathbb{C}^{n-b-1} \rightarrow \mathbb{C}^{n-b-1} \times \mathbb{C}^{b} \times \mathbb{C}$ é dada por $i_{\lambda}(x)=(x, \lambda)$ e $\pi: \mathbb{C}^{a} \times \mathbb{C}^{b} \times \mathbb{C} \rightarrow \mathbb{C}^{a}$ é a projeção. Então $g=g_{\lambda} \times i d_{\mathbb{C}^{b}} \times \mathbb{C}$ é um desdobramento de $g_{0}$.

Proposiçāo 5.3.16 Nestas circunstâncias, a aplicação

$$
\begin{aligned}
\bar{g}: \mathbb{C}^{n-b-1} \times \mathbb{C} & \rightarrow \mathbb{C}^{a} \times \mathbb{C} \\
(x, \mu) & \mapsto\left(g_{(0, \mu)}(x), \mu\right)
\end{aligned}
$$

onde $(0, \mu) \in \mathbb{C}^{b} \times \mathbb{C}$, é um desdobramento $\mathcal{A}_{\mathbf{e}}$-versal de $g_{0}$.

Prova É suficiente mostrar que se $G: \mathbb{C}^{n-b-1} \times \mathbb{C}^{d} \rightarrow \mathbb{C}^{a} \times \mathbb{C}^{d}$ é um desdobramento de $g_{0}$ então $G$ pode ser induzido de $\bar{g}$. Note que $g_{\lambda}$ é o pullback de $g$ pela inclusão $\gamma_{\lambda}$ de $\mathbb{C}^{a}$ como $\mathbb{C}^{a} \times\{\lambda\} \hookrightarrow \mathbb{C}^{a} \times \mathbb{C}^{b} \times \mathbb{C}$. Também $\bar{g}$ é o pullback de $g$ pela inclusão $\bar{\gamma}$ de $\mathbb{C}^{a} \times \mathbb{C}$ como $\mathbb{C}^{a} \times\{0\} \times \mathbb{C} \hookrightarrow \mathbb{C}^{a} \times \mathbb{C}^{b} \times \mathbb{C}$. Pela Proposição 1.2 de [29], existe uma aplicação $\Gamma: \mathbb{C}^{a} \times \mathbb{C}^{d} \rightarrow \mathbb{C}^{a} \times \mathbb{C}^{b} \times \mathbb{C}$ tal que $\left.\Gamma\right|_{\mathbb{C}^{a} \times\{0\}}=\gamma_{0}$ e tal que o pullback de $g$ por $\Gamma$ é $G$. Definimos

$$
\begin{aligned}
\bar{\omega}: \mathbb{C}^{p} \times \mathbb{C} & \rightarrow \mathbb{C}^{a} \times \mathbb{C}^{b} \times \mathbb{C} \\
(y, \mu) & \mapsto y+(0,0, \mu)
\end{aligned}
$$

Então $\left.\bar{\omega}\right|_{\mathcal{O} \times\{0\}}=i d_{0}$ e $\left.\bar{\omega}\right|_{\mathbb{C}^{a} \times \mathbb{C}}=\bar{\gamma}$ (estamos considerando $\mathbb{C}^{a}$ como um subconjunto de $\mathbb{C}^{p}$ ). Analogamente definimos $\Omega: \mathbb{C}^{p} \times \mathbb{C}^{d} \rightarrow \mathbb{C}^{a} \times \mathbb{C}^{b} \times \mathbb{C}$ tal que $\left.\Omega\right|_{\mathbb{O}_{\times\{0\}}}=i d_{\mathbb{O}^{\infty}}$ e $\left.\Omega\right|_{\mathbb{C}^{a} \times \mathbb{C}^{d}}=\Gamma$.

Consideremos agora dois desdobramentos de $h$. Definimos $H_{\bar{\omega}}: \mathbb{C}^{n} \times \mathbb{C} \rightarrow$ $\mathbb{C}^{p} \times \mathbb{C}$ cujos ramos são $G_{\bar{\omega}}=g \times i d_{\mathbb{C}}$ e $F_{\bar{\omega}}(x, \mu)=(\bar{\omega}(f(x), \mu), \mu)$, e definimos $H_{\Omega}: \mathbb{C}^{n} \times \mathbb{C}^{d} \rightarrow \mathbb{C}^{p} \times \mathbb{C}^{d}$ com ramos $G_{\Omega}=g \times i d_{\mathbb{C}^{d}}$ e $F_{\Omega}(x, \nu)=(\Omega(f(x), \nu), \nu)$.

Segue da Proposição 5.3.7 que $H_{\bar{\omega}}$ é um desdobramento $\mathcal{A}_{e}$-versal de $h$ e portanto existe $\alpha: \mathbb{C}^{d} \rightarrow \mathbb{C}$ tal que $H_{\Omega}$ é isomorfo a $\alpha^{*} H_{\bar{\omega}}$. Note que $g_{0}$ 
é o pullback de $g$ ao longo do estrato analítico de $f$, o desdobramento $\bar{g}$ de $g_{0}$ é o pullback do desdobramento $G_{\bar{\omega}}$ de $g$ ao longo do estrato analítico do desdobramento $F_{\bar{\omega}}$ de $f$ e analogamente o desdobramento $G$ de $g_{0}$ é o pullback do desdobramento $G_{\Omega}$ de $g$ ao longo do estrato analítico do desdobramento $F_{\Omega}$ de $f$. Entretanto, como estratos analíticos e pullbacks são respeitados por $\mathcal{A}$-equivalências, para qualquer multigerme $\hat{h}$, cujos ramos são $\hat{f}$ e $\hat{g}$, a $\mathcal{A}$-classe de equivalência do pullback de $\hat{g}$ ao longo do estrato analítico de $\hat{f}$ é determinado pela $\mathcal{A}$-classe de equivalência de $\hat{h}$. Portanto, $G$ pode ser induzido de $\bar{g}$ por $\alpha$.

Corolário 5.3.17 Com as mesmas hipóteses da Proposição 5.3.16, go é um multigerme primitivo de $\mathcal{A}_{e}$-codimensão 1 e g é um desdobramento $\mathcal{A}_{e}$-versal de $g_{0}$.

Gostaríamos de encontrar uma forma normal para $g$ analogamente ao caso não-transversal, mas não podemos fazer isto para um $g_{0}$ arbitrário.

Definição 5.3.18 Uma aplicação $f: \mathbb{C}^{n} \rightarrow \mathbb{C}^{p}$ é dita quasihomogênea se existem inteiros positivos $\omega_{1}, \ldots, \omega_{n}$ (pesos) e $d_{1}, \ldots, d_{p}$ (graus) tais que para $\mu \in \mathbb{C}, f\left(\mu^{\omega_{1}} x_{1}, \ldots, \mu^{\omega_{n}} x_{n}\right)=\left(\mu^{d_{1}} f_{1}(x), \ldots, \mu^{d_{p}} f_{p}(x)\right)$. Um germe $f$ é dito quasihomogêneo se existe um representante que é quasihomogêneo como uma aplicação. Um multigerme é quasihomogêneo se seus ramos são quasihomogêneos com os mesmos graus.

Seja $f:\left(\mathbb{C}^{n}, S\right) \rightarrow\left(\mathbb{C}^{p}, 0\right)$ um multigerme quasihomogêneo de $\mathcal{A}_{e}$-codimensão 1. Quando o par $(n, p)$ está no domínio das boas dimensões segundo Mather, existe um desdobramento versal quasihomogêneo $F=i d_{\mathbb{C}} \times f_{\lambda}$ de $f$ onde o grau do parâmetro do desdobramento nāo é zero. De fato, se o grau 
é zero, então $F$ é topologicamente trivial e portanto $f$ é topologicamente estável. Mas isto é uma contradição pois nas boas dimensões, topologicamente estável é equivalente a estável. Sejam $\omega, d_{1}, \ldots, d_{p}$ os graus de $F$ e $\omega, \omega_{1}^{(i)}, \ldots, \omega_{n}^{(i)}$ os pesos do $i$-ésimo ramo de $F$. Para $\mu \in \mathbb{C}$ definimos $\psi_{\mu}: \mathbb{C}^{p} \rightarrow \mathbb{C}^{p}$ por $\psi_{\mu}\left(y_{1}, \ldots, y_{p}\right)=\left(\mu^{d_{1}} y_{1}, \ldots, \mu^{d_{p}} y_{p}\right)$ e $\Psi_{\mu}: \mathbb{C}^{p+1} \rightarrow \mathbb{C}^{p+1}$ por $\Psi_{\mu}(\lambda, y)=\left(\mu^{\omega} \lambda, \psi_{\mu}(y)\right)$. Sejam $\phi_{\mu}^{(i)}$ e $\Phi_{\mu}^{(i)}$ os análogos destas aplicações nas fontes dos $i$-ésimos ramos de $f$ e $F$ respectivamente. Se $\phi_{\mu}$ tem ramos $\phi_{\mu}^{(i)}$ então

$$
f_{\mu^{\omega} \lambda} \circ \phi_{\mu}=\psi_{\mu} \circ f_{\lambda}
$$

Lema 5.3.19 Sejam $\bar{f}$ e $\tilde{f}$ multigermes quasihomogêneos de $\mathbb{C}^{n}$ em $\mathbb{C}^{p}((n, p)$ boas dimensões) de $\mathcal{A}_{e}$-codimensão 1. Suponhamos $f$ e $f^{\prime} \mathcal{A}$-equivalentes. Seja $\bar{F}=i d_{\mathbb{C}^{d}} \times \vec{f}_{\lambda}$ um desdobramento versal de $\bar{f}$ com estrato analítico $\{0\} \times \mathbb{C}^{d-1} \times\{0\}$ e $\tilde{F}=i d_{\mathbb{C}} \times \tilde{f}_{\mu}$ um desdobramento miniversal de $\tilde{f}$. Então existem familias de aplicações bianalíticas $\alpha_{\lambda}$ em $\mathbb{C}^{n}$ e $\beta_{\lambda}$ em $\mathbb{C}^{p}, \lambda \in \mathbb{C}^{d}$, tais que o seguinte diagrama comuta

$$
\begin{array}{ccc}
\mathbb{C} \times \mathbb{C}^{d-1} \times \mathbb{C}^{n} & \stackrel{\vec{F}}{\rightarrow} & \mathbb{C} \times \mathbb{C}^{d-1} \times \mathbb{C}^{p} \\
\downarrow \alpha & & \downarrow \beta \\
\mathbb{C} \times \mathbb{C}^{n} & \stackrel{\bar{F}}{\rightarrow} & \mathbb{C} \times \mathbb{C}^{p}
\end{array}
$$

onde $\alpha(\mu, \nu, x)=\left(\mu, \alpha_{(\mu, \nu)}(x)\right)$ e $\beta(\mu, \nu, y)=\left(\mu, \beta_{(\mu, \nu)}(y)\right)$.

Prova Podemos supor que $\tilde{f}$ e $\tilde{F}$ são quasihomogêneos como aplicações.

Sejam $\phi$ e $\psi$ aplicações bianalíticas tais que $\psi \circ \bar{f}=\tilde{f} \circ \phi$. Então $F^{\prime}=$ $\left(i d_{\mathbb{C}^{d}} \times \psi\right) \circ \bar{F} \circ\left(i d_{\mathbb{C}^{d}} \times \phi\right)^{-1}: \mathbb{C}^{d} \times \mathbb{C}^{n} \rightarrow \mathbb{C}^{d} \times \mathbb{C}^{p}$ é um desdobramento versal de $\tilde{f}$ com estrato analítico $\{0\} \times \mathbb{C}^{d} \times\{0\}$.

Como $\tilde{F}$ é um desdobramento miniversal, existe uma submersão $\gamma: \mathbb{C}^{d} \rightarrow$ $\mathbb{C}$ e famílias de aplicações bianalíticas $\bar{\phi}_{\lambda}$ em $\mathbb{C}^{n}$ e $\bar{\psi}_{\lambda}$ em $\mathbb{C}^{p}, \lambda \in \mathbb{C}^{d}$, tais 
que o seguinte diagrama comuta.

$$
\begin{array}{rrr}
\mathbb{C} \times \mathbb{C}^{d-1} \times \mathbb{C}^{n} & \stackrel{F^{\prime}}{\rightarrow} & \mathbb{C} \times \mathbb{C}^{d-1} \times \mathbb{C}^{p} \\
\downarrow \Gamma \times \bar{\phi}_{\lambda} & & \downarrow \Gamma \times \vec{\psi}_{\lambda} \\
\mathbb{C} \times \mathbb{C}^{d-1} \times \mathbb{C}^{n} & \stackrel{\bar{F} \times i d_{\mathbb{C}^{d-1}}}{\rightarrow} & \mathbb{C} \times \mathbb{C}^{d-1} \times \mathbb{C}^{p}
\end{array}
$$

ond $\Gamma(\mu, \nu)=(\gamma(\mu, \nu), \nu)$.

Temos que $\gamma^{-1}(0)=\{0\} \times \mathbb{C}^{d-1}$. Então $\Gamma$ é bianalítica pelo Teorema da Função Inversa. Como $\Gamma$ comuta com a projeção sobre $\mathbb{C}^{d-1}, \Gamma^{-1}$ também comuta, assim existem $\gamma^{\prime}, \gamma^{\prime \prime}: \mathbb{C}^{d} \rightarrow \mathbb{C}$ tais que $\Gamma^{-1}(\mu, \nu)=\left(\gamma^{\prime}(\mu, \nu), \nu\right)$ e $\gamma^{\prime}=\mu \gamma^{\prime \prime}$ onde $\mu: \mathbb{C} \times \mathbb{C}^{d-1} \rightarrow \mathbb{C}$ é a projeção na primeira coordenada. Também $\gamma^{\prime}$ é uma submersão e portanto $\gamma^{\prime \prime}$ é não nula numa vizinhança da origem. Temos que

$$
\left(\gamma^{\prime} \times \psi_{\sqrt[W]{\gamma^{\prime \prime}}}\right) \circ\left(\tilde{F} \times i d_{\mathbb{C}^{d-1}}\right)=\tilde{F} \circ\left(\gamma^{\prime} \times \phi_{\sqrt[W]{\gamma^{n}}}\right)
$$

onde $\phi_{\sqrt[W]{\gamma^{n}}}$ e $\psi_{\sqrt[W]{\gamma^{n}}}$ são como dito antes desta proposição. Então o seguinte diagrama comuta

$$
\begin{array}{ccc}
\mathbb{C} \times \mathbb{C}^{d-1} \times \mathbb{C}^{n} & \stackrel{F^{\prime}}{\rightarrow} & \mathbb{C} \times \mathbb{C}^{d-1} \times \mathbb{C}^{p} \\
\downarrow \bar{\alpha} & & \downarrow \tilde{\beta} \\
\mathbb{C} \times \mathbb{C}^{n} & \stackrel{\bar{F}}{\rightarrow} & \mathbb{C} \times \mathbb{C}^{p}
\end{array}
$$

onde $\bar{\alpha}=\left(\gamma^{\prime} \times \phi \sqrt[w]{\gamma^{n}}\right) \circ\left(\Gamma \times \bar{\phi}_{\lambda}\right)$ e $\bar{\beta}=\left(\left(\gamma^{\prime} \times \psi_{\sqrt[W]{\gamma^{n}}}\right) \circ\left(\Gamma \times \bar{\psi}_{\lambda}\right)\right.$.

A proposição segue tomando $\alpha=\bar{\alpha} \circ(i d \times \phi)$ e $\beta=\bar{\beta} \circ(i d \times \psi)$.

Teorema 5.3.20 Sejam $f:\left(\mathbb{C}^{n}, S\right) \rightarrow\left(\mathbb{C}^{p}, 0\right)$ e $g:\left(\mathbb{C}^{n}, T\right) \rightarrow\left(\mathbb{C}^{p}, 0\right)$ multigermes $\left((n, p)\right.$ boas dimensões). Consideremos $h:\left(\mathbb{C}^{n}, S \cup T\right) \rightarrow\left(\mathbb{C}^{p}, 0\right)$ o multigerme primitivo de $\mathcal{A}_{e}$-codimensão 1 cujos ramos são os de $f$ e g. Suponhamos que 
(a) $g$ não é transversal a $\tau(f)$ ou, $g$ é transversal a $\tau(f)$ e o pullback de $g$ pelo estrato analítico de $f$ é quasihomogêneo.

(b) $f$ não é transversal a $\tau(g)$ ou, $f$ é transversal a $\tau(g)$ e o pullback de $f$ pelo estrato analítico de $g$ é quasihomogêneo.

Então, após mudanças de coordenadas, $f:\left(\mathbb{C}^{n}, S\right) \rightarrow\left(\mathbb{C}^{a} \times \mathbb{C}^{b} \times \mathbb{C}, 0\right) e ́$ um multigerme estável com estrato analítico $\mathbb{C}^{a}, g:\left(\mathbb{C}^{n}, T\right) \rightarrow\left(\mathbb{C}^{a} \times \mathbb{C}^{b} \times \mathbb{C}, 0\right)$ é um multigerme estável com estrato analítico $\mathbb{C}^{b}$ e uma das seguintes afirmações valem para $f$ (também para $g$ trocando $f$ e $g, a$ e b e assim por diante)

(1) $f$ tem somente um ramo:

$$
\begin{aligned}
f:\left(\mathbb{C}^{a}, 0\right) & \rightarrow\left(\mathbb{C}^{a} \times \mathbb{C}^{b} \times \mathbb{C}, 0\right) \\
x & \mapsto(x, 0,0)
\end{aligned}
$$

(2) $b=0$ ef tem somente um ramo:

$$
\begin{aligned}
& f:\left(\mathbb{C}^{a} \times \mathbb{C}^{n-a}, 0\right) \rightarrow\left(\mathbb{C}^{a} \times \mathbb{C}, 0\right) \\
& \left(\lambda, x_{1}, \ldots, x_{n-a}\right) \mapsto\left(\lambda, \sum_{i=1}^{n-a} x_{i}^{2}\right)
\end{aligned}
$$

(3) existe um multigerme primitivo quasihomogêneo $\tilde{f}$ (que pode ser escolhido na $\mathcal{A}$-classe de equivalência do pullback de $f$ pelo estrato analítico de g) e um desdobramento versal $\tilde{F}=i d_{\mathbb{C}} \times \tilde{f}_{\mu}$ de $\tilde{f}$ (que pode ser qualquer desdobramento miniversal de $\tilde{f}$ ) tal que

$$
\begin{aligned}
f:\left(\mathbb{C}^{a} \times \mathbb{C}^{n-a-1} \times \mathbb{C},\{0\} \times S \times\{0\}\right) & \rightarrow\left(\mathbb{C}^{a} \times \mathbb{C}^{b} \times \mathbb{C}, 0\right) \\
(\lambda, x, \mu) & \mapsto\left(\lambda, \tilde{f}_{\mu}(x), \mu\right)
\end{aligned}
$$

Prova $\quad \mathrm{O}$ caso $g$ não transversal a $\tau(f)$ já foi estudado após a Proposição 5.3.15. Note que neste caso a mudança de coordenadas não afeta $f$. 
Suponhamos $g$ transversal a $\tau(f)$ e seja $\tilde{G}=i d \times \tilde{g}_{\mu}$ um desdobramento miniversal de uma forma normal $\tilde{g}$ do pullback de $g$ pelo estrato analítico de $f$. Então após uma mudança de coordenadas paralela ao estrato analítico de $f$, ver Lema 5.3.19, temos

$$
\begin{aligned}
g: \mathbb{C}^{n-b-1} \times \mathbb{C}^{b} \times \mathbb{C} & \rightarrow \mathbb{C}^{a} \times \mathbb{C}^{b} \times \mathbb{C} \\
(z, \delta, \mu) & \mapsto\left(\tilde{g}_{\mu}(z), \delta, \mu\right)
\end{aligned}
$$

Note que os estratos analíticos de $f$ e $g$ não foram mudados.

Normalizamos agora $f$ da mesma maneira. Temos somente que considerar o caso $f$ transversal a $\tau(g)$, pois neste caso $g$ é alterada. Entretanto a alteração decorre de uma mudança de coordenadas na meta paralela ao estrato analítico de $g$ e portanto pode ser desfeita por uma mudança de coordenadas na fonte de $g$.

Finalmente, segue do Lema 5.3.17 que $\tilde{f}$ e $\tilde{g}$ são primitivos.

Observação 5.3.21 Note que podemos ter $g$ não transversal a $\tau(f)$ e $f$ transversal a $\tau(g)$. De fato, seja $h$ o bigerme de $\mathcal{A}_{e}$-codimensão 1 consistindo do cross-cap, $f$, e plano imerso, $g$, se interceptando transversalmente. Sabemos que $h$ é primitivo. Temos que $g$ não é transversal a $\tau(f)=\{0\}$. No entanto $f$ é transversal a $\tau(g)$, que é a imagem de $g$.

O Teorema 5.3.20 mostra que um multigerme de $\mathcal{A}_{e}$-codimensão 1 , satisfazendo certas condições, pode ser reduzido a uma forma normal. O resultado seguinte é a recíproca.

Teorema 5.3.22 Sejam $f$ e $g$ multigermes satisfazendo uma das afirmações (1), (2) e (3) do Teorema 5.3.20. Se h é o multigerme cujos ramos são $f$ e $g$, então $h$ é primitivo quasihomogêneo de $\mathcal{A}_{e}$-codimensão 1. 
Prova Sejam $\lambda_{1}, \ldots, \lambda_{a}, y_{1}, \ldots, y_{b}, \mu$ as coordenadas de $\mathbb{C}^{p}=\mathbb{C}^{a} \times \mathbb{C}^{b} \times \mathbb{C}$. Afrmamos que $\theta(h)=T \mathcal{A}_{e} h+\mathbb{C} \nu$, onde $\nu$ é o campo vetorial ao longo de $h$ que é $\omega f\left(\frac{\partial}{\partial \mu}\right)$ ao longo de $f$ e 0 ao longo de $g$.

De fato, como $\theta(h)=\theta(f) \oplus \theta(g)$ e $f$ e $g$ são estáveis, temos de mostrar que $\omega f(\theta(p)) \subset T \mathcal{A}_{e} h+\mathbb{C} \nu$. É suficiente mostrar que $\omega f\left(\mathcal{M}_{p} \frac{\partial}{\partial \mu}\right) \subset T \mathcal{A}_{e} h$.

Um elemento arbitrario $c$ de $\mathcal{M}_{p}$ é dado por

$$
c=\sum_{i=1}^{a} \alpha_{i} \lambda_{i}+\sum_{j=1}^{b} \beta_{j} y_{j}+m \mu
$$

onde $\alpha_{i}, \beta_{j}, m \in \mathcal{O}_{p}$. Portanto, temos de mostrar que $\omega f\left(\alpha_{i} \lambda_{i} \frac{\partial}{\partial \mu}\right), \omega f\left(\beta_{j} y_{j} \frac{\partial}{\partial \mu}\right)$ e $\omega f\left(m \mu \frac{\partial}{\partial \mu}\right)$ pertencem a $T \mathcal{A}_{e} h$. Mostraremos que o primeiro e o segundo se reduzem ao último caso.

Trataremos primeiro de $\omega f\left(\beta_{j} y_{j} \frac{\partial}{\partial \mu}\right)$. Se $f$ é do tipo (1), este termo é zero. Quando $f$ é do tipo (2), este termo não existe. Resta $f$ do tipo (3). A aplicação $\tilde{f}: \mathbb{C}^{n-a-1} \rightarrow \mathbb{C}^{b}$ é induzida da aplicação estável $\tilde{F}: \mathbb{C}^{n-a-1} \times \mathbb{C} \rightarrow$ $\mathbb{C}^{b} \times \mathbb{C}$ pela inclusão $\gamma: \mathbb{C}^{b} \rightarrow \mathbb{C}^{b} \times \mathbb{C}$. Portanto a $\mathcal{K}_{D(\bar{F})^{-c o d i m e n s a ̃ o ~ d e ~} \gamma \text { é }}$ igual a $\mathcal{A}_{e}$-codimensão de $\tilde{f}$ que é 1 . Ou seja,

$$
\operatorname{dim}_{\mathbb{C}} \frac{\theta(\gamma)}{t \gamma(\theta(b))+\gamma^{*} \operatorname{Der}(\log D(\tilde{F}))}=1
$$

Mas $\gamma^{*} \operatorname{Der}(\log D(\tilde{F})) \subseteq \mathcal{M}_{b} \frac{\partial}{\partial \mu}+t \gamma(\theta(b))$ (caso contrário existiria um elemento em $e v_{0}\left(\operatorname{Der}(\log D(\tilde{F}))=\tau^{\prime}(\tilde{F})\right.$ transversal à imagem de $\gamma$, e portanto $\tilde{F}$ seria um prisma sobre $\tilde{f}$, uma contradição). Logo,

$$
\gamma^{*} \operatorname{Der}(\log D(\tilde{F}))+t \gamma(\theta(b))=\mathcal{M}_{b} \frac{\partial}{\partial \mu}+t \gamma(\theta(b))
$$

Em particular, $y_{j} \frac{\partial}{\partial \mu} \in \gamma^{*} \operatorname{Der}(\log D(\bar{F}))+t \gamma(\theta(b))$ como um campo vetorial ao longo de $\gamma$, digamos $y_{j} \frac{\partial}{\partial \mu}=\eta+\eta^{\prime}$ onde $\eta \in \gamma^{*} \operatorname{Der}(\log D(\tilde{F}))$ e $\eta^{\prime} \in t \gamma(\theta(b))$. 
Retornamos agora a campos vetoriais ao longo de $h$. Em $\mathbb{C}^{a} \times \mathbb{C}^{n-a-1} \times\{0\}$, $\omega f\left(\beta_{j} y_{j} \frac{\partial}{\partial \mu}\right)=\omega f\left(\beta_{j} \eta\right)+\omega f\left(\beta_{j} \eta^{\prime}\right)$. Como $\beta_{j} \eta$ é levantado ao longo de $f, \omega f\left(\beta_{j} \eta\right) \in T \mathcal{A}_{e} h$, e como $\beta_{j} \eta^{\prime}$ não tem componentes na direção $\frac{\partial}{\partial \mu}$, $\omega f\left(\beta_{j} \eta^{\prime}\right) \in T \mathcal{A}_{e} h$. Portanto, para mostrar que $\omega f\left(\beta_{j} y_{j} \frac{\partial}{\partial \mu}\right) \in T \mathcal{A}_{e} h$ é suficiente mostrar que $\omega f\left(m \mu \frac{\partial}{\partial \mu}\right) \in T \mathcal{A}_{e} h$ para algum $m \in \mathcal{O}_{p}$.

Para mostrar o segundo caso, ou seja, $\omega f\left(\alpha_{i} \lambda_{i} \frac{\partial}{\partial \mu}\right) \in T \mathcal{A}_{e} h$, é suficiente mostrar que $\omega g\left(-\alpha_{i} \lambda_{i} \frac{\partial}{\partial \mu}\right) \in T \mathcal{A}_{e} h$, mas isto é análogo ao primeiro caso.

Finalmente, consideremos $\omega f\left(m \mu \frac{\partial}{\partial \mu}\right)$ e $f$ do tipo (3). Podemos considerar $\tilde{f}$ e $\bar{F}$ quasihomogêneo. Como antes temos que $\tilde{F} \circ \Phi_{s}=\Psi_{s} \circ \tilde{F}$, para todo $s \in \mathbb{C}$. Diferenciando com respeito a $s$ temos que $t \tilde{F}(\xi)=\omega \tilde{F}(\eta)$ onde $\xi=\left.\frac{\partial \Phi_{s}}{\partial s}\right|_{s=1}$ e $\eta=\left.\frac{\partial \Psi_{s}}{\partial s}\right|_{s=1}$. Então $\omega f\left(m \mu \frac{\partial}{\partial \mu}\right)-t f\left(\frac{m \circ f}{\omega} \xi\right)$ não tem componente na direção $\frac{\partial}{\partial \mu}$ e portanto está em $T \mathcal{A}_{e} h$. Portanto $\omega f\left(m \mu \frac{\partial}{\partial \mu}\right) \in T \mathcal{A}_{e} h$.

Observação 5.3.23 Se substituírmos $\mathbb{C}$ por $\mathbb{R}$ e aplicações analíticas por analíticas reais, os resultados obtidos até aqui ainda valem a menos das seguintes alterações:

(a) No caso real definimos duas aumentações:

$$
\begin{gathered}
A_{F}^{+}(\lambda, x)=\left(\lambda, f_{\lambda^{2}}(x)\right) \\
A_{F}^{-}(\lambda, x)=\left(\lambda, f_{-\lambda^{2}}(x)\right)
\end{gathered}
$$

(b) Na prova da Proposição 5.3.19, para $\omega$ impar, w/ não está bem definido. Consequentemente temos que definir $\alpha(\mu, \nu, x)=\left(-\mu, \alpha_{(\mu, \nu)}(x)\right) \mathrm{e}$ $\beta(\mu, \nu, y)=\left(-\mu, \beta_{(\mu, \nu)}(y)\right)$ de modo que o diagrama comute.

(c) Teorema 5.3.20 é o mesmo exceto no caso (2) onde $f$ é da forma

$$
f\left(\lambda, x_{1}, \ldots, x_{n-a}\right)=\left(\lambda, \sum_{i=1}^{n-a} \pm x_{i}^{2}\right)
$$


e no caso (3) podemos considerar $f$ da forma $f(\lambda, x, \mu)=\left(\lambda, \tilde{f}_{-\mu}(x), \mu\right)$.

\subsection{Topologia}

Seja $f:\left(\mathbb{C}^{n}, S\right) \rightarrow\left(\mathbb{C}^{p}, 0\right)$ (S um conjunto finito) um multigerme finitamente $\mathcal{A}$-determinado. No parágrafo 4.2 definimos perturbação estável de $f$ no caso $p=n+1$. Analogamente, segundo [13], podemos definir perturbação estável no caso $n \geq p$ trocando imagem por discriminante. Assim, se $F: U \rightarrow$ $V$ é um bom representante de um desdobramento $F:\left(\mathbb{C}^{d} \times \mathbb{C}^{n},\{0\} \times S\right) \rightarrow$ $\left(\mathbb{C}^{d} \times \mathbb{C}^{p},(0,0)\right), F(\lambda, x)=\left(\lambda, f_{\lambda}(x)\right)$, de $f$, o discriminante $D(F)$ de $F$ é uma subvariedade analítica de $V$. Nesta secção consideraremos o caso $n \geq p$, $(n, p)$ boas dimensões.

Consideremos a estratificação canônica de $D(F)$ e seja $\epsilon>0$ tal que para todo $\epsilon^{\prime}$ com $0<\epsilon^{\prime} \leq \epsilon$, todo estrato de $D(f) \cong D(F) \cap\left(\{0\} \times \mathbb{C}^{p}\right)$ é transversal a esfera $S_{\epsilon^{\prime}} \subset \mathbb{C}^{p}$ de centro 0 e raio $\epsilon^{\prime}$ ("stratified transverse"). Tal $\epsilon$ é chamado de raio de Milnor para $D(f)$. Pelo Primeiro Lema de Isotopia de Thom, $D(f) \cap B_{\epsilon}$ é um cone sobre o bordo $D(f) \cap S_{\boldsymbol{\epsilon}}$. Segue que existe $\delta>0$ tal que para $\lambda \in B_{\delta} \subseteq \mathbb{C}, D(F)$ é stratified transverse a $\{\lambda\} \times S_{\epsilon}$ ( $\delta$ é chamado de limite de perturbação para $F$ com respeito a $B_{\epsilon}$ ). Para $\lambda \in B_{\delta}$, o discriminante de $f_{\lambda}$ é definido por $D\left(f_{\lambda}\right) \cap B_{\epsilon}$, ou, em outras palavras, $D(F) \cap\left(\{\lambda\} \times B_{\epsilon}\right)$. Segue de [13] que o discriminante de $f_{\lambda}$ tem o tipo de homotopia de um bouquet de esferas de dimensão $p-1$. O número de esferas do bouquet é chamado número de Milnor do discriminante de $f$ e denotado por $\mu_{\Delta}(f)$.

Dados $\epsilon_{1}, \ldots, \epsilon_{p}>0$ seja $P_{\epsilon_{1}, \ldots, \epsilon_{p}}(0) \circ$ cubo

$$
\left\{\left(y_{1}, \ldots, y_{p}\right) \in \mathbb{C}^{p} /\left|y_{i}\right|<\epsilon_{i} \forall i\right\}
$$


Um pseudo raio de Milnor para $D(f)$ é um $\epsilon>0$ tal que para todo $\epsilon_{1}, \ldots, \epsilon_{p}$ com $0<\epsilon_{i}<\epsilon(\forall i), D(f)$ é "stratified transverse" ao bordo do cubo $P_{\epsilon_{1}, \ldots, \epsilon_{p}}(0)$. Os resultados descritos acima ainda valem se substituírmos $B_{\epsilon}$ por um cubo e o discriminante definido desda maneira é o mesmo.

Seja $\pi: D(F) \rightarrow \mathbb{C}$ a projeção no espaço dos parâmetros $\mathbb{C}$. Entāo $\pi$ induz uma fibração localmente trivial

$$
\left(\left(B_{\delta} \backslash\{0\}\right) \times B_{\epsilon}\right) \cap D(F) \rightarrow B_{\delta} \backslash\{0\}
$$

O seguinte resultado é o Lema 8.1 de [10].

Lema 5.4.1 Sejam $A, A^{\prime}, B, B^{\prime}$ subespaços abertos contráteis de um espaço topológico tais que $A \cap B$ e $A^{\prime} \cap B^{\prime}$ são homotopicamente equivalentes e $A \cap B$ tem vizinhança tubular em $A$ e $B$, e $A^{\prime} \cap B^{\prime}$ tem vizinhança tubular em $A^{\prime}$ e $B^{\prime}$. Então $A \cup B$ e $A^{\prime} \cup B^{\prime}$ são homotopicamente equivalentes.

Suponhamos que $f$ tenha $\mathcal{A}_{e}$-codimensão 1. Seja $F(\lambda, x)=\left(\lambda, f_{\lambda}(x)\right)$ um representante próprio de um desdobramento miniversal de $f$. Para $\mu \in \mathbb{C}$ definimos $g_{\mu}(\lambda, x)=\left(\lambda, f_{\lambda^{2}+\mu}(x)\right)$. Então $G(\mu, \lambda, x)=\left(\mu, \lambda, f_{\lambda^{2}+\mu}(x)\right)$ é um representante próprio de um desdobramento miniversal de $g=A_{F} f$.

Teorema 5.4.2 Com a notação acima o discriminante de $g_{\mu}$ é homotopicamente equivalente à suspensão do discriminante de $f_{\lambda}$.

Prova Seja $\epsilon>0$ um pseudo raio de Milnor para $f$ e $F$ e $\delta>0$ um limite de perturbação para $F$ com respeito a $P_{\epsilon, \ldots, \epsilon}(0) \subseteq \mathbb{C}^{p}$.

Seja $\epsilon^{\prime}>0$ em pseudo raio de Milnor para $g$ e $\delta^{\prime}>0$ um limite de perturbação para $G$ com respeito a $P_{\epsilon^{\prime \prime}, \epsilon, \ldots, \epsilon}(0) \subseteq \mathbb{C}^{p+1}$, onde $\epsilon^{\prime \prime}=\min \{\epsilon, \sqrt{\delta / 2}\}$. 
Seja $\mu_{0} \in \mathbb{C}$ satisfazendo $\mu_{0} \neq 0,\left|\mu_{0}\right|<\delta^{\prime},\left|\mu_{0}\right|<\delta / 2$ e $\left|\mu_{0}\right|<\epsilon^{\prime 2}$. Então $D\left(g_{\mu_{0}}\right)=D\left(g_{\mu_{0}}\right) \cap P_{\epsilon^{\prime \prime}, \epsilon, \ldots, \epsilon}(0)$ é o discriminante de $g_{\mu_{0}}$. Consideremos

$$
\pi: D\left(g_{\mu_{0}}\right) \rightarrow \mathbb{C}
$$

a projeção na primeira coordenada. Logo $\pi\left(D\left(g_{\mu_{0}}\right)\right)=B_{\epsilon^{\prime \prime}}(0)$. Temos que

(i) a fibra de $\pi$ sobre $\lambda \in B_{\epsilon^{\prime \prime}}(0)$ é naturalmente homeomorfa a $D\left(f_{\lambda^{2}+\mu_{0}}\right) \cap$ $P_{\epsilon, \ldots, \epsilon}(0)$. Como $\left|\mu_{0}\right|<\epsilon^{\prime 2}$, as raízes quadradas de $-\mu_{0}$ estão em $B_{\epsilon^{\prime \prime}}(0)$. Assim, se $\lambda \in B_{\epsilon^{\prime \prime}}(0)$ e $\lambda^{2} \neq-\mu_{0}, D\left(f_{\lambda^{2}+\mu_{0}}\right) \cap P_{\epsilon, \ldots, \epsilon}(0)$ é o discriminante de $f_{\lambda^{2}+\mu_{0}}\left(\right.$ pois $\left.\lambda^{2}+\mu_{0} \in B_{\delta}(0)\right)$.

(ii) Se $a$ e $b$ são as raízes quadradas de $-\mu_{0}$, a restrição de $\pi$ a $\pi^{-1}\left(B_{\epsilon^{\prime \prime}}(0) \backslash\{a, b\}\right)$ é um fibrado localmente trivial.

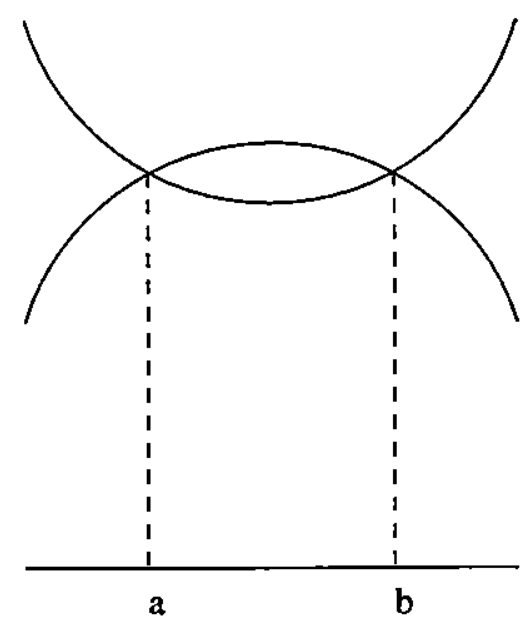

Figura 5.2: Discriminante de uma perturbação estável do bigerme consistindo de duas dobras tangentes

Sejam $A$ e $B$ subconjuntos abertos e contráteis de $B_{\epsilon^{\prime \prime}}(0)$ com interseç̧ão contrátil e não vazia tais que $a \in A \backslash B$ e $b \in B \backslash A$. Como $D\left(g_{\mu_{0}}\right)$ é um fibrado exceto em $\{a, b\}, D\left(g_{\mu_{0}}\right)$ é homotopicamente equivalente a $\pi^{-1}(A \cup B)$. 
Como $A \cap B$ é contrátil e $\pi^{-1}(A \cap B)$ é um fibrado sobre $A \cap B, \pi^{-1}(A \cap B)$ é homotopicamente equivalente à fibra, que é o discriminante de $f_{\lambda}$. Portanto temos dois subespaços, $\pi^{-1}(A)$ e $\pi^{-1}(B)$, cuja intersecção é homotopicamente equivalente ao discriminante de $f_{\lambda}$. Como a suspensão de um espaço pode ser dividida em dois subespaços contráteis cuja interseç̧ão tem vizinhanças tubulares e é homotopicamente equivalente ao espaço original, pelo Lema 5.4 .1 resta mostrar que $\pi^{-1}(A)$ e $\pi^{-1}(B)$ são contráteis. Em $a$, $\gamma(\lambda)=\lambda^{2}+\mu_{0}$ é bianalítica assim, para $\delta^{\prime \prime}>0$ suficientemente pequeno temos um homeomorfismo $\Gamma: \pi^{-1}\left(\gamma^{-1}\left(B_{\delta^{\prime \prime}}(0)\right)\right) \rightarrow D(F) \cap P_{\delta^{\prime \prime}, \epsilon, \ldots, \epsilon}(0)$. Mas $\pi^{-1}(A \backslash\{a\})$ é um fibrado sobre $A \backslash\{a\}$ logo, para $\delta^{\prime \prime}>0$ suficientemente pequeno, $\pi^{-1}(A)$ é homeomorfo a $\pi^{-1}\left(\gamma^{-1}\left(B_{\delta^{\prime \prime}}(0)\right)\right)$ e portanto $\pi^{-1}(A)$ é homeomorfo a $D(F) \cap P_{\delta^{\prime \prime}, \epsilon_{1} \ldots, \epsilon}(0)$. Tomando $\delta^{\prime \prime}<\epsilon$ temos que $D(F) \cap P_{\delta^{\prime \prime}, \epsilon, \ldots, \epsilon}(0)$ é um cone e portanto contrátil. Logo $\pi^{-1}(A)$ é contrátil. Analogamente, $\pi^{-1}(B)$ é contrátil.

Para obter informações sobre a topologia do discriminante de uma perturbação estável de $f$, consideraremos a topologia dos discriminantes de perturbações estáveis das formas normais do Teorema 5.3.20.

Proposiçāo 5.4.3 Seja $h$ um multigerme primitivo de $\mathbb{C}^{n}$ em $\mathbb{C}^{p}$ dado pela forma normal do Teorema 5.3.20 onde $f$ e g são do tipo (2). Então o discriminante de uma perturbação estável de $h$ é homotopicamente equivalente a uma 0-esfera.

Prova Temos que $a=b=0$ e $f\left(x_{1}, \ldots, x_{n}\right)=g\left(x_{1}, \ldots, x_{n}\right)=\sum x_{i}^{2}$. Uma perturbação estável $h_{\lambda}$ de $h$ é dada por (ver Proposição 5.3.7)

$$
h_{\lambda}:\left\{\begin{array}{l}
\left(x_{1}, \ldots, x_{n}\right) \mapsto \sum x_{i}^{2} \\
\left(x_{1}, \ldots, x_{n}\right) \mapsto \sum x_{i}^{2}+\lambda
\end{array}\right.
$$


O discriminante de $h_{\lambda}$ é a união disjunta de dois pontos e portanto é homotopicamente equivalente a uma 0 -esfera.

Proposição 5.4.4 Seja $h$ um multigerme primitivo de $\mathbb{C}^{n}$ em $\mathbb{C}^{p}$ dado pela forma normal do Teorema 5.3.20 onde $f$ é do tipo (3) e g é do tipo (2). Então o discriminante de uma perturbação estável de $h$ tem o tipo de homotopia da suspensão do discriminante de uma perturbação estável de $\tilde{f}_{0}$.

Prova Temos que $a=0$ e $f$ é um desdobramento versal de $\tilde{f}_{0}$. Assim, $h$ é dado por

$$
h:\left\{\begin{aligned}
&\left(x_{1}, \ldots, x_{n}\right) \mapsto\left(\tilde{f}_{x_{n}}\left(x_{1}, \ldots, \dot{x}_{n-1}\right), x_{n}\right) \\
&\left(x_{1}, \ldots, x_{n}\right) \mapsto\left(x_{1}, \ldots, x_{b}, \sum_{i=b+1}^{n} x_{i}^{2}\right)
\end{aligned}\right.
$$

Pela Proposição 5.3.7 existe uma perturbação estável $h_{\lambda}$ de $h$ cujos ramos são $f$ e $g_{\lambda}=g+(0, \lambda)$. O discriminante de $h_{\lambda}$ é a união de dois espaços contráteis: o discriminante de $f$ e o discriminante de $g_{\lambda}$. A intersecção destes conjuntos é o discriminante de $\tilde{f}_{\lambda}$, que é uma perturbação estável de $\tilde{f}_{0}$.

A proposição segue análoga ao Teorema 5.4.2.

Para tratar do caso $f$ e $g$ do tipo (3) precisamos de alguns resultados topológicos.

Sejam $X$ e $Y$ espaços topológicos. O join de $X$ e $Y$, denotado por $X * Y$, é o espaço $(X \times Y \times I) / \sim$ onde $(x, y, \lambda) \sim\left(x^{\prime}, y^{\prime}, \lambda^{\prime}\right)$ se, e somente se, $\lambda=\lambda^{\prime}=0$ e $y=y^{\prime}$ ou $\lambda=\lambda^{\prime}=1$ e $x=x^{\prime}$, ver [5] e [32].

Lema 5.4.5 ([10],Lema 8.5) Se $X_{1}$ é homotopicamente equivalente a $X_{2}$ e $Y_{1}$ é homotopicamente equivalente a $Y_{2}$, então $X_{1} * Y_{1}$ é homotopicamente equivalente a $X_{2} * Y_{2}$. 
Corolário 5.4.6 Se $X_{1}$ é homotopicamente equivalente a $X_{2}$, então $S\left(X_{1}\right)$ é homotopicamente equivalente a $S\left(X_{2}\right)$.

Proposição 5.4.7 Seja $h$ um multigerme primitivo de $\mathbb{C}^{n}$ em $\mathbb{C}^{p}$ dado pela forma normal do Teorema 5.3.20 onde $f$ e $g$ são do tipo (3), ou seja,

$$
h:\left\{\begin{array}{l}
f(\lambda, x, \mu)=\left(\lambda, \tilde{f}_{\mu}(x), \mu\right) \\
g(z, \delta, \mu)=\left(\bar{g}_{\mu}(z), \delta, \mu\right)
\end{array}\right.
$$

Então o discriminante de uma perturbação estável de h é homotopicamente equivalente à suspensão de $D\left(\tilde{f}_{\mu}\right) * D\left(\tilde{g_{\mu}}\right)$.

Prova Temos que $\bar{F}: \mathbb{C}^{n-a-1} \times \mathbb{C} \rightarrow \mathbb{C}^{b} \times \mathbb{C}, \tilde{F}(x, \mu)=\left(\tilde{f}_{\mu}(x), \mu\right)$, e $\tilde{G}:$ $\mathbb{C}^{n-b-1} \times \mathbb{C} \rightarrow \mathbb{C}^{a} \times \mathbb{C}, \tilde{G}(z, \mu)=\left(\tilde{g_{\mu}}(z), \mu\right)$, são desdobramentos miniversais de $\tilde{f}=\tilde{f}_{0}$ e $\tilde{g}=\tilde{g}_{0}$ respectivamente. Segue da Proposição 5.3.7 que um desdobramento miniversal $H$ de $h$ é dado por

$$
H:\left\{\begin{array}{l}
(\nu, \lambda, x, \mu) \mapsto\left(\nu, \lambda, \tilde{f}_{\mu}(x), \mu\right) \\
(\nu, z, \delta, \mu) \mapsto\left(\nu, \tilde{g}_{\mu}(z), \delta, \mu+\nu\right)
\end{array}\right.
$$

Seja $\epsilon>0$ um pseudo raio de Milnor para $\tilde{f}, \tilde{g}$ e $h$. Sejam $P_{\tilde{f}}=P_{\epsilon, \ldots, \epsilon}(0) \subseteq$ $\mathbb{C}^{b}$ e $P_{\tilde{g}}=P_{\epsilon, \ldots, \epsilon}(0) \subseteq \mathbb{C}^{a}$. Consideremos $\delta>0$ um limite de perturbação para o desdobramento $\tilde{F}$ de $\tilde{f}$ com respeito a $P_{\tilde{f}}$ e também para o desdobramento $\tilde{G}$ de $\tilde{g}$ com respeito a $P_{\bar{g}}$. Sejam $\epsilon^{\prime}=\min \{\epsilon, \delta / 2\}$ e $P_{h}=P_{\tilde{g}} \times P_{\tilde{f}} \times B_{\epsilon^{\prime}} \subseteq$ $\mathbb{C}^{a} \times \mathbb{C}^{b} \times \mathbb{C}$. Seja $\delta^{\prime}>0$ um limite de perturbação para o desdobramento $H$ de $h$ com respeito a $P_{h}$. Consideremos $\delta^{\prime \prime}=\min \left\{\delta^{\prime}, \delta / 2\right\}$ e $\nu_{0} \in B_{\delta^{\prime \prime}} \backslash\{0\} \subseteq \mathbb{C}$. Para $\mu \in \mathbb{C}$ definimos $g_{\mu}^{\prime}=\bar{g}_{\mu-\nu_{0}}$ e $G^{\prime}=g_{\mu}^{\prime} \times i d_{\mathbb{C}}$. Seja $h^{\prime}$ dado por

$$
h^{\prime}:\left\{\begin{array}{l}
f(\lambda, x, \mu)=\left(\lambda, \tilde{f}_{\mu}(x), \mu\right) \\
g^{\prime}(z, \delta, \mu)=\left(g_{\mu}^{\prime}(z), \delta, \mu\right)
\end{array}\right.
$$

Temos que $D\left(h_{\nu_{0}}\right) \cap P_{h}=D\left(h^{\prime}\right) \cap P_{h}=\left(D(f) \cap P_{h}\right) \cup\left(D\left(g^{\prime}\right) \cap P_{h}\right)$. Como $D(f) \cap P_{h}$ e $D\left(g^{\prime}\right) \cap P_{h}$ são cones, logo contráteis, segue do Lema 5.4.1 que 
$D\left(h_{\nu_{o}}\right) \cap P_{h}$ é homotopicamente equivalente à suspensão de $D=D(f) \cap$ $D\left(g^{\prime}\right) \cap P_{h}$.

Para terminar a prova é suficiente mostrar que $D$ é homotopicamente equivalente a $\left(D\left(\tilde{f}_{\mu}\right) \cap P_{\tilde{f}}\right) *\left(D\left(\tilde{g}_{\mu}\right) \cap P_{\tilde{g}}\right)$. Denotaremos por $D_{\bar{f}}=D\left(\tilde{f}_{\mu}\right) \cap P_{\tilde{f}}$ e $D_{\bar{g}}=D\left(\tilde{g_{\mu}}\right) \cap P_{\bar{g}}$.

A projeção $\pi_{h}: \mathbb{C}^{a} \times \mathbb{C}^{b} \times \mathbb{C} \rightarrow \mathbb{C}$ induz uma fibração localmente trivial

$$
D \cap \pi_{h}^{-1}\left(B_{\delta^{\prime \prime}} \backslash\left\{0, \nu_{0}\right\}\right) \rightarrow B_{\delta^{\prime \prime}} \backslash\left\{0, \nu_{0}\right\}
$$

cujas fibras são homotopicamente equivalentes a $D_{\tilde{f}} \times D_{\bar{g}}$. Note que $\pi_{h}^{-1}(0)$ é homotopicamente equivalente a $D_{\tilde{g}}$ (pois $D\left(\tilde{f}_{0}\right)$ é contrátil) e $\pi_{h}^{-1}\left(\nu_{0}\right)$ é homotopicamente equivalente a $D_{\bar{f}}$. Observe que isto também ocorre com o join, ou seja, se considerarmos a projeção de $D_{\bar{f}} * D_{\bar{g}}=\left(D_{\bar{f}} \times D_{\bar{g}} \times I\right) / \sim \mathrm{em}$ $I$, as fibras são $D_{\bar{g}}, D_{\tilde{f}}$ e $D_{\bar{f}} \times D_{\bar{g}}$.

Sejam $A$ e $B$ subconjuntos abertos contráteis de $B_{\delta^{\prime \prime}} \subseteq \mathbb{C}$ com interseç̧ão contrátil não vazia tais que $0 \in A \backslash B$ e $\nu_{0} \in B \backslash A$. Analogamente ao Teorema 5.4.2 $D$ é homotopicamente equivalente a $D \cap \pi_{h}^{-1}(A \cup B)$. A seguir, construiremos uma equivalência de homotopia entre $D \cap \pi_{h}^{-1}(A \cup B)$ e $D_{\bar{f}} * D_{\bar{g}}$.

Como $D\left(g_{\mu}^{\prime}\right) \cap P_{\bar{g}}=D\left(G^{\prime}\right) \cap\left(P_{\bar{g}} \times\{\mu\}\right)$ e $A$ é contrátil, existe um homeomorfismo $\alpha: D_{\bar{g}} \times A \rightarrow D\left(G^{\prime}\right) \cap\left(P_{\bar{g}} \times A\right)$ da forma $\alpha_{\mu} \times i d_{A}$. Analogamente existe um homeomorfismo $\beta: D_{\bar{f}} \times B \rightarrow D(\tilde{F}) \cap\left(P_{\bar{f}} \times B\right)$ da forma $\beta_{\mu} \times i d_{B}$.

(i) Construimos primeiramente uma equivalência de homotopia entre $D \cap$ $\left(\pi_{h}^{-1}(A \cap B)\right) \subset \mathbb{C}^{a} \times \mathbb{C}^{b} \times \mathbb{C}$ e $D_{\tilde{f}} \times D_{\tilde{g}} \times\left(\frac{1}{3}, \frac{2}{3}\right) \subset D_{\bar{f}} * D_{\bar{g}}$. Para isto definimos

$$
\begin{aligned}
\phi: D \cap\left(\pi_{h}^{-1}(A \cap B)\right) & \rightarrow D_{\bar{f}} \times D_{\bar{g}} \times\left(\frac{1}{3}, \frac{2}{3}\right) \\
(X, Y, \mu) & \mapsto\left(\beta_{\mu}^{-1}(Y), \alpha_{\mu}^{-1}(X), 1 / 2\right)
\end{aligned}
$$

Dado $c \in A \cap B$ definimos

$$
\begin{aligned}
\psi: D_{\bar{f}} \times D_{\bar{g}} \times\left(\frac{1}{3}, \frac{2}{3}\right) & \rightarrow D \cap\left(\pi_{h}^{-1}(A \cap B)\right) \\
(X, Y, \mu) & \mapsto\left(\alpha_{c}(Y), \beta_{c}(X), c\right)
\end{aligned}
$$


Então

$$
\begin{aligned}
\psi \circ \phi: D \cap\left(\pi_{h}^{-1}(A \cap B)\right) & \rightarrow D \cap\left(\pi_{h}^{-1}(A \cap B)\right) \\
(X, Y, \mu) & \mapsto\left(\left(\alpha_{c} \circ \alpha_{\mu}^{-1}\right)(X),\left(\beta_{c} \circ \beta_{\mu}^{-1}\right)(Y), c\right)
\end{aligned}
$$

Como $A \cap B$ é contrátil temos a homotopia $\Gamma:(A \cap B) \times I \rightarrow A \cap B$ onde $\Gamma(\mu, 0)=\mu$ e $\Gamma(\mu, 1)=c$ para todo $\mu \in A \cap B$. Logo podemos definir uma homotopia entre $\psi \circ \phi$ e a identidade:

$$
\begin{aligned}
\bar{\Gamma}: D \cap\left(\pi_{h}^{-1}(A \cap B)\right) \times I & \rightarrow D \cap\left(\pi_{h}^{-1}(A \cap B)\right) \\
(X, Y, \mu, t) & \mapsto\left(\left(\alpha_{\Gamma(\mu, t)} \circ \alpha_{\mu}^{-1}\right)(X),\left(\beta_{\Gamma(\mu, t)} \circ \beta_{\mu}^{-1}\right)(Y), \Gamma(\mu, t)\right)
\end{aligned}
$$

Para mostrar que $\phi$ é uma equivalência de homotopia resta construir uma homotopia entre $\phi \circ \psi$ e a identidade em $D_{\bar{f}} \times D_{\bar{g}} \times\left(\frac{1}{3}, \frac{2}{3}\right)$. Temos que

$$
\begin{aligned}
\phi \circ \psi: D_{\tilde{f}} \times D_{\tilde{g}} \times\left(\frac{1}{3}, \frac{2}{3}\right) & \rightarrow D_{\tilde{f}} \times D_{\tilde{g}} \times\left(\frac{1}{3}, \frac{2}{3}\right) \\
(X, Y, \mu) & \mapsto(X, Y, 1 / 2)
\end{aligned}
$$

Consideremos a homotopia $\Lambda:\left(\frac{1}{3}, \frac{2}{3}\right) \times I \rightarrow\left(\frac{1}{3}, \frac{2}{3}\right)$ onde $\Lambda(\mu, 0)=\mu \mathrm{e}$ $\Lambda(\mu, 1)=1 / 2$ para todo $\mu \in\left(\frac{1}{3}, \frac{2}{3}\right)$. Logo podemos definir

$$
\begin{aligned}
\vec{\Lambda}: D_{\tilde{f}} \times D_{\tilde{g}} \times\left(\frac{1}{3}, \frac{2}{3}\right) \times I & \rightarrow D_{\bar{f}} \times D_{\tilde{g}} \times\left(\frac{1}{3}, \frac{2}{3}\right) \\
(\bar{X}, \bar{Y}, \mu, t) & \mapsto(\bar{X}, \bar{Y}, \Lambda(\mu, t))
\end{aligned}
$$

que é uma homotopia entre $\phi \circ \psi$ e a identidade.

(ii) Definimos $\left(D_{\bar{f}} \times D_{\bar{g}} \times\left(\frac{1}{3}, 1\right]\right) / \sim \subset D_{\bar{f}} * D_{\bar{g}}$ por $(X, Y, \mu) \sim\left(X^{\prime}, Y^{\prime}, \mu^{\prime}\right)$ se, e somente se, $X=X^{\prime}$ e $\mu=\mu^{\prime}=1$. Estendemos a equivalência de homotopia construida em (i) para uma entre $D \cap \pi_{h}^{-1}(B)$ e $\left(D_{\tilde{f}} \times D_{\tilde{g}} \times\left(\frac{1}{3}, 1\right]\right) / \sim$. Para tal definimos $\left(D_{\bar{g}} \times\left(\frac{1}{3}, 1\right]\right) / \sim$ por $(Y, \mu) \sim\left(Y^{\prime}, \mu^{\prime}\right)$ se, e somente se, $\mu=\mu^{\prime}=1$, e

$$
\begin{aligned}
\varphi: D\left(G^{\prime}\right) \cap\left(P_{\tilde{g}} \times(A \cap B)\right) & \rightarrow D_{\tilde{g}} \times\left(\frac{1}{3}, \frac{2}{3}\right) \\
(X, \mu) & \mapsto\left(\alpha_{\mu}^{-1}(X), 1 / 2\right)
\end{aligned}
$$


Como $\left(D_{\bar{g}} \times\left(\frac{1}{3}, 1\right]\right) / \sim$ é contrátil podemos estender $\varphi$ a uma aplicação $\varphi_{B}$ : $D(\tilde{G}) \cap\left(P_{g} \times B\right) \rightarrow\left(D_{\tilde{g}} \times\left(\frac{1}{3}, 1\right]\right) \sim$. Logo podemos definir

$$
\begin{aligned}
\phi_{B}: D \cap \pi_{h}^{-1}(B) & \rightarrow\left(D_{\tilde{f}} \times D_{\tilde{g}} \times\left(\frac{1}{3}, 1\right]\right) / \sim \\
(X, Y, \mu) & \mapsto\left(\beta_{\mu}^{-1}(Y), \varphi_{B}(Y, \mu)\right)
\end{aligned}
$$

pois podemos identificar $\left(D_{\bar{f}} \times D_{\bar{g}} \times\left(\frac{1}{3}, 1\right]\right) / \sim \operatorname{com} D_{\tilde{f}} \times\left[\left(D_{\bar{g}} \times\left(\frac{1}{3}, 1\right]\right) / \sim\right]$. Note que $\phi_{B}$ estende $\phi$. Analogamente podemos estender $\psi$ a uma aplicação $\psi_{B}:\left(D_{\tilde{f}} \times D_{\tilde{g}} \times\left(\frac{1}{3}, 1\right]\right) / \sim \rightarrow D \cap \pi_{h}^{-1}(B)$. E também analogamente temos que $\phi_{B}$ é uma equivalência de homotopia.

(iii) Analogamente a (ii) estendemos a equivalência de homotopia $\phi$ construida em (i) para $\phi_{A}$ entre $D \cap \pi_{h}^{-1}(A)$ e $\left(D_{\bar{f}} \times D_{\bar{g}} \times\left[0, \frac{2}{3}\right)\right) / \sim$.

Colando $\phi_{A}$ e $\phi_{B}$ obtemos a equivalência de homotopia procurada. $\mathrm{O}$ resultado segue do Corolário 5.4.6.

Lema 5.4.8 Se j e $k$ são números naturais então

(i) $S^{j} * S^{k}$ é homeomorfo a $S^{j+k+1}$.

(ii) A suspensão de $S^{k}$ é homeomorfa a $S^{k+1}$.

Prova Para (i) ver [5] e [32]. O ítem (ii) é uma consequência de (i).

Teorema 5.4.9 Seja $h:\left(\mathbb{C}^{n}, T\right) \rightarrow\left(\mathbb{C}^{p}, 0\right)(n \geq p,(n, p)$ boas dimensões) um multigerme de $\mathcal{A}_{e}$-codimensão 1 e coposto 1 . Então h é quasihomogêneo.

Prova Podemos supor $h$ primitivo.

A prova é por indução sobre o número de ramos de $h$.

Se $|T|=1, h$ é quasihomogêneo por [25][Theorem 1.4]. 
Suponhamos que $h$ tem mais que um ramo. Sejam $f:\left(\mathbb{C}^{n}, S\right) \rightarrow\left(\mathbb{C}^{p}, 0\right)$ e $g:\left(\mathbb{C}^{n}, \tilde{S}\right) \rightarrow\left(\mathbb{C}^{p}, 0\right)$ multigermes tais que $S \cup \tilde{S}=T$ e os ramos de $h$ são os de $f$ e $g$. Por hipótese de indução, se $f$ é transversal ao estrato analítico de $g$, então o pullback de $f$ por este estrato analítico é quasihomogêneo (e analogamente trocando $f$ por $g$ ). Podemos, portanto, aplicar o Teorema 5.3.20. Segue do Teorema 5.3.22 que $h$ é quasihomogêneo.

Corolário 5.4.10 Seja $h$ como no Teorema 5.4.9. Então $\mu_{\Delta}(h)=1$.

Prova Segue de [13] que

$$
\mu_{\Delta}(h) \geq \mathcal{A}_{e}-\operatorname{codim}(h)
$$

e a igualdade ocorre quando $h$ é quasihomogêneo. Logo, o resultado é uma consequência do teorema acima.

Consideremos agora o caso real. Seja $h:\left(\mathbb{R}^{n}, T\right) \rightarrow\left(\mathbb{R}^{p}, 0\right)(n \geq p,(n, p)$ boas dimensões) um multigerme de $\mathcal{A}_{e}$-codimensão 1 e $H=i d_{\mathbb{R}} \times h_{\lambda}$ um desdobramento miniversal. Existem duas escolhas (possivelmente equivalentes) para o discriminante de $h_{\lambda}$ : uma para $\lambda$ positivo e outra para $\lambda$ negativo. Denotaremos por $D^{+}\left(h_{\lambda}\right)$ e $D^{-}\left(h_{\lambda}\right)$ respectivamente. Recordamos que, no caso real, $h$ tem duas aumentações: $k=A_{H}^{+} h$ e $\tilde{k}=A_{H}^{-} h$ com perturbações estáveis $k_{\mu}=i d_{\mathbb{R}} \times h_{\lambda^{2}+\mu}$ e $\tilde{k}_{\mu}=i d_{\mathbb{R}} \times h_{-\lambda^{2}+\mu}$ respectivamente.

Proposição 5.4.11 Com a notação acima

$$
\begin{array}{ll}
(i) D^{+}\left(k_{\mu}\right) \cong D^{+}\left(h_{\lambda}\right) & (i i) D^{-}\left(k_{\mu}\right) \cong S\left(D^{-}\left(h_{\lambda}\right)\right) \\
(i i i) D^{+}\left(\tilde{k}_{\mu}\right) \cong S\left(D^{+}\left(h_{\lambda}\right)\right) & (i v) D^{-}\left(\tilde{k}_{\mu}\right) \cong D^{-}\left(h_{\lambda}\right)
\end{array}
$$


Prova Por simetria é suficiente mostrar a primeira das duas equivalências de homotopia. O caso (ii) é análogo a Proposição 5.4.2 mas se seguirmos a mesma prova em (i), como $-\mu_{0}$ não tem raíz quadrada real, então $D^{+}\left(k_{\mu}\right)$ é um fibrado sobre $B_{\epsilon^{\prime \prime}}(0)$ com fibra $D^{+}\left(h_{\lambda}\right)$. Mas o espaço total de um fibrado sobre um espaço contrátil é homotopicamente equivalente à fibra.

Gostaríamos de obter informações sobre a topologia do discriminante de uma perturbação estável das formas normais do Teorema 5.3.20 no caso real (ver Observaçāo 5.3.23). Resulta que a Proposição 5.4.3 vale no caso real. Também a Proposição 5.4.4 vale numa versão ligeiramente diferente. Aqui temos de considerar os dois discriminantes da perturbação estável de $h$ e os dois discriminantes da perturbação estável de $\tilde{f}_{0}$. Finalmente, se $h$ é um multigerme real nas formas normais do Teorema 5.3.20 onde $f$ e $g$ são do tipo (3), então dados $\tilde{f}$ e $\tilde{g}$, existem duas possibilidades para $h$ : uma dada pela fórmula do Teorema 5.3.20 e a outra substituíndo $f$ nesta fórmula por $(\lambda, x, \mu) \mapsto\left(\lambda, \tilde{f}_{-\mu}(x), \mu\right)$ (ver Observação 5.3.23). Temos o seguinte resultado análogo d Proposiçāo 5.4.7.

Proposição 5.4.12 Seja h como acima. Então os possiveis discriminantes de perturbações estáveis de $h$ são:

$$
\begin{array}{ll}
S\left(D^{+}\left(\tilde{f}_{\mu}\right) * D^{+}\left(\tilde{g}_{\mu}\right)\right) & S\left(D^{-}\left(\tilde{f}_{\mu}\right) * D^{+}\left(\tilde{g}_{\mu}\right)\right) \\
S\left(D^{+}\left(\tilde{f}_{\mu}\right) * D^{-}\left(\tilde{g}_{\mu}\right)\right) & S\left(D^{-}\left(\tilde{f}_{\mu}\right) * D^{-}\left(\tilde{g}_{\mu}\right)\right)
\end{array}
$$

Definição 5.4.13 Seja $g:\left(\mathbb{R}^{n}, S\right) \rightarrow\left(\mathbb{R}^{p}, 0\right)$ um germe de uma aplicação analítica real de $\mathcal{A}$-codimensão finita. Suponhamos que a complexificação 
$g_{\mathbb{C}, t}$ da perturbação real $g_{t}$ de $g$ defina, no domínio $U \subseteq \mathbb{C}^{n}$, uma perturbação estável da complexificação $g_{\mathbb{C}}$ de $g$. Dizemos que $g_{t}$ é uma boa perturbação real se $\operatorname{rank} H_{p-1}\left(D\left(g_{t}\right) ; \mathbb{Z}\right)=\operatorname{rank} H_{p-1}\left(D\left(g_{\mathbb{C}_{t} t}\right) ; \mathbb{Z}\right)$.

Teorema 5.4.14 Seja $h:\left(\mathbb{C}^{n}, T\right) \rightarrow\left(\mathbb{C}^{p}, 0\right)(n \geq p,(n, p)$ boas dimensões $)$ um multigerme de $\mathcal{A}_{e}$-codimensão 1 e coposto 1 . Então existe uma forma real com uma boa perturbação real.

Prova Podemos supor $h$ primitivo. De fato, se $h$ possui uma forma real com uma boa perturbação real, então $D^{+}\left(h_{\lambda}\right)$ ou $D^{-}\left(h_{\lambda}\right)$ tem o tipo de homotopia de uma esfera de dimensão $p-1$. Segue da Proposiçāo 5.4.11 que para uma de suas aumentações reais $k$ ou $\tilde{k}, D^{-}\left(k_{\mu}\right)$ ou $D^{+}\left(\bar{k}_{\mu}\right)$ tem o tipo de homotopia de uma esfera de dimensão $p$. Mas segue do Teorema 5.4.2 que $D\left(k_{\mathbb{C}, \lambda}\right)=S\left(D\left(h_{\mathbb{C}, \lambda}\right)\right)$. Logo $k$ tem uma forma real com uma boa perturbação real.

A prova é por induçăo sobre o número de ramos de $h$, analogamente à prova do Teorema 5.4.9.

$O$ resultado para monogerme segue de [25].

Suponhamos que $h$ tenha mais que um ramo. Como $h$ é quasihomogêneo, podemos aplicar o Teorema 5.3.20, que consiste na classificação de multigermes primitivos. Temos então de analisar as três possiveis formas normais de $h$ dadas por este teorema. Sejam $f$ e $g$ multigermes tais que os ramos de $h$ são os de $f$ e $g$. Se $f$ e $g$ são do tipo (2), o resultado segue da Proposição 5.4.3 que como vimos vale no caso real. Se $f$ é do tipo (3) e $g$ é do tipo (2), o resultado segue da Proposição 5.4 .4 como acima. Finalmente, se $f$ e $g$ são do tipo (3), o resultado segue da Proposição 5.4.7 e Proposição 5.4.12. 


\section{Capítulo 6}

\section{Multigermes de $\mathcal{A}_{e}$-codimensão arbitrária}

\subsection{Introdução}

No Capítulo 5 definimos aumentação para um multigerme de $\mathcal{A}_{e}$-codimensão 1. Um dos resultados mais interessantes daquele capítulo é o Teorema 5.4.2 que diz que o discriminante de uma perturbação estável da aumentação é homotopicamente equivalente à suspensão do discriminante de uma perturbação estável do germe inicial.

Em [17] K.Houston generaliza a definição de aumentação para multigermes de codimensão arbitrária e dá uma fórmula relacionando as codimensōes. $\mathrm{Na}$ secção 6.2 descrevemos estes resultados.

Para esta definição mais geral de aumentação obtemos um resultado que é uma generalização do Teorema 5.4.2, que será descrito na secção 6.3. Mostraremos que é possivel descrever a topologia do discriminante de uma perturbação estável da aumentação em termos da topologia do discriminante de 
uma perturbação estável do germe.

\subsection{Resultados gerais}

Definição 6.2.1 Seja $f:\left(\mathbb{C}^{n}, S\right) \rightarrow\left(\mathbb{C}^{p}, 0\right)$ um multigerme onde $S$ é um subconjunto finito de $\mathbb{C}^{n}$. Suponhamos que exista um desdobramento a um parâmetro

$$
\begin{aligned}
F:\left(\mathbb{C} \times \mathbb{C}^{n},\{0\} \times S\right) & \rightarrow\left(\mathbb{C} \times \mathbb{C}^{p},(0,0)\right) \\
(\lambda, x) & \mapsto(\lambda, \tilde{F}(\lambda, x))
\end{aligned}
$$

de f. Se $g:\left(\mathbb{C}^{q}, 0\right) \rightarrow(\mathbb{C}, 0)$ é o germe de uma função holomorfa, definimos

$$
\begin{aligned}
A_{F, g} f:\left(\mathbb{C}^{n} \times \mathbb{C}^{q}, S \times\{0\}\right) & \rightarrow\left(\mathbb{C}^{p} \times \mathbb{C}^{q},(0,0)\right) \\
(x, z) & =(\tilde{F}(g(z), x), z)
\end{aligned}
$$

$A_{F, g} f$ é dita aumentaçāo de $f$ por $F$ e g. Também dizemos que $f$ foi aumentada por $F$ e $g$.

No Capítulo 5 estudamos um tipo especial de aumentação, ou seja, quando $f$ tem $\mathcal{A}_{e}$-codimensāo $1, F$ é miniversal e $g(z)=z^{2}$.

Exemplo 6.2.2 A série $S_{k}:\left(\mathbb{C}^{2}, 0\right) \rightarrow\left(\mathbb{C}^{3}, 0\right)$ dada por

$$
(x, y) \mapsto\left(x, y^{2}, y^{3}+x^{k+1} y\right)
$$

pode ser obtida da série $A_{k}:(\mathbb{C}, 0) \rightarrow(\mathbb{C}, 0), A_{k}(z)=z^{k+1}$, por aumentação. De fato, consideremos $f:(\mathbb{C}, 0) \rightarrow\left(\mathbb{C}^{2}, 0\right)$ dada por $f(x)=\left(x^{2}, x^{3}\right)$. Um desdobramento a 1-parâmetro de $f$ é dado por

$$
\begin{aligned}
F:(\mathbb{C} \times \mathbb{C}, 0) & \rightarrow\left(\mathbb{C} \times \mathbb{C}^{2}, 0\right) \\
(\lambda, x) & \mapsto\left(\lambda, x^{2}, x^{3}+\lambda x\right)
\end{aligned}
$$


(de fato $F$ é miniversal). Se $g:(\mathbb{C}, 0) \rightarrow(\mathbb{C}, 0)$ é dada por $g(z)=z^{k+1}$ então $A_{F, g}(f):(\mathbb{C} \times \mathbb{C}, 0) \rightarrow\left(\mathbb{C}^{2} \times \mathbb{C}, 0\right)$ é dada por

$$
A_{F, g}(f)(x, z)=\left(x^{2}, x^{3}+z^{k+1} x, z\right)
$$

que é equivalente a $S_{k}$.

Exemplo 6.2.3 Seja $f:(\mathbb{C}, 0) \rightarrow\left(\mathbb{C}^{2}, 0\right), f(x)=\left(x^{2}, x^{2 k+1}\right)$. Temos que $\mathcal{A}_{e}$-codimensão $(f)=k$. Um desdobramento a 1-parâmetro de $f$ é dado por $F:\left(\mathbb{C}^{2}, 0\right) \rightarrow\left(\mathbb{C}^{3}, 0\right), F(\lambda, x)=\left(\lambda, x^{2}, x^{2 k+1}+\lambda x\right)$. Se $g:(\mathbb{C}, 0) \rightarrow$ $(\mathbb{C}, 0)$ é dada por $g(z)=z^{2}$ então $A_{F, g}(f)(x, z)=\left(x^{2}, x^{2 k+1}+z^{2} x, z\right)$, que é equivalente à série $B_{k}$ (ver Teorema 2.4.6).

Proposição 6.2.4 ([17], Teorema 3.3) Suponhamos que o desdobramento $F$ de $f$ seja estável como germe. Então

$$
\mathcal{A}_{e}-\operatorname{codim}(f) \cdot \tau(g) \leq \mathcal{A}_{e}-\operatorname{codim}\left(A_{F, g}(f)\right)
$$

onde $\tau(g)=\operatorname{dim}_{\mathbb{C}} \frac{\mathcal{O}_{q}}{\left\langle g, \frac{\partial g}{\partial z_{1}}, \ldots, \frac{\partial g}{\partial z_{q}}\right\rangle}$ é o número de Tjurina de $g$. Além disso, se $F$ ou $g$ é quasihomogêneo entâo

$$
\mathcal{A}_{e}-\operatorname{codim}(f) \cdot \tau(g)=\mathcal{A}_{e}-\operatorname{codim}\left(A_{F, g}(f)\right)
$$

\subsection{Topologia da aumentação}

Vimos na secção 5.4 que para $n \geq p,(n, p)$ no domínio das boas dimensões segundo Mather e $f$ finitamente determinado, $\mu_{\Delta}(f)$ satisfaz

$$
\mu_{\Delta}(f) \geq \mathcal{A}_{e}-\operatorname{codim}(f)
$$


e a igualdade ocorre quando $f$ é quasihomogêneo. Se $g$ é quasihomogêneo, segue da Proposição 6.2.4 que

$$
\mu_{\Delta}\left(A_{F, g}(f)\right)=\mathcal{A}_{e}-\operatorname{codim}\left(A_{F, g}(f)\right)=\mathcal{A}_{e}-\operatorname{codim}(f) \cdot \tau(g)=\mu_{\Delta}(f) \cdot \mu(g)
$$

Portanto o discriminante de uma perturbação estável de $A_{F, g}(f)$ é homotopicamente equivalente ao join da fibra de Milnor de $g$ e do discriminante de uma perturbação estável de $f$.

Nosso objetivo é mostrar que este resultado vale mais geralmente.

Seja $G:\left(\mathbb{C} \times \mathbb{C}^{n} \times \mathbb{C}^{q},\{0\} \times S \times\{0\}\right) \rightarrow\left(\mathbb{C} \times \mathbb{C}^{p} \times \mathbb{C}^{q}, 0\right)$ dada por

$$
G(\mu, x, z)=(\mu, \tilde{F}(g(z)+\mu, x), z)
$$

Então $G$ é um desdobramento a 1-parâmetro de $A_{F, g}(f)$.

Lema 6.3.1 Se F é estável como germe então $G$ também é estável.

Prova Seja $\varphi:\left(\mathbb{C} \times \mathbb{C}^{n} \times \mathbb{C}^{q},\{0\} \times S \times\{0\}\right) \rightarrow\left(\mathbb{C} \times \mathbb{C}^{n} \times \mathbb{C}^{q},\{0\} \times S \times\{0\}\right)$ dada por

$$
\varphi(\mu, x, z)=(\mu+g(z), x, z)
$$

e $\psi:\left(\mathbb{C} \times \mathbb{C}^{p} \times \mathbb{C}^{q}, 0\right) \rightarrow\left(\mathbb{C} \times \mathbb{C}^{p} \times \mathbb{C}^{q}, 0\right)$ dada por

$$
\psi(\mu, y, z)=(\mu-g(z), y, z)
$$

Temos que $\varphi$ e $\psi$ são difeomorfismos e

$$
G=\psi \circ\left(F \times i d_{C}\right) \circ \varphi
$$

ou seja, $G$ é $\mathcal{A}$-equivalente (como germe) a $F \times i d_{\mathbb{C}}$. Logo $G$ é estável.

Lema 6.3.2 $D(G)=\psi\left(D\left(F \times i d_{\mathbb{C}^{q}}\right)\right)$. 
Prova $G$ é submersão se, e somente se, $F \times i d_{\mathbb{C}}$ é submersão.

Teorema 6.3.3 Suponhamos que o desdobramento $F$ de $f$ seja estável. Então o discriminante de uma perturbação estável de $A=A_{F, g}(f)$ é homotopicamente equivalente ao join do discriminante de uma perturbação estável de $f$ e da fibra de Milnor de $g$.

Prova Seja $f_{\lambda}(x)=\tilde{F}(\lambda, x)$ e $A_{\mu}(x, z)=(\bar{F}(\mu+g(z), x), z)$. Como $D(G)=\psi\left(D\left(F \times i d_{\mathbb{C}}\right)\right)$ e $D\left(f_{\lambda}\right)=D(F) \cap\left(\{\lambda\} \times \mathbb{C}^{p}\right)$ temos que

$$
\begin{aligned}
D\left(A_{\mu_{0}}\right) & =D(G) \cap\left(\left\{\mu_{0}\right\} \times \mathbb{C}^{p} \times \mathbb{C}^{q}\right) \\
& =\psi\left(D\left(F \times i d_{\mathbb{C}^{q}}\right)\right) \cap\left(\left\{\mu_{0}\right\} \times \mathbb{C}^{p} \times \mathbb{C}^{q}\right) \\
& =\left\{(\mu-g(z), y, z) /(\mu, y) \in D(F) e(\mu-g(z), y, z) \in\left\{\mu_{0}\right\} \times \mathbb{C}^{p} \times \mathbb{C}^{q}\right\} \\
& =\left\{\left(\mu_{0}, y, z\right) /\left(\mu_{0}+g(z), y\right) \in D(F)\right\} \\
& =\left\{\left(\mu_{0}, y, z\right) / y \in D\left(f_{\mu_{0}+g(z)}\right)\right\}
\end{aligned}
$$

Consideremos a projeção natural

$$
\pi: D\left(A_{\mu_{0}}\right) \rightarrow \mathbb{C}^{q}
$$

e a composição

$$
D\left(A_{\mu_{0}}\right) \stackrel{\pi}{\longrightarrow} \mathbb{C}^{q} \stackrel{g}{\longrightarrow} \mathbb{C}
$$

Então para $\epsilon>0$ suficientemente pequeno,

$$
D\left(A_{\mu_{0}}\right) \cap(g \circ \pi)^{-1}\left(B_{\epsilon} \backslash\left\{0,-\mu_{0}\right\}\right) \rightarrow B_{\epsilon} \backslash\left\{0,-\mu_{0}\right\}
$$

é uma fibração localmente trivial cujas fibras são homotopicamente equivalentes a $D\left(f_{\lambda}\right) \times g^{-1}(\lambda)$. Segue analogamente à prova do Teorema 5.4.7 que $D\left(A_{\mu_{0}}\right)$ é homotopicamente equivalente a $D\left(f_{\lambda}\right) * g^{-1}(\lambda)$. 
Observação 6.3.4 Suponhamos que $F$ seja um desdobramento estável de $f$. Se o par $(n, p)$ está no domínio das boas dimensões segundo Mather ou $g$ é quasihomogêneo, temos que

$$
\mathcal{A}_{e}-\operatorname{codim}(f) \cdot \tau(g)=\mathcal{A}_{e}-\operatorname{codim}\left(A_{F, g}(f)\right)
$$

Assim, segue como corolário de 6.2.4 que $A_{F, g}(f)$ é finitamente determinado se, e somente se, $g$ define uma singularidade isolada.

Um caso a ser considerado posteriormente é quando $g$ é uma singularidade não isolada. Neste caso, se o tipo de homotopia da fibra de Milnor de $g$ é conhecido, podemos determinar o tipo de homotopia do discriminante de uma perturbação estável de $A_{F, g}(f)$, pois, mesmo no caso $n<p$, o discriminante de uma perturbação estável de $f$ é homotopicamente equivalente a um bouquet de esferas. 


\section{Referências Bibliográficas}

[1] V.I.Arnol'd, Critical points of functions on manifolds with boundary, Russian Math. Surveys 33 (1978), 99-116.

[2] V.I.Arnol'd, Critical points of functions on a manifold with boundary, the simple Lie groups $B_{k}, C_{k}$, and $F_{4}$ and singularities of evolutes, London Math. Soc. Lecture Note Series 53 (1981), 207-224.

[3] V.I.Arnol'd, Dynamical Systems VIII : Singularity Theory II - Applications, Encyclopaedia of Mathematical Sciences 39 (1993), Springer Verlag.

[4] V.I.Arnol'd, Normal forms for functions near degenerate critical points, the Weyl groups of $A_{k}, D_{k}, E_{k}$ and Lagrangian singularities, Functional Anal. Appl. 6 (1973), 254-272.

[5] V.I.Arnol'd, S.M.Gusein-Zade and A.N.Varchenko, Singularities of differentiable maps, Vol. II, Monodromy and asymptotics of integrals, Monographs in Mathematics Vol.83 (1988), Birkhäuser.

[6] R.Atique, T.Cooper and D.Mond, Vanishing topology of codimension 1 multigerms over $\mathbb{R}$ and $\mathbb{C}$, preprint, 1997. 
[7] M.F.Atiyah and I.G.MacDonald, Introduction to commutative algebra, Addison-Wesley Publishing Company (1969).

[8] D.Bayer and M.Stillman, Macaulay, a computer algebra system for algebraic geometry, available by anonymous ftp from zariski.harvard.edu (login: anonymous, password: any, cd Macaulay), 1982-1990.

[9] J.W.Bruce and M.Roberts, Critical points of functions on analytic varieties, Topology 27 (1988), 57-90.

[10] T.Cooper, Mapgerms of $\mathcal{A}_{e}$-codimension one, $\mathrm{Ph}$. D. Thesis, University of Warwick, 1994.

[11] J.Damon, $\mathcal{A}$-equivalence and the equivalence of sections of images and discriminants, Springer Lecture Notes in Mathematics 1462 (1991), 93121.

[12] J.Damon, The unfolding and determinacy theorems for subgroups of $\mathcal{A}$ and $\mathcal{K}$, Singularities Proceedings of Symposia in Pure Mathematics 40:1 (American Mathematical Society, Providence, 1983).

[13] J.Damon and D.Mond, $\mathcal{A}$-codimension and the vanishing topology of discriminants, Invent. math. 106 (1991), 217-242.

[14] C.Gibson, Singular points of smooth mappings, Pitman Research Notes in Mathematics, 25 (1979).

[15] M.Golubitsky and V.Guillemin, Stable mappings and their singularities, Springer-Verlag (1973).

[16] V.V.Goryunov and D.Mond, Vanishing cohomology of singularities of mappings, Compositio Mathematica 89 (1993), 45-80. 
[17] K.Houston, On singularities of folding maps and augmentations, to appear, Math. Scandinavica.

[18] C.Hobbs and N.Kirk, On the classification and bifurcation of multigerms of maps from surfaces to 3-space, preprint, Oxford Brookes University, 1996.

[19] S.Izumiya and S.Matsuoka, Notes on smooth function germs on varieties, Proc. Amer. Math. Soc. 97 (1986), 146-150.

[20] E.J.N.Looijenga, Isolated singular points on complete intersections, London Math. Soc. Lecture Note Series 77.

[21] W.L.Marar, Mapping fibrations, Manuscripta Math. 80 (1993), 273-281.

[22] W.L.Marar and D.Mond, Multiple point schemes for corank 1 maps, Jour. London Math. Soc. (2) 39 (1989), 553-567.

[23] J.N.Mather, Stability of $C^{\infty}{ }_{-}$mappings IV: Classification of stable mapgerms by $\mathcal{R}$-algebras, Publ. Math. IHES 37 (1969), 223-248.

[24] M.Miyanishi, Algebraic Geometry, Translations of Mathematical Monographs (136), A.M.S. (1994).

[25] D.Mond, How good are real pictures?, Progress in Maths. 134, Birkhauser-Verlag (1996), 259-276.

[26] D.Mond, On the classification of germs of maps from $\mathbb{R}^{2}$ to $\mathbb{R}^{3}$, Proc. London Math. Soc. 50 (1985), 333-369.

[27] D.Mond, Singularities of mappings from surfaces to 3-space, Singularity Theory, D.T,Lê, K.Saito, B.Tessier, eds, World Scientific, Singapore (1995), 509-526. 
[28] D.Mond, Vanishing cycles for analytic maps, Springer Lecture Notes in Mathematics 1462 (1991), 221-234.

[29] D.Mond and J.Montaldi, Deformations of maps on complete intersections, Damon's $\mathcal{K}_{V}$-equivalence and bifurcations, London Math. Soc. LNS 201 (1991), Cambridge University Press 263-284.

[30] P.Orlik and H.Terao, Arrangements of Hyperplanes, Springer-Verlag (1991).

[31] R.Pellikaan, Finite determinacy of functions with non-isolated singularities, Proc. London Math. Soc. (3) 57 (1988), 357-382.

[32] D.Rolfsen, Knots and links, Publish or Perish, 1976.

[33] M.A.S.Ruas, $C^{l}$-Determinação finita e aplicações, Tese de Doutorado, ICMSC-USP, São Carlos, 1983.

[34] E.H.Spanier, Algebraic Topology, McGraw-Hill (1966).

[35] C.T.C.Wall, Finite determinacy of smooth map-germs, Bull. London Math. Soc. 13 (1981), 481-539. 